\title{
Involutions of a Clifford algebra induced by involutions of orthogonal group in characteristic 2
}

\author{
M. G. Mahmoudi, A.-H. Nokhodkar
}

September 26, 2012

\begin{abstract}
Among the involutions of a Clifford algebra, those induced by the involutions of the orthogonal group are the most natural ones. In this work, several basic properties of these involutions, such as the relations between their invariants, their occurrences and their decompositions are investigated.
\end{abstract}

Mathematics Subject Classification: 11E88, 11E04, 16W10.

\section{Introduction}

The main object of this work is to study the involutions of a Clifford algebra induced by the involutions of the orthogonal group in characteristic two. For the case of characteristic $\neq 2$, these involutions were studied in [8] in connection with the periodicity of real Clifford algebras with involution. Also they were considered in [15] in connection with the Pfister Factor Conjecture, which was finally settled in [1]. In characteristic $\neq 2$, some properties of these involutions were also investigated in [9].

Our starting point is a general analysis of involutions of orthogonal group in characteristic 2. For the case of characteristic different from 2, the situation is much more straightforward as it is known that if $\tau$ is an involution in the orthogonal group $O(V, q)$ then there exists a decomposition $V=U \perp U^{\perp}$ with $\left.\tau\right|_{U}=$ id and $\left.\tau\right|_{U^{\perp}}=-$ id. For the case where the characteristic of the base field is 2 , the situation is quite more subtle. Wiitala in [17, Thm. 2] provides a characterization of involutions of orthogonal groups in characteristic 2 . For every involution $\tau$ in $O(V, q)$, he proves that there exists a decomposition $V=W \perp V_{1} \perp V_{2} \perp \cdots$, where the restriction of $\tau$ to $W$ is trivial and exactly one of the following is true: (1) each $V_{i}$ is a two-dimensional subspace of $V$ and the restriction of $\tau$ to $V_{i}$ is a reflection; (2) each $V_{i}$ is a four-dimensional subspace of $V$ invariant under $\tau$ such that the fixed space of $\left.\tau\right|_{V_{i}}$ is a totally isotropic space of dimension 2. In $\S 3$, several geometric interpretations of Wiitala's result are obtained and his characterization is complemented.

An initial observation for the study of the involutions is that every involution of a Clifford algebra $C(V)$ which leaves $V$ invariant, is induced by an involution 
in the orthogonal group $O(V, q)$. Let $\tau \in O(V, q)$ be an involution and let $J_{\tau}$ be the involution induced by $\tau$ on $C(V)$. In $\S 4$, elementary investigations about these involutions such as the calculation of their type (that is if $J_{\tau}$ is of orthogonal or symplectic type) are done. Also some relations between invariants of $J_{\tau}$ (e.g., the type and the discriminant (in the sense of [7])) and invariants of $\tau$ (e.g., the Dickson invariant and the spinor norm) are obtained. Finally, in the case of the nontriviality of the Arf invariant, these questions for the restriction of $J_{\tau}$ to the even Clifford algebra $C_{0}(V)$ are investigated.

In $\S 5$, involutorial versions of some relations between the Clifford algebra and the even Clifford algebra (needed in the sequel) obtained by Mammone, Tignol and Wadsworth in [10] are presented. These relations link an orthogonal sum of a quadratic plane (i.e., a two-dimensional quadratic space) over $F$ and an $n$-dimensional quadratic space over $F$ to the Clifford algebra of an $(n+2)$ dimensional quadratic space.

In $\S 6$, analogous to [9], a geometric characterization of an involution of a quaternion algebra is obtained. It turns out that if $(Q, \sigma)$ is a quaternion algebra with involution then there exists a quadratic plane $(\mathbb{E}, \varphi)$ and an involution $\tau \in O(\mathbb{E}, \varphi)$ such that $(Q, \sigma) \simeq\left(C(\mathbb{E}), J_{\tau}\right)$. Also $\sigma$ is of symplectic (resp. orthogonal) type if and only if $\tau=$ id (resp. $\tau$ is a reflection), see (6.1). In view of this result, it is deduced that as an algebra with involution, $(Q, \sigma)$ is characterized by the spinor norm of $\tau$. Then we show that a multiquaternion algebra with involution $(A, \sigma):=\left(Q_{1}, \sigma_{1}\right) \otimes \cdots \otimes\left(Q_{n}, \sigma_{n}\right)$ is isomorphic to the Clifford algebra with involution $\left(C(V), J_{\tau}\right)$ where $\tau \in O(V, q)$ is a suitable involution of a certain quadratic space $(V, q)$. Also a more precise description of $\tau$, depending on the type of $\sigma$, is given. In particular it is shown that if $\sigma$ is of symplectic type then the involution $\tau \in O(V, q)$ can be chosen to be identity and if $\sigma$ is of orthogonal type then $\tau$ can be chosen to be an orthogonal sum of reflections (see (6.3)).

Let $(Q, \sigma)$ be a quaternion algebra with an involution of the second kind. In contrary to what happens in characteristic different from 2 (see $[9,(6.10)]$ ), $(Q, \sigma)$ is not isomorphic to a Clifford algebra with involution $\left(C(V), J_{\tau}\right)$ for any $(V, q)$ and $\tau(6.9)$. Instead of that it is shown that $(Q, \sigma)$ is isomorphic to an even Clifford algebra with involution $\left(C_{0}(V), J_{\tau}\right)$ where $(V, q)$ is a quadratic space of dimension 4 over the fixed field of $\sigma$ restricted to the center of $Q$. More generally if $K / F$ is a separable quadratic extension with nontrivial automorphism $\rho$, it is shown that the multiquaternion algebra $(A, \sigma):=$ $(K, \rho) \otimes\left(Q_{1}, \sigma_{1}\right) \otimes \cdots \otimes\left(Q_{n}, \sigma_{n}\right)$ is isomorphic to the even Clifford algebra with involution $\left(C_{0}(V), J_{\tau}\right)$ for a suitable quadratic space $(V, q)$ over $F$ and an involution $\tau$ in $O(V, q)$. Furthermore it is shown that if $n \equiv 0 \bmod 2$ then $\tau$ can be chosen to be identity and if $n \equiv 1 \bmod 2$ then $\tau$ can be chosen to be a reflection (see $(6.7)$ ). In should be mentioned that in low dimensions (e.g., $n \leqslant 2$ ), these results, for arbitrary characteristic, are already included in [5].

The main object of $\S 7$ is to investigate the behavior of the involution $J_{\tau}$ on $C_{0}(V)$ in the case where $(V, q)$ has trivial Arf invariant. Note that as in this case $C_{0}(V)$ is not central simple, one may wonder how does $J_{\tau}$ act on simple components of $C_{0}(V)$. Also one may want to see what is the relation between the spinor norm of $\tau$ and the discriminant of the restriction of $J_{\tau}$ to simple 
components of $C_{0}(V)$. In this section these questions and some other related questions similar to those investigated in the previous sections, are studied.

It is relevant to mention that in characteristic $\neq 2$, the behavior of $J_{\tau}$ on simple components of $C_{0}(V)$, even for the simplest case $\tau=$ id, has been of importance in many applications of Clifford algebras in the literature (see, e.g., [3], [16], [11], [13]).

In characteristic 2 , when $\tau$ is arbitrary we are able to give a precise description of the action of $J_{\tau}$ on simple components of $C_{0}(V)$. It turns out that, depending on $\tau$, the involution $J_{\tau}$ can also be a switch involution. See (7.8) and (7.11).

In a final appendix, a universal mapping property for the even Clifford algebra, analogous to $[9,(3.3)]$, which is used to shorten some proofs in the paper is presented.

\section{Preliminaries and terminology}

Let $F$ be a field of characteristic 2 and let $V$ be a finite dimensional vector space over $F$. A quadratic form over $F$ is a map $q: V \rightarrow F$ such that $q(\lambda u+$ $\mu v)=\lambda^{2} q(u)+\mu^{2} q(v)+\lambda \mu B(u, v)$ for every $\lambda, \mu \in F$ and $u, v \in V$, where $B: V \times V \rightarrow F$ is a bilinear form over $V$. We have $B(u, v)=q(u)+q(v)+$ $q(u+v)$ and so the bilinear form $B$ is uniquely determined by $q$. In particular $B(v, v)=0$ for every $v \in V$, i.e., $B$ is an alternating form. Two vectors $u, v \in V$ are called orthogonal if $B(u, v)=0$. The radical of $V$ is defined as $\operatorname{rad} V=\{x \in V \mid B(x, y)=0$ for every $y \in V\}$. We say that $q$ is nondefective if $\operatorname{ker}\left(\left.q\right|_{\operatorname{rad} V}\right)=0$. If the bilinear form $B$ is nondegenerate, $(V, q)$ is called regular. We call $(V, q)$ or $(V, q, B)$ a quadratic space if $q$ is regular. Any quadratic space $(V, q)$ is necessarily even dimensional and has a basis $\mathcal{B}=\left\{u_{1}, v_{1}, \cdots, u_{n}, v_{n}\right\}$ such that $B\left(u_{i}, v_{i}\right)=1$ for each $i$ and all other distinct pairs of $\mathcal{B}$ are orthogonal (see [14, p. 339]). Such a basis is called a symplectic basis of $V$. We denote by $[a, b]$ the two dimensional quadratic space $(V, q)$ where $V=F u+F v, q(u)=a$, $q(v)=b$ and $B(u, v)=1$. Also we call $\{u, v\}$ a standard symplectic basis of $(V, q)$.

We say that $q$ represents $a \in F$, if there is a nonzero vector $v \in V$ such that $q(v)=a$. The set of values in $F^{\times}=F \backslash\{0\}$ represented by $q$ is denoted by $D_{F}(q)$. The quadratic space $(V, q)$ is called universal if $D_{F}(q)=F^{\times}$. If $(V, q, B)$ is a quadratic space and $a \in F^{\times}$, the scaled quadratic space $(V, a \cdot q, a \cdot B)$ is defined by $a \cdot q(v)=a q(v)$ for every $v \in V$.

An isometry between two quadratic spaces such as $\left(V_{1}, q_{1}\right) \simeq\left(V_{2}, q_{2}\right)$ is an isomorphism of vector spaces $\tau: V_{1} \rightarrow V_{2}$ such that $q_{2}(\tau(v))=q_{1}(v)$ for every $v \in V_{1}$. Similarly, an isometry between two bilinear spaces such as $\left(V_{1}, B_{1}\right) \simeq\left(V_{2}, B_{2}\right)$ is an isomorphism of vector spaces $\tau: V_{1} \rightarrow V_{2}$ such that $B_{2}(\tau(u), \tau(v))=B_{1}(u, v)$ for every $u, v \in V_{1}$. Note that $\left(V_{1}, q_{1}\right) \simeq\left(V_{2}, q_{2}\right)$ implies that $\left(V_{1}, B_{1}\right) \simeq\left(V_{2}, B_{2}\right)$ but the converse is not always true. The group of isometries of $(V, q)$ is denoted by $O(V, q)$ and is called the orthogonal group of $(V, q)$.

Let $\left(V_{1}, q_{1}\right)$ and $\left(V_{2}, q_{2}\right)$ be two quadratic spaces over $F$ and define the quadratic form $q_{1} \perp q_{2}$ over $V_{1} \oplus V_{2}$ via $\left(q_{1} \perp q_{2}\right)\left(v_{1}+v_{2}\right)=q_{1}\left(v_{1}\right)+q_{2}\left(v_{2}\right)$. We denote this quadratic space by $\left(V_{1} \perp V_{2}, q_{1} \perp q_{2}\right)$ and we call it the orthogonal 
sum of $\left(V_{1}, q_{1}\right)$ and $\left(V_{2}, q_{2}\right)$. If $\left(V_{1}, q_{1}\right)$ and $\left(V_{2}, q_{2}\right)$ are quadratic spaces over $F$ and $\tau_{1}$ and $\tau_{2}$ are isometries of $\left(V_{1}, q_{1}\right)$ and $\left(V_{2}, q_{2}\right)$, respectively, the orthogonal sum of $\tau_{1}$ and $\tau_{2}$ which is denoted by $\tau_{1} \perp \tau_{2}$ is an isometry of $\left(V_{1} \perp V_{2}, q_{1} \perp q_{2}\right)$ defined by $\left(\tau_{1} \perp \tau_{2}\right)\left(v_{1}+v_{2}\right)=\tau_{1}\left(v_{1}\right)+\tau_{2}\left(v_{2}\right)$.

All $F$-algebras considered in this work are implicitly supposed to be associative. The center of an $F$-algebra $A$ is denoted by $Z(A)$ and the group of invertible elements of $A$ is denoted by $A^{*}$. If $u \in A$ is an invertible element, we use the notation $\operatorname{Int}(u)$ for the inner automorphism of $A$ induced by $u$ :

$$
\operatorname{Int}(u): A \rightarrow A, \quad \operatorname{Int}(u)(x)=u x u^{-1} .
$$

Let $(V, q)$ be a quadratic space over a field $F$ of characteristic 2 and let $A$ be an $F$-algebra. A linear map $f: V \rightarrow A$ is called compatible with $q$ if for every $x \in V$, we have $f(x)^{2}=q(x) \cdot 1$. In particular if $\left\{v_{1}, \cdots, v_{n}\right\}$ is a basis of $V$, then the linear map $f$ is compatible with $q$ if and only if for every $1 \leqslant i \leqslant n$, we have $f\left(v_{i}\right)^{2}=q\left(v_{i}\right) \cdot 1$ and for every $1 \leqslant i, j \leqslant n$, we have $f\left(v_{i}\right) f\left(v_{j}\right)+f\left(v_{j}\right) f\left(v_{i}\right)=B\left(v_{i}, v_{j}\right) \cdot 1$.

The Clifford algebra of a quadratic space $(V, q)$ is denoted by $C(V, q)$, or $C(V)$ if no confusion arises, and we consider $V$ as a subspace of $C(V)$. We have $u v=v u+B(u, v) \cdot 1$ for every $u, v \in V$. The even Clifford algebra of $(V, q)$ is denoted by $C_{0}(V, q)$ or $C_{0}(V)$.

If $\tau$ is an isometry of $(V, q)$, then $\tau$ induces an algebra automorphism $I_{\tau}$ of $C_{0}(V)$. The Dickson invariant

$$
\mathrm{D}: O(V, q) \rightarrow \mathbb{Z} / 2 \mathbb{Z}
$$

is defined by $\mathrm{D}(\tau)=0 \in \mathbb{Z} / 2 \mathbb{Z}$, if $\left.I_{\tau}\right|_{Z\left(C_{0}(V)\right)}=$ id and $\mathrm{D}(\tau)=1 \in \mathbb{Z} / 2 \mathbb{Z}$ otherwise.

Consider the additive subgroup $\wp(F)=\left\{x+x^{2} \mid x \in F\right\}$ of $(F,+)$. Let $\mathcal{B}=\left\{u_{1}, v_{1}, \cdots, u_{n}, v_{n}\right\}$ be a symplectic basis of $(V, q)$. It can be shown that the class of $\sum_{i=1}^{n} q\left(u_{i}\right) q\left(v_{i}\right)$ in the quotient group $F / \wp(F)$, which is called the Arf invariant of $(V, q)$ and is denoted by $\Delta(V, q)$ or $\Delta(q)$, is independent of the choice of $\mathcal{B}$ (see $[14$, Ch. 9, (4.2)]). In particular the Arf invariant of the form $[a, b]$ is $a b+\wp(F)$.

If $(V, q)$ is a quadratic space, a nonzero vector $v \in V$ is called isotropic if $q(v)=0$ and anisotropic otherwise. A subspace $W$ of $V$ is called isotropic if there is an isotropic vector in $W$, otherwise $W$ is called anisotropic. Every isotropic quadratic space is universal, see [12, Ch. I, (4.5)]. The subspace $W$ is totally isotropic if $q(w)=0$ for all $w \in W$. If $W$ and $W^{\prime}$ are two maximal totally isotropic subspaces of $V$, then one has $\operatorname{dim} W=\operatorname{dim} W^{\prime}$ (see $[4,(12.11)]$ ). The dimension of a maximal totally isotropic subspace of $V$ is called the Witt index of $(V, q)$. By a quadratic plane we mean a 2-dimensional quadratic space $(\mathbb{E}, \varphi)$. Every quadratic plane has a basis $\{u, v\}$ such that $B(u, v)=1$. For such a basis we have $(u v)^{2}=u v+q(u) q(v) \cdot 1$, where this equality is considered in the Clifford algebra of $(\mathbb{E}, \varphi)$. Two quadratic planes $\left(\mathbb{E}_{1}, \varphi_{1}\right)$ and $\left(\mathbb{E}_{2}, \varphi_{2}\right)$ are isometric as bilinear spaces but not necessarily isometric as quadratic spaces. It is known that every quadratic space is an orthogonal sum of quadratic planes (see [12, Ch. I, (4.3)]). Up to isometry, there is a unique isotropic quadratic plane $(\mathbb{E}, \varphi)$, which is called a hyperbolic plane. For every hyperbolic plane $(\mathbb{E}, \varphi)$ we have $\varphi \simeq[1,0]$. We denote a hyperbolic plane by $\mathbb{H}$. A quadratic 
space $(V, q)$ is called hyperbolic if it is an orthogonal sum of hyperbolic planes. Every hyperbolic space has trivial Arf invariant.

An isometry $\tau$ of $(V, q)$ is called an involution in $O(V, q)$, if $\tau^{2}=$ id. Let $u \in V$ be an anisotropic vector and define a linear transformation $\tau_{u}: V \rightarrow V$ via $\tau_{u}(v)=v+\frac{B(v, u)}{q(u)} u$. It is easy to see that $\tau_{u}$ is an involution in $O(V, q)$. Note that if $B(u, v)=0$ then $\tau_{u}(v)=v$, also $\tau_{u}(u)=u$. We call $\tau_{u}$ the (orthogonal) reflection along $u$. In the literature, these isometries are also called orthogonal transvections. It is easy to see that if $u \in V$ is an anisotropic vector, then the restriction of the inner automorphism $\operatorname{Int}(u)$ of $C(V)$ to $V$ is just the reflection $\tau_{u}$. If $(V, q)$ is a quadratic space, then by a theorem due to Dieudonné, every isometry $\sigma$ of $(V, q)$ is a product of reflections except for the case that $\operatorname{dim} V=4,|F|=2$ and the Witt index of $(V, q)$ is 2 (see [4, (14.16)]). If $\sigma=\tau_{u_{1}} \cdots \tau_{u_{k}} \in O(V, q)$, then it can be shown that the class of $\Pi_{i=1}^{k} q\left(u_{i}\right)$ in the quotient group $F^{\times} / F^{\times 2}$, which is called the spinor norm of $\sigma$ and is denoted by $\theta(\sigma)$, is independent of the choice of the reflections $\tau_{u_{i}}$ 's, see [4, (14.17)]. In particular if $\sigma=\tau_{u}$ is a reflection, then the spinor norm of $\tau_{u}$ is the class of $q(u)$ in the quotient group $F^{\times} / F^{\times 2}$.

For an $F$-central simple algebra $A$ the integer $\sqrt{\operatorname{dim}_{F} A}$ is called the degree of $A$. An involution on $A$ is an anti-automorphism $\sigma$ of $A$ such that $\sigma^{2}=$ id. Let $(A, \sigma)$ be an $F$-central simple algebra with involution. Consider the subfield $K$ of $F$ consisting of the elements $a$ of $F$ such that $\sigma(a)=a$. It is known that either $F=K$, or $F / K$ is a separable field extension of degree 2 . In the first case we say $\sigma$ is of the first kind and in the second case we say $\sigma$ is of the second kind (or of unitary type).

Let $V$ be a finite dimensional vector space over a field $F$ and let $B$ be a nondegenerate bilinear form over $V$. The unique involution $\sigma_{B}$ on $\operatorname{End}_{F}(V)$, characterized by $B(x, f(y))=B\left(\sigma_{B}(f)(x), y\right)$ for every $x, y \in V$ and $f \in \operatorname{End}_{F}(V)$, is called the adjoint involution of $\operatorname{End}_{F}(V)$ with respect to $B$.

Let $(A, \sigma)$ be an $F$-central simple algebra of degree $n$ with involution of the first kind. If $L$ is a splitting field of $A$ and $V$ is an $n$-dimensional vector space over $L$, then there is a nondegenerate symmetric or antisymmetric bilinear form $B$ over $V$ such that $\left(A_{L}, \sigma_{L}\right) \simeq\left(\operatorname{End}_{L}(V), \sigma_{B}\right)$ (see [6, (2.1)]). An involution $\sigma$ of the first kind is said to be of symplectic type if for any splitting field $L$ of $A$ and any isomorphism $\left(A_{L}, \sigma_{L}\right) \simeq\left(\operatorname{End}_{L}(V), \sigma_{B}\right)$, the bilinear form $B$ is alternating. Otherwise we say that $\sigma$ is of orthogonal type. For short, we say that the central simple algebra with involution $(A, \sigma)$ is of orthogonal (resp. symplectic) type if $\sigma$ is of orthogonal (resp. symplectic) type.

A quaternion algebra over a field $F$ of characteristic 2 , is a 4-dimensional $F$-algebra $Q$ with a basis $\{1, i, j, k\}$ subject to the relations $i^{2}+i=\alpha \in F$, $j^{2}=\beta \in F^{\times}, i j=k$ and $i j+j i=j$. Note that these relations imply that $k^{2}=(i j)^{2}=\alpha \beta$. This algebra is denoted by $[\alpha, \beta)$ and we call $\{1, i, j, k\}$ a standard basis of $Q$. The map $\gamma: Q \rightarrow Q$ defined by $\gamma(a+b i+c j+d k)=$ $a+b(i+1)+c j+d k$ is an involution of the first kind on $Q$ which is called the canonical involution of $Q$. The canonical involution $\gamma$ is the unique involution on $Q$ such that $\gamma(x) x \in F$ for every $x \in Q$, see [14, Ch.8, (11.2)].

If $(A, \sigma)$ is an $F$-central simple algebra with involution, the set of alternating elements in $A$ is defined as follows:

$$
\operatorname{Alt}(A, \sigma)=\{a-\sigma(a) \mid a \in A\} .
$$


If $A$ is of even degree $n=2 m$ over $F$ and $\sigma$ is of orthogonal type, the determinant of $\sigma$ is the square class of the reduced norm of any alternating unit:

$$
\operatorname{det} \sigma=\operatorname{Nrd}_{A}(a) F^{\times 2} \in F^{\times} / F^{\times 2} \text { for } a \in \operatorname{Alt}(A, \sigma) \cap A^{*},
$$

and the discriminant of $\sigma$ is the signed determinant:

$$
\operatorname{disc} \sigma=(-1)^{m} \operatorname{det} \sigma \in F^{\times} / F^{\times 2} .
$$

Note that by $[7$, Lem. 2.1] or [6, (7.1)], the determinant and the discriminant are well-defined. Also if char $F=2$ the discriminant and the determinant coincide.

\section{Wiitala's characterization of involutions of or- thogonal groups in characteristic 2}

Definition 3.1. Let $(V, q, B)$ be a quadratic space over a field $F$ of characteristic 2 and let $\tau$ be an involution in $O(V, q)$. We call the subspace $\operatorname{Fix}(V, \tau)=\{v \in$ $V \mid \tau v=v\}$, the fixed subspace of $\tau$.

We state here the following definition from [17].

Definition 3.2. Let $(\mathbb{A}, q)$ be a quadratic space of dimension 4 over a field $F$ of characteristic 2. An involution $\tau \in O(\mathbb{A}, q)$ is called an interchange isometry if the fixed subspace $\operatorname{Fix}(\mathbb{A}, \tau)$ is a two dimensional totally isotropic space.

Remark 3.3. In [17], such maps are called "basic null involutions". In this work have preferred to use the term interchange isometry. Also in view of (3.6) below, this naming is not unreasonable.

Lemma 3.4. Let $(\mathbb{A}, q, B)$ be a quadratic space of dimension 4 over a field $F$ of characteristic 2 . If $\tau$ is an isometry of $(\mathbb{A}, q)$, then $\tau$ is an interchange isometry if and only if there is a basis $\{w, x, y, z\}$ of $\mathbb{A}$ such that $\{x, w\}$ is a basis of $\operatorname{Fix}(\mathbb{A}, \tau), q(w)=q(x)=0, \tau(y)=x+y, \tau(z)=w+z, B(x, z)=B(w, y)=1$ and all other pairs of vectors from this basis are orthogonal. In particular if $\tau$ is an interchange isometry of $(\mathbb{A}, q)$, then $(\mathbb{A}, q)$ is necessarily hyperbolic.

Proof. See [17, Lem. 2].

Remark 3.5. If $(\mathbb{A}, q, B)$ is a 4-dimensional quadratic space over a field $F$ of characteristic 2 and $\tau$ is an interchange isometry of $(\mathbb{A}, q)$, the basis $\{w, x, y, z\}$ of $\mathbb{A}$ with the properties stated in (3.4), can be chosen with the additional property that $q(y)=q(z)=0$; in fact set $\alpha=q(y), \beta=q(z), y^{\prime}=y+\alpha w$ and $z^{\prime}=z+\beta x$. It is easy to see that the basis $\left\{w, x, y^{\prime}, z^{\prime}\right\}$ has the desired property.

Proposition 3.6. Let $(\mathbb{A}, q, B)$ be a quadratic space of dimension 4 over a field $F$ of characteristic 2 and let $\tau$ be an isometry of $(\mathbb{A}, q)$. The following statements are equivalent: (1) $\tau$ is an interchange isometry. (2) There exist hyperbolic planes $\mathbb{H}_{1}$ and $\mathbb{H}_{2}$ in $\mathbb{A}$ such that $\mathbb{A}=\mathbb{H}_{1} \perp \mathbb{H}_{2}, \tau\left(\mathbb{H}_{1}\right)=\mathbb{H}_{2}$ and $\tau\left(\mathbb{H}_{2}\right)=\mathbb{H}_{1}$. (3) There exist quadratic planes $\mathbb{E}_{1}$ and $\mathbb{E}_{2}$ in $\mathbb{A}$ such that $\mathbb{A}=\mathbb{E}_{1} \perp \mathbb{E}_{2}$, $\tau\left(\mathbb{E}_{1}\right)=\mathbb{E}_{2}$ and $\tau\left(\mathbb{E}_{2}\right)=\mathbb{E}_{1}$. 
Proof. $(1) \Rightarrow(2)$ : Let $\{w, x, y, z\}$ be the basis of $\mathbb{A}$ with the properties stated in (3.5) and set $u_{1}=y, u_{2}=x+y=\tau\left(u_{1}\right), v_{1}=w+z$ and $v_{2}=z=\tau\left(v_{1}\right)$. Then

$$
\begin{aligned}
& B\left(u_{1}, u_{2}\right)=B(y, x+y)=0, \\
& B\left(u_{1}, v_{1}\right)=B(y, w+z)=B(y, w)+B(y, z)=1+0=1, \\
& B\left(u_{1}, v_{2}\right)=B(y, z)=0 \\
& B\left(v_{1}, u_{2}\right)=B\left(\tau\left(v_{2}\right), \tau\left(u_{1}\right)\right)=B\left(v_{2}, u_{1}\right)=0, \\
& B\left(v_{1}, v_{2}\right)=B(w+z, z)=0, \\
& B\left(u_{2}, v_{2}\right)=B\left(\tau\left(u_{1}\right), \tau\left(v_{1}\right)\right)=B\left(u_{1}, v_{1}\right)=1 .
\end{aligned}
$$

As $q\left(u_{1}\right)=q(y)=0$ and $q\left(v_{2}\right)=q(z)=0$, the subspaces $F u_{1}+F v_{1}$ and $F u_{2}+F v_{2}$ of $\mathbb{A}$ are hyperbolic. Let $\mathbb{H}_{1}=F u_{1}+F v_{1}$ and $\mathbb{H}_{2}=\tau\left(\mathbb{H}_{1}\right)=$ $F u_{2}+F v_{2}$. Then $\mathbb{H}_{1}=\mathbb{H}_{2}^{\perp}$. Finally as $\tau^{2}=\mathrm{id}$, we obtain $\tau\left(\mathbb{H}_{2}\right)=\mathbb{H}_{1}$.

$(2) \Rightarrow(3)$ is immediate.

$(3) \Rightarrow(1)$ : Set $u_{2}=\tau\left(u_{1}\right)$ and $v_{2}=\tau\left(v_{1}\right)$. Then $\mathbb{E}_{2}=F u_{2}+F v_{2}$. Let $v=a u_{1}+b u_{2}+c v_{1}+d v_{2} \in \operatorname{Fix}(\mathbb{A}, \tau)$, where $a, b, c, d \in F$. Then $a u_{2}+$ $b u_{1}+c v_{2}+d v_{1}=a u_{1}+b u_{2}+c v_{1}+d v_{2}$. Hence $a=b$ and $c=d$, i.e., $v=$ $a\left(u_{1}+u_{2}\right)+c\left(v_{1}+v_{2}\right)$. On the other hand the subspace $F\left(u_{1}+u_{2}\right)+F\left(v_{1}+v_{2}\right)$ of $\mathbb{A}$ is fixed by $\tau$. So $\operatorname{Fix}(\mathbb{A}, \tau)=F\left(u_{1}+u_{2}\right)+F\left(v_{1}+v_{2}\right)$. Finally we have $q\left(u_{1}+u_{2}\right)=q\left(v_{1}+v_{2}\right)=B\left(u_{1}+u_{2}, v_{1}+v_{2}\right)=0$, i.e., $\operatorname{Fix}(\mathbb{A}, \tau)$ is a 2-dimensional totally isotropic space. So $\tau$ is an interchange isometry.

Theorem 3.7. Let $(V, q, B)$ be a quadratic space over a field $F$ of characteristic 2 and let $\tau$ be an isometry of $(V, q)$. Then $\tau$ is an involution in $O(V, q)$ if and only if there is a decomposition of $V$ into regular subspaces $V=W \perp \mathbb{E}_{1} \perp$ $\cdots \perp \mathbb{E}_{r} \perp \mathbb{A}_{1} \perp \cdots \perp \mathbb{A}_{s}$ such that:

(a) $r$ and $s$ are nonnegative integers and for every $i, \operatorname{dim} \mathbb{E}_{i}=2$ and $\operatorname{dim} \mathbb{A}_{i}=$ 4 .

(b) $\tau$ maps each subspace $W, \mathbb{E}_{i}$ and $\mathbb{A}_{i}$ into itself.

(c) $\left.\tau\right|_{W}=\mathrm{id}$

(d) $\left.\tau\right|_{\mathbb{E}_{i}}$ is a reflection.

(e) $\left.\tau\right|_{\mathbb{A}_{i}}$ is an interchange isometry.

Proof. See [17, Thm. 1].

Definition 3.8. Let $(V, q, B)$ be a quadratic space over a field $F$ of characteristic 2 and let $\tau$ be an involution in $O(V, q)$. We call the decomposition $V=W \perp$ $\mathbb{E}_{1} \perp \cdots \perp \mathbb{E}_{r} \perp \mathbb{A}_{1} \perp \cdots \perp \mathbb{A}_{s}$ in (3.7), a Wiitala decomposition of $(V, \tau)$ and we call the subspace $W$, a maximal fixed orthogonal summand of $(V, \tau)$; in view of (3.9) below, this naming is justified. For every Wiitala decomposition $V=W \perp \mathbb{E}_{1} \perp \cdots \perp \mathbb{E}_{r} \perp \mathbb{A}_{1} \perp \cdots \perp \mathbb{A}_{s}$ of $(V, \tau)$, we have a decomposition $\tau=\mathrm{id}_{W} \perp \tau_{1} \perp \cdots \perp \tau_{r} \perp \tau_{1}^{\prime} \perp \cdots \perp \tau_{s}^{\prime}$ which is called a Wiitala decomposition of $\tau$ where $\tau_{i}$ is a reflection of $\mathbb{E}_{i}$ for $i=1, \cdots, r$ and $\tau_{i}^{\prime}$ is an interchange isometry of $\mathbb{A}_{i}$ for $i=1, \cdots, s$ (i.e., the integers $r$ and $s$ are the respective number of reflections and interchange isometries in the above Wiitala decomposition of $\tau$ ). 
Proposition 3.9. Let $(V, q, B)$ be a quadratic space over a field $F$ of characteristic 2 and let $\tau$ be an involution in $O(V, q)$. Let $U \subseteq V$ be an orthogonal summand of $V$ such that $\left.\tau\right|_{U}=\mathrm{id}$. Then there exists a maximal fixed orthogonal summand $W$ of $(V, \tau)$ such that $U \subseteq W$. In particular $\operatorname{dim} W$ is uniquely determined by $\tau$.

Proof. Let $U^{\prime}$ be an orthogonal complement of $U$, i.e., $V=U \perp U^{\prime}$. Then $\left.\tau\right|_{U^{\prime}}$ is an involution in $O\left(U^{\prime},\left.q\right|_{U^{\prime}}\right)$. Let $U^{\prime}=W_{1} \perp \mathbb{E}_{1} \perp \cdots \perp \mathbb{E}_{r} \perp \mathbb{A}_{1} \perp \cdots \perp \mathbb{A}_{s}$ be a Wiitala decomposition of $\left(U^{\prime},\left.\tau\right|_{U^{\prime}}\right)$ and set $W=U \perp W_{1}$. Then $V=W \perp$ $\mathbb{E}_{1} \perp \cdots \perp \mathbb{E}_{r} \perp \mathbb{A}_{1} \perp \cdots \perp \mathbb{A}_{s}$ is a Wiitala decomposition of $(V, \tau)$. So $W$ is a maximal fixed orthogonal summand of $(V, \tau)$ and $U \subseteq W$.

Remark 3.10. Let $(V, q, B)$ be a quadratic space over a field $F$ of characteristic 2 and let $\tau$ be an involution in $O(V, q)$. Let $W$ be a maximal fixed orthogonal summand of $(V, \tau)$. Then $W$ is not necessarily unique up to isometry. For example let $\left(\mathbb{E}_{i}, \varphi_{i}\right) \simeq[\alpha, \beta], i=1,2$, be an anisotropic quadratic plane over $F$ with standard symplectic basis $\left\{u_{i}, v_{i}\right\}$. Set $(V, q)=\left(\mathbb{E}_{1}, \varphi_{1}\right) \perp\left(\mathbb{E}_{2}, \varphi_{2}\right)$ and $\tau=\operatorname{id}_{\mathbb{E}_{1}} \perp \tau_{u_{2}}$ where $\tau_{u_{2}}$ is the reflection of $\mathbb{E}_{2}$ along $u_{2}$. Then $\tau$ is an involution in $O(V, q)$ and $W_{1}=\mathbb{E}_{1}$ is a maximal fixed orthogonal summand of $(V, \tau)$. Now set $W=F\left(u_{1}+u_{2}\right)+F v_{1}$ and $\mathbb{E}=F u_{2}+F\left(v_{1}+v_{2}\right)$. Then $V=W \perp \mathbb{E}$ and $\left.\tau\right|_{W}=$ id. Also $\left.\tau\right|_{\mathbb{E}}$ is the reflection along $u_{2}$, because $\tau\left(u_{2}\right)=u_{2}=\tau_{u_{2}}\left(u_{2}\right)$ and $\tau\left(v_{1}+v_{2}\right)=v_{1}+\left(v_{2}+\frac{1}{q\left(u_{2}\right)} u_{2}\right)=\left(v_{1}+v_{2}\right)+\frac{B\left(v_{1}+v_{2}, u_{2}\right)}{q\left(u_{2}\right)} u_{2}=\tau_{u_{2}}\left(v_{2}\right)$. So $V=W \perp \mathbb{E}$ is a Wiitala decomposition of $(V, \tau)$ and $W$ is a maximal fixed orthogonal summand of $(V, \tau)$. Now we have $q\left(u_{1}+u_{2}\right)=\alpha+\alpha=0$, so $W$ is isotropic and $W_{1} \neq W$.

Definition 3.11. Let $(V, q, B)$ be a quadratic space over a field $F$ of characteristic 2 and let $\tau$ be an involution in $O(V, q)$. Let $V=W \perp \mathbb{E}_{1} \perp \cdots \perp \mathbb{E}_{r} \perp$ $\mathbb{A}_{1} \perp \cdots \perp \mathbb{A}_{s}$ be a Wiitala decomposition of $(V, \tau)$. We call a symplectic basis

$$
\left\{u_{1}, v_{1}, \cdots, u_{t}, v_{t}, u_{1}^{\prime}, v_{1}^{\prime}, \cdots, u_{r}^{\prime}, v_{r}^{\prime}, u_{1}^{\prime \prime}, v_{1}^{\prime \prime}, \cdots, u_{2 s}^{\prime \prime}, v_{2 s}^{\prime \prime}\right\}
$$

of $V$ a $\tau$-symplectic basis of $(V, \tau)$ if it satisfies the following conditions:

(a) The set $\left\{u_{1}, v_{1}, \cdots, u_{t}, v_{t}\right\}$ is a symplectic basis of the subspace $W$ (so $\left.t=\frac{1}{2} \operatorname{dim} W\right)$.

(b) For $1 \leqslant i \leqslant r,\left\{u_{i}^{\prime}, v_{i}^{\prime}\right\}$ is a symplectic basis of the subspace $\mathbb{E}_{i}$ such that $q\left(u_{i}^{\prime}\right) \neq 0, \tau\left(u_{i}^{\prime}\right)=u_{i}^{\prime}$ and $\tau\left(v_{i}^{\prime}\right)=v_{i}^{\prime}+\frac{1}{q\left(u_{i}^{\prime}\right)} u_{i}^{\prime}$ (i.e., $\left.\left.\tau\right|_{\mathbb{E}_{i}}=\tau_{u_{i}^{\prime}}\right)$.

(c) For $1 \leqslant i \leqslant s,\left\{u_{2 i-1}^{\prime \prime}, v_{2 i-1}^{\prime \prime}, u_{2 i}^{\prime \prime}, v_{2 i}^{\prime \prime}\right\}$ is a basis of the subspace $\mathbb{A}_{i}$ such that the subspace $F u_{2 i-1}^{\prime \prime}+F v_{2 i-1}^{\prime \prime}$ is perpendicular to the subspace $F u_{2 i}^{\prime \prime}+F v_{2 i}^{\prime \prime}$, $\tau\left(u_{2 i-1}^{\prime \prime}\right)=u_{2 i}^{\prime \prime}, \tau\left(u_{2 i}^{\prime \prime}\right)=u_{2 i-1}^{\prime \prime}, \tau\left(v_{2 i-1}^{\prime \prime}\right)=v_{2 i}^{\prime \prime}$ and $\tau\left(v_{2 i}^{\prime \prime}\right)=v_{2 i-1}^{\prime \prime}$ (i.e., $\left.\tau\right|_{\mathbb{A}_{i}}$ is an interchange isometry).

For simplicity, we denote the above $\tau$-symplectic basis of $V$ by $\left\{u_{1}, v_{1}, \cdots, u_{n}, v_{n}\right\}$ where $n=t+r+2 s$ and for $t+1 \leqslant i \leqslant t+r$, we have $u_{i}=u_{i-t}^{\prime}$ and $v_{i}=v_{i-t}^{\prime}$ and for $t+r+1 \leqslant i \leqslant t+r+2 s=n$, we have $u_{i}=u_{i-t-r}^{\prime \prime}$ and $v_{i}=v_{i-t-r}^{\prime \prime}$.

Remark 3.12. Let $(\mathbb{E}, \varphi)$ be a quadratic plane over a field $F$ of characteristic 2 and let $\tau \neq$ id be an involution in $O(\mathbb{E}, \varphi)$. Then $\tau$ is a reflection (see [17, Lem. 1]). In particular for every quadratic plane $(\mathbb{E}, \varphi)$ and every involution $\tau \in O(\mathbb{E}, \varphi)$, there exists an anisotropic vector $u \in \mathbb{E}$ such that $\tau(u)=u$. 
Lemma 3.13. Let $(\mathbb{E}, \varphi)$ be a quadratic plane over a field $F$ of characteristic 2 and let $\tau$ be an involution in $O(\mathbb{E}, \varphi)$. Let $t=\operatorname{dim} \operatorname{Fix}(\mathbb{E}, \tau)-\frac{1}{2} \operatorname{dim} \mathbb{E}$ and let $u \in \mathbb{E}$ be an anisotropic vector such that $\tau(u)=u$. Then for every $x \in \mathbb{E}$, we have $\tau(x)=x+(t+1) B(x, u) u^{-1}$.

Proof. If $\tau=\mathrm{id}$, then $t=1$ and $\tau(x)=x=x+(t+1) B(x, u) u^{-1}$. Otherwise by (3.12), we have $\tau=\tau_{u}, t=0$ and $\tau(x)=x+B(x, u) u^{-1}=x+(t+$ 1) $B(x, u) u^{-1}$.

Lemma 3.14. Let $(V, q, B)$ be a quadratic space over a field $F$ of characteristic 2 and let $\tau$ be an involution in $O(V, q)$.

(a) If $\operatorname{dim} V=2$ then we have $\tau=$ id if and only if for every $v \in V$ we have $B(v, \tau(v))=0$.

(b) If $\operatorname{dim} V=4$ and $\tau \neq \mathrm{id}$, then $\tau$ is an interchange isometry if and only if for every $v \in V$, we have $B(v, \tau(v))=0$.

(c) We have $B(v, \tau(v))=0$ for every $v \in V$ if and only if there is no reflection in any Wiitala decomposition of $\tau$.

Proof. (a) If $\tau=\mathrm{id}$, the conclusion is evident. Conversely suppose that $\tau \neq \mathrm{id}$. By (3.12) there exists an anisotropic vector $u \in V$ such that $\tau$ is the reflection along $u$, i.e., $\tau=\tau_{u}$. Let $v \in V$ with $B(u, v)=1$. Then $B(v, \tau(v))=B(v, v+$ $\left.\frac{1}{q(u)} u\right)=\frac{1}{q(u)} \neq 0$.

(b) Let $\tau$ be an interchange isometry. Choose a basis $\{w, x, y, z\}$ of $V$ with the properties stated in (3.4). Let $v \in V$ and write $v=a w+b x+c y+d z$ where $a, b, c, d \in F$. We have

$$
\begin{aligned}
B(v, \tau(v)) & =B(a w+b x+c y+d z, a w+b x+c(y+x)+d(z+w)) \\
& =B(a w+b x+c y+d z,(a+d) w+(b+c) x+c y+d z) \\
& =a c+b d+c(a+d)+d(b+c)=0
\end{aligned}
$$

and we are done. Conversely suppose that $B(v, \tau(v))=0$ for every $v \in V$. As $\tau \neq \mathrm{id}$, if $\tau$ is not an interchange isometry then by (3.7) there is necessarily a quadratic plane $\mathbb{E} \subseteq V$ such that $\left.\tau\right|_{\mathbb{E}}$ is a reflection. But $(a)$ implies that there exists $v \in \mathbb{E}$ such that $B(v, \tau(v)) \neq 0$, this contradicts the hypothesis.

(c) If there is a reflection in a Wiitala decomposition of $\tau$, then there exists $v \in V$ such that $B(v, \tau(v)) \neq 0$. Conversely suppose that there is no reflection in any Wiitala decomposition of $\tau$. By (3.7), we can write $V=W \perp \mathbb{A}_{1} \perp \cdots \perp$ $\mathbb{A}_{s}$, where $\left.\tau\right|_{W}=$ id and $\left.\tau\right|_{\mathbb{A}_{i}}$ is an interchange isometry. For every $v \in V$ we can write $v=v_{0}+v_{1}+\cdots+v_{s}$ where $v_{0} \in W$ and $v_{i} \in \mathbb{A}_{i}, i=1, \ldots, s$. Then using $(b)$ we obtain

$$
\begin{aligned}
B(v, \tau(v)) & =B\left(v_{0}, \tau\left(v_{0}\right)\right)+B\left(v_{1}, \tau\left(v_{1}\right)\right)+\cdots+B\left(v_{s}, \tau\left(v_{s}\right)\right) \\
& =0+0+\cdots+0=0 .
\end{aligned}
$$

Proposition 3.15. Let $(V, q, B)$ be a quadratic space of dimension 6 over a field $F$ of characteristic 2 and let $\tau$ be an involution in $O(V, q)$. Suppose that $V$ has a decomposition of the form $V=\mathbb{E} \perp \mathbb{A}$ where $\mathbb{E}$ is a quadratic plane 
in $V,\left.\tau\right|_{\mathbb{E}}$ is a reflection and $\left.\tau\right|_{\mathbb{A}}$ is an interchange isometry. Then there is a decomposition of $V$ of the form $V=\mathbb{E}_{1} \perp \mathbb{E}_{2} \perp \mathbb{E}_{3}$ such that $\mathbb{E}_{1}, \mathbb{E}_{2}$ and $\mathbb{E}_{3}$ are quadratic planes in $V$ and for every $i=1,2,3,\left.\tau\right|_{\mathbb{E}_{i}}$ is a reflection.

Proof. See [17, Thm. 2].

Theorem 3.16. Let $(V, q, B)$ be a quadratic space over a field $F$ of characteristic 2 and let $\tau$ be an involution in $O(V, q)$. Then there is a decomposition of $(V, \tau)$ in exactly one of the following forms:

(a) $V=W \perp \mathbb{E}_{1} \perp \cdots \perp \mathbb{E}_{r}$ where $\left.\tau\right|_{W}=\mathrm{id}, \mathbb{E}_{i}$ is a quadratic subplane of $V$ and $\left.\tau\right|_{\mathbb{E}_{i}}$ is a reflection for every $1 \leqslant i \leqslant r$.

(b) $V=W \perp \mathbb{A}_{1} \perp \cdots \perp \mathbb{A}_{s}$ where $\left.\tau\right|_{W}=\mathrm{id}, \operatorname{dim} \mathbb{A}_{i}=4$ and $\left.\tau\right|_{\mathbb{A}_{i}}$ is an interchange isometry for every $1 \leqslant i \leqslant s$.

Furthermore the type of decomposition occurring and the numbers $r$ and $s$ are uniquely determined by $\tau$.

Proof. See [17, Thm. 2].

Definition 3.17. Let $(V, q, B)$ be a quadratic space over a field $F$ of characteristic 2 and let $\tau$ be an involution in $O(V, q)$. We say that $\tau$ is of reflectional (resp. interchanging) kind if $V$ has a decomposition of the form (a) (resp. (b)) in (3.16).

The following result is a restatement of $(3.14(\mathrm{c}))$ :

Corollary 3.18. Let $(V, q, B)$ be a quadratic space over a field $F$ of characteristic 2 and let $\tau$ be an involution in $O(V, q)$. Then $\tau$ is of interchanging kind if and only if $B(v, \tau(v))=0$ for every $v \in V$.

Proposition 3.19. Let $(V, q)$ be a quadratic space over a field $F$ of characteristic 2 and let $\tau$ be an involution in $O(V, q)$. Let $r$ be the number of reflections in any Wiitala decomposition of $(V, \tau)$. Then $r \equiv \operatorname{dim} \operatorname{Fix}(V, \tau) \bmod 2$ and $\operatorname{dim} \operatorname{Fix}(V, \tau)=\frac{1}{2} \operatorname{dim} W+\frac{1}{2} \operatorname{dim} V$ where $W$ is a maximal fixed orthogonal summand of $(V, \tau)$. In particular $\operatorname{dim} \operatorname{Fix}(V, \tau) \geqslant \frac{1}{2} \operatorname{dim} V$. Also we have $\operatorname{dim} \operatorname{Fix}(V, \tau)=\frac{1}{2} \operatorname{dim} V$ if and only if $W=0$.

Proof. Let $V=W \perp \mathbb{E}_{1} \perp \cdots \perp \mathbb{E}_{r} \perp \mathbb{A}_{1} \perp \cdots \perp \mathbb{A}_{s}$ be a Wiitala decomposition of $(V, \tau)$. Then for $1 \leqslant i \leqslant r,\left.\tau\right|_{\mathbb{E}_{i}}$ is a reflection, so the fixed subspace of $\left.\tau\right|_{\mathbb{E}_{i}}$ is one dimensional. Similarly for $1 \leqslant i \leqslant s,\left.\tau\right|_{\mathbb{A}_{i}}$ is an interchange isometry, so the fixed subspace of $\left.\tau\right|_{\mathbb{A}_{i}}$ is two dimensional. So the fixed subspace of the restriction of $\tau$ to $\mathbb{E}_{1} \perp \cdots \perp \mathbb{E}_{r} \perp \mathbb{A}_{1} \perp \cdots \perp \mathbb{A}_{s}$ has dimension $r+2 s$ and we obtain $\operatorname{dim} \operatorname{Fix}(V, \tau)=r+2 s+\operatorname{dim} W=\left(r+2 s+\frac{1}{2} \operatorname{dim} W\right)+\frac{1}{2} \operatorname{dim} W=$ $\frac{1}{2} \operatorname{dim} V+\frac{1}{2} \operatorname{dim} W$. Finally since $W$ is a regular subspace of $v, \operatorname{dim} W$ is even, so $r \equiv \operatorname{dim} \operatorname{Fix}(V, \tau) \bmod 2$.

Remark 3.20. Let $(V, q, B)$ be a quadratic space over a field $F$ of characteristic 2 and let $\tau$ be an involution in $O(V, q)$. If $\operatorname{dim} \operatorname{Fix}(V, \tau)=\frac{1}{2} \operatorname{dim} V$ and $\tau$ is of interchanging kind, then by $(3.19),(V, \tau)$ has trivial maximal fixed orthogonal summand. So by (3.16), $V=\mathbb{A}_{1} \perp \cdots \perp \mathbb{A}_{s}$. By (3.6), $V$ is a hyperbolic space. In particular the Arf invariant of $q$ is trivial. If $\tau$ is of reflectional kind, depending on $(V, q), \Delta(q)$ can be trivial or nontrivial. For example if $V=\mathbb{H}$ and $\tau$ is a reflection of $V$, then $\Delta(q)$ is trivial, while if $V=\mathbb{E}$ is the quadratic plane $[1, \delta]$ with $\delta \in F \backslash \wp(F)$ and $\tau$ is a reflection of $V$, then $\Delta(q)=\delta+\wp(F) \in F / \wp(F)$ is nontrivial. 


\section{Generalities on some natural involutions of a Clifford algebra}

Remark 4.1. Let $(V, q)$ be a quadratic space over a field $F$ of characteristic 2 and let $C(V)$ be the Clifford algebra of $(V, q)$. If $\tau: V \rightarrow V$ is an involution in $O(V, q)$, then $\tau$ induces an involution $J_{\tau}^{q}$ on $C(V)$ such that $J_{\tau}^{q}(v)=\tau(v)$ for every $v \in V$. Also if $\sigma$ is an involution on $C(V)$ such that $\sigma(V)=V$, then $\tau=\left.\sigma\right|_{V}$ is involution in $O(V, q)$. We call $J_{\tau}^{q}$ the involution induced by $\tau$ on $C(V)$ and we usually abbreviate $J_{\tau}^{q}$ by $J_{\tau}$.

Remark 4.2. Let $(\mathbb{E}, \varphi)$ be a quadratic plane over a field $F$ of characteristic 2 and let $\tau=\tau_{u}$ be a reflection along some anisotropic vector $u \in \mathbb{E}$. Extend $\{u\}$ to a symplectic basis $\{u, v\}$ of $\mathbb{E}$. Consider the involution $J_{\tau}$ on $C(\mathbb{E})$. We have $J_{\tau}(u)=u, J_{\tau}(v)=v+\frac{1}{q(u)} u$ and $J_{\tau}(u v)=\tau(v) \tau(u)=\left(v+\frac{1}{q(u)} u\right) u=$ $v u+1=u v$. So if $x=a+b u+c v+d u v \in C(\mathbb{E})$ where $a, b, c, d \in F$, then we get $x-J_{\tau}(x)=\frac{c}{q(u)} u$, i.e., $\operatorname{Alt}\left(C(\mathbb{E}), J_{\tau}\right)=F u$. In particular we have $x-J_{\tau}(x) \in F$ if and only if $x=J_{\tau}(x)$.

Proposition 4.3. Let $(\mathbb{E}, \varphi, B)$ be a quadratic plane over a field $F$ of characteristic 2 and let $\tau$ be an involution in $O(\mathbb{E}, \varphi)$. The involution $J_{\tau}$ on $C(\mathbb{E})$ is of symplectic type if and only if $\tau=$ id if and only if $\mathrm{D}(\tau)=0 \in \mathbb{Z} / 2 \mathbb{Z}$. In other words $J_{\tau}$ is of orthogonal type if and only if $\tau$ is a reflection.

Proof. Suppose that $\tau=\mathrm{id}$ and $\{x, y\}$ is a basis of $\mathbb{E}$ such that $B(x, y)=1$. Then $J_{\tau}(x y)=\tau(y) \tau(x)=y x=x y+1$, so $1 \in \operatorname{Alt}\left(C(\mathbb{E}), J_{\tau}\right)$ and by $[6,(2.6)]$, $J_{\tau}$ is of symplectic type.

Conversely suppose that $\tau \neq \mathrm{id}$. Then by (3.12), $\tau$ is a reflection along some anisotropic vector $u \in \mathbb{E}$, i.e., $\tau=\tau_{u}$. By $(4.2), \operatorname{Alt}\left(C(\mathbb{E}), J_{\tau}\right)=F u$, hence $1 \notin \operatorname{Alt}\left(C(\mathbb{E}), J_{\tau}\right)$. So by $[6,(2.6)], J_{\tau}$ is of orthogonal type.

This proves the first part of the proposition. For the second part, if $\tau \neq$ id is an involution in $O(\mathbb{E}, \varphi)$, then $\tau$ is a reflection and for any reflection $\tau$ we have $\mathrm{D}(\tau)=1 \in \mathbb{Z} / 2 \mathbb{Z}($ see $[14$, Ch. $9,(4.11)])$.

Lemma 4.4. Let $\left(V_{1}, q_{1}\right)$ and $\left(V_{2}, q_{2}\right)$ be quadratic spaces over a field $F$ of characteristic 2. If $\tau_{1}$ and $\tau_{2}$ are involutions in $O\left(V_{1}, q_{1}\right)$ and $O\left(V_{2}, q_{2}\right)$, respectively, then $\left(C\left(V_{1}\right), J_{\tau_{1}}\right) \otimes\left(C\left(V_{2}\right), J_{\tau_{2}}\right) \simeq\left(C\left(V_{1} \perp V_{2}\right), J_{\tau_{1} \perp \tau_{2}}\right)$.

Proof. It is easy to see that the linear map $\varphi: V_{1} \perp V_{2} \rightarrow C\left(V_{1}\right) \otimes C\left(V_{2}\right)$ defined by $\varphi\left(v_{1}+v_{2}\right)=v_{1} \otimes 1+1 \otimes v_{2}$ where $v_{1} \in V_{1}$ and $v_{2} \in V_{2}$ can be extended to an isomorphism $\varphi: C\left(V_{1} \perp V_{2}\right) \simeq C\left(V_{1}\right) \otimes C\left(V_{2}\right)$ (cf. [4, p. 120]). So it remains to show that $\varphi \circ J_{\tau_{1} \perp \tau_{2}}=\left(J_{\tau_{1}} \otimes J_{\tau_{2}}\right) \circ \varphi$. It is enough to check that $\varphi \circ J_{\tau_{1} \perp \tau_{2}}(w)=\left(J_{\tau_{1}} \otimes J_{\tau_{2}}\right) \circ \varphi(w)$ for every $w \in V_{1} \perp V_{2}$. Write $w=v_{1}+v_{2}$ where $v_{1} \in V_{1}$ and $v_{2} \in V_{2}$, then

$$
\begin{aligned}
\varphi \circ J_{\tau_{1} \perp \tau_{2}}(w) & =\varphi \circ J_{\tau_{1} \perp \tau_{2}}\left(v_{1}+v_{2}\right)=\varphi\left(\tau_{1}\left(v_{1}\right)+\tau_{2}\left(v_{2}\right)\right) \\
& =\tau_{1}\left(v_{1}\right) \otimes 1+1 \otimes \tau_{2}\left(v_{2}\right)=\left(J_{\tau_{1}} \otimes J_{\tau_{2}}\right)\left(v_{1} \otimes 1+1 \otimes v_{2}\right) \\
& =\left(J_{\tau_{1}} \otimes J_{\tau_{2}}\right) \circ \varphi\left(v_{1}+v_{2}\right)=\left(J_{\tau_{1}} \otimes J_{\tau_{2}}\right) \circ \varphi(w) .
\end{aligned}
$$

Lemma 4.5. Let $(V, q)$ be a quadratic space over a field $F$ of characteristic 2 and let $\tau$ be an involution in $O(V, q)$. Then for every $a \in F^{\times}$we have $\left(C_{0}(V, q), J_{\tau}^{q}\right) \cong\left(C_{0}(V, a \cdot q), J_{\tau}^{a \cdot q}\right)$. 
Proof. Define a bilinear map $\psi: V \times V \rightarrow C_{0}(V, a \cdot q)$ via $\psi(x, y)=a^{-1} \cdot x y$ for every $x, y \in V$. For every $x, y, z \in V$ we have $\psi(x, y) \psi(y, z)=\left(a^{-1}\right.$. $x y)\left(a^{-1} \cdot y z\right)=a^{-2} a q(y) \cdot x z=a^{-1} q(y) \cdot x z=q(y) \cdot \psi(x, z)$ and $\psi(x, x)=$ $a^{-1} \cdot x^{2}=a^{-1} a \cdot q(x)=q(x) \cdot 1$. So $\psi$ is an even Clifford map and by (A.5), there exists an $F$-algebra homomorphism $\Psi: C_{0}(V, q) \rightarrow C_{0}(V, a \cdot q)$ induced by $\Psi(x y)=a^{-1} \cdot x y$ for every $x, y \in V$. The map $\psi$ is clearly an isomorphism.

Let $\left\{u_{1}, v_{1}, \cdots, u_{n}, v_{n}\right\}$ be a symplectic basis of $(V, q)$. For every $1 \leqslant i, j \leqslant n$ we have

$\Psi \circ J_{\tau}^{q}\left(u_{i} u_{j}\right)=\Psi\left(\tau\left(u_{j}\right) \tau\left(u_{i}\right)\right)=a^{-1} \tau\left(u_{j}\right) \tau\left(u_{i}\right)=J_{\tau}^{a \cdot q}\left(a^{-1} u_{i} u_{j}\right)=J_{\tau}^{a \cdot q} \circ \Psi\left(u_{i} u_{j}\right)$.

Similarly we have the following relations

$$
\begin{aligned}
& \Psi \circ J_{\tau}^{q}\left(v_{i} v_{j}\right)=J_{\tau}^{a \cdot q} \circ \Psi\left(v_{i} v_{j}\right), \\
& \Psi \circ J_{\tau}^{q}\left(u_{i} v_{j}\right)=J_{\tau}^{a \cdot q} \circ \Psi\left(u_{i} v_{j}\right) .
\end{aligned}
$$

So $\Psi \circ J_{\tau}^{q}(x)=J_{\tau}^{a \cdot q} \circ \Psi(x)$ for every $x \in C_{0}(V, q)$. This completes the proof.

Proposition 4.6. Let $(\mathbb{A}, q)$ be a quadratic space of dimension 4 over a field $F$ of characteristic 2 and let $\tau$ be an interchange isometry of $(\mathbb{A}, q)$. Then the involution $J_{\tau}$ on $C(\mathbb{A})$ is of orthogonal type.

Proof. Let $(\mathbb{E}, \varphi)$ be a quadratic plane over $F$ and let $\rho$ be a reflection of $(\mathbb{E}, \varphi)$. Set $V=\mathbb{E} \perp \mathbb{A}$. Then $\tau \perp \rho$ is an involution in $O(V, \varphi \perp q)$. By (3.15), there is a decomposition $V=\mathbb{E}_{1} \perp \mathbb{E}_{2} \perp \mathbb{E}_{3}$ such that $\tau_{i}:=\left.(\tau \perp \rho)\right|_{\mathbb{E}_{i}}$ is a reflection for every $i=1,2,3$. Hence by (4.3), $J_{\tau_{i}}$ is an involution of orthogonal type on $C\left(\mathbb{E}_{i}\right)$. Using (4.4) we obtain $\left(C(V), J_{\tau \perp \rho}\right) \simeq\left(C\left(\mathbb{E}_{1}\right), J_{\tau_{1}}\right) \otimes\left(C\left(\mathbb{E}_{2}\right), J_{\tau_{2}}\right) \otimes\left(C\left(\mathbb{E}_{3}\right), J_{\tau_{3}}\right)$, and by $[6,(2.23)], J_{\tau \perp \rho}$ is of orthogonal type.

On the other hand we have $\left(C(V), J_{\tau \perp \rho}\right) \simeq\left(C(\mathbb{A}), J_{\tau}\right) \otimes\left(C(\mathbb{E}), J_{\rho}\right)$. Since $\left(C(V), J_{\tau \perp \rho}\right)$ is of orthogonal type, again by $[6,(2.23)], J_{\tau}$ is of orthogonal type on $C(\mathbb{A})$ and we are done.

Theorem 4.7. Let $(V, q)$ be a quadratic space over a field $F$ of characteristic 2 and let $\tau$ be an involution in $O(V, q)$. Then the involution $J_{\tau}$ on $C(V)$ is of orthogonal type if and only if $(V, \tau)$ has trivial maximal fixed orthogonal summand if and only if $\operatorname{dim} \operatorname{Fix}(V, \tau)=\frac{1}{2} \operatorname{dim} V$.

Proof. Let $V=W \perp \mathbb{E}_{1} \perp \cdots \perp \mathbb{E}_{r} \perp \mathbb{A}_{1} \perp \cdots \perp \mathbb{A}_{s}$ be a Wiitala decomposition of $(V, \tau)$. We have $\left(C(V), J_{\tau}\right) \simeq\left(C(W), J_{\text {id }}\right) \otimes\left(C\left(W^{\prime}\right), J_{\left.\tau\right|_{W^{\prime}}}\right)$ where $W^{\prime}=\mathbb{E}_{1} \perp \cdots \perp \mathbb{E}_{r} \perp \mathbb{A}_{1} \perp \cdots \perp \mathbb{A}_{s}$. The conclusion follows from (4.3), (4.4), (4.6) and $[6,(2.23)]$. The equivalence of the last two statements follows from (3.19).

Corollary 4.8. Let $(V, q)$ be a quadratic space over a field $F$ of characteristic 2 .

(a) The involution $J_{\mathrm{id}}$ on $C(V)$ is of symplectic type.

(b) Let $\tau$ be a reflection of $V$. If $\operatorname{dim} V=2$, then the involution $J_{\tau}$ on $C(V)$ is of orthogonal type. If $\operatorname{dim} V \geqslant 4$, then $J_{\tau}$ is of symplectic type.

Proof. (a) We have $\operatorname{dim} \operatorname{Fix}(V$, id $)=\operatorname{dim} V>\frac{1}{2} \operatorname{dim} V$. So by (4.7), $J_{\text {id }}$ is of symplectic type.

(b) If $\operatorname{dim} V=2$, then by (4.3), $J_{\tau}$ is of orthogonal type. If $\operatorname{dim} V \geqslant 4$, then $\operatorname{dim} \operatorname{Fix}(V, \tau)=\operatorname{dim} V-1>\frac{1}{2} \operatorname{dim} V$ and by (4.7), $J_{\tau}$ is of symplectic type. 
Proposition 4.9. Let $(V, q)$ be a quadratic space over a field $F$ of characteristic 2 and let $\tau$ be an involution in $O(V, q)$. Then $\mathrm{D}(\tau)$ is equal to the class of $\operatorname{dim} \operatorname{Fix}(V, \tau)$ in $\mathbb{Z} / 2 \mathbb{Z}$.

Proof. Let $V=W \perp \mathbb{E}_{1} \perp \cdots \perp \mathbb{E}_{r} \perp \mathbb{A}_{1} \perp \cdots \perp \mathbb{A}_{s}$ be a Wiitala decomposition of $(V, \tau)$ and let $\left\{u_{1}, v_{1}, \cdots, u_{t}, v_{t}, u_{1}^{\prime}, v_{1}^{\prime}, \cdots, u_{r}^{\prime}, v_{r}^{\prime}, u_{1}^{\prime \prime}, v_{1}^{\prime \prime}, \cdots, u_{2 s}^{\prime \prime}, v_{2 s}^{\prime \prime}\right\}$ be a $\tau$-symplectic basis with respect to this decomposition and set $z=u_{1} v_{1}+$ $\cdots+u_{t} v_{t}+u_{1}^{\prime} v_{1}^{\prime}+\cdots+u_{r}^{\prime} v_{r}^{\prime}+u_{1}^{\prime \prime} v_{1}^{\prime \prime}+\cdots+u_{2 s}^{\prime \prime} v_{2 s}^{\prime \prime}$. Let $I_{\tau}$ be the automorphism of $C_{0}(V)$ induced by $\tau$. Then $\left.I_{\tau}\right|_{W}=$ id. Also for $1 \leqslant i \leqslant r$, we have $I_{\tau}\left(u_{i}^{\prime} v_{i}^{\prime}\right)=u_{i}^{\prime}\left(v_{i}^{\prime}+\frac{1}{q\left(u_{i}^{\prime}\right)} u_{i}^{\prime}\right)=u_{i}^{\prime} v_{i}^{\prime}+1$. Finally for $1 \leqslant i \leqslant s$, we have $I_{\tau}\left(u_{2 i-1}^{\prime \prime} v_{2 i-1}^{\prime \prime}+u_{2 i}^{\prime \prime} v_{2 i}^{\prime \prime}\right)=u_{2 i}^{\prime \prime} v_{2 i}^{\prime \prime}+u_{2 i-1}^{\prime \prime} v_{2 i-1}^{\prime \prime}$. Hence $I_{\tau}(z)=z+r \cdot 1$, where $r$ is the number of reflections in the above Wiitala decomposition of $\tau$. Note that by (3.19) the number of reflections modulo two is independent of the decomposition of $\tau$. Since $Z\left(C_{0}(V)\right)$ is generated by 1 and $z$ over $F$ (see [4, (13.12)]), the Dickson invariant $\mathrm{D}(\tau)$ is equal to the class of $r$ in $\mathbb{Z} / 2 \mathbb{Z}$. On the other hand by $(3.19), r \equiv \operatorname{dim} \operatorname{Fix}(V, \tau) \bmod 2$ and therefore, $\mathrm{D}(\tau)$ is equal to the class of $\operatorname{dim} \operatorname{Fix}(V, \tau)$ in $\mathbb{Z} / 2 \mathbb{Z}$.

Lemma 4.10. Let $F$ be a field of characteristic 2 and let $A$ a be central simple algebra over $F$. If $[A: F]=n^{2}$ is even and $x \in A$ is an element such that $x^{2}=\alpha \in F$, then $\operatorname{Nrd}(x)=\alpha^{\frac{n}{2}}$.

Proof. We have $\operatorname{Nrd}(x)^{2}=\operatorname{Nrd}(\alpha)=\alpha^{n}$. Since $n$ is even and char $F=2$, we get $\operatorname{Nrd}(x)=\alpha^{\frac{n}{2}}$.

Proposition 4.11. Let $F$ be a field of characteristic 2 and let $(V, q)$ be a quadratic space over $F$. Let $\tau$ be an involution in $O(V, q)$ such that $J_{\tau}$ is of orthogonal type (in other words $\operatorname{dim} \operatorname{Fix}(V, \tau)=\frac{1}{2} \operatorname{dim} V$ ) on $C(V)$.

(a) If $\operatorname{dim} V=2$, then $\operatorname{disc} J_{\tau}=\theta(\tau)$.

(b) If $\operatorname{dim} V \geqslant 4$, then $\operatorname{disc} J_{\tau}=1 \in F^{\times} / F^{\times 2}$.

Proof. (a) Since $J_{\tau}$ is of orthogonal type, by (4.3), we have $\tau=\tau_{u}$ where $u \in V$ is an anisotropic vector. By (4.2), we have $u \in \operatorname{Alt}\left(C(V), J_{\tau_{u}}\right)$. On the other hand since $u^{2}=q(u) \neq 0$, the vector $u$ is invertible in $C(V)$ and by (4.10), $\operatorname{Nrd}(u)=q(u)$. So disc $J_{\tau_{u}}=\operatorname{Nrd}(u) F^{\times 2}=q(u) F^{\times 2}=\theta\left(\tau_{u}\right)$.

(b) By $(4.7),(V, \tau)$ has trivial maximal fixed orthogonal summand, so $(V, \tau)$ has a Wiitala decomposition $V=\mathbb{E}_{1} \perp \cdots \perp \mathbb{E}_{r} \perp \mathbb{A}_{1} \perp \cdots \perp \mathbb{A}_{s}$. If $\operatorname{dim} V=4$, then either $\tau=\tau_{1} \perp \tau_{2}$ where $\tau_{i}, i=1,2$, is a reflection of $\mathbb{E}_{i}$ or $\tau$ is an interchange isometry of $V$. In the first case we have $\left(C(V), J_{\tau}\right) \simeq\left(C\left(\mathbb{E}_{1}\right), J_{\tau_{1}}\right) \otimes$ $\left(C\left(\mathbb{E}_{2}\right), J_{\tau_{2}}\right)$. Using $[6,(7.3)]$, we have $\operatorname{disc} J_{\tau}=\operatorname{disc}\left(J_{\tau_{1}} \otimes J_{\tau_{2}}\right)=1$. Now consider the case where $\tau$ is an interchange isometry. Let $\{w, x, y, z\}$ be the basis of $V$ stated in (3.4). Then $J_{\tau}(x z)=\tau(z) \tau(x)=(w+z) x=w x+z x=$ $w x+x z+1$, i.e., $w x+1 \in \operatorname{Alt}\left(C(V), J_{\tau}\right)$. Since $B(x, w)=0$ we have $w x=x w$. We therefore obtain $(w x+1)^{2}=w x w x+1=w^{2} x^{2}+1=q(w) q(x)+1=1$. So $w x+1 \in C(V)^{*}$ and by $(4.10), \operatorname{Nrd}(w x+1)=1$. So $\operatorname{disc} J_{\tau}=\operatorname{Nrd}(w x+1) F^{\times 2}=$ $1 \in F^{\times} / F^{\times 2}$.

If $\operatorname{dim} V>4$, then using the Wiitala decomposition of $(V, \tau)$, there exist two nonzero subspaces $V_{1}$ and $V_{2}$ of $V$ such that $V=V_{1} \perp V_{2}$ and $V_{1}$ and $V_{2}$ are stable under $\tau$. So $\left(C(V), J_{\tau}\right) \simeq\left(C\left(V_{1}\right), J_{\tau_{1}}\right) \otimes\left(C\left(V_{2}\right), J_{\tau_{2}}\right)$, where $\tau_{i}=\left.\tau\right|_{V_{i}}$, $i=1,2$, and by $[6,(7.3)]$, $\operatorname{disc} J_{\tau}=\operatorname{disc}\left(J_{\tau_{1}} \otimes J_{\tau_{2}}\right)=1$. 
Lemma 4.12. Let $(V, q)$ be a quadratic space over a field $F$ of characteristic 2 and let $\tau$ be an involution in $O(V, q)$. Let $\left\{u_{1}, v_{1}, \cdots, u_{t}, v_{t}, u_{1}^{\prime}, v_{1}^{\prime}, \cdots, u_{r}^{\prime}, v_{r}^{\prime}\right.$, $\left.u_{1}^{\prime \prime}, v_{1}^{\prime \prime}, \cdots, u_{2 s}^{\prime \prime}, v_{2 s}^{\prime \prime}\right\}$ be a $\tau$-symplectic basis of $V$. Set $z=u_{1} v_{1}+\cdots+u_{t} v_{t}+$ $u_{1}^{\prime} v_{1}^{\prime}+\cdots+u_{r}^{\prime} v_{r}^{\prime}+u_{1}^{\prime \prime} v_{1}^{\prime \prime}+\cdots+u_{2 s}^{\prime \prime} v_{2 s}^{\prime \prime} \in C_{0}(V)$ and $\delta=q\left(u_{1}\right) q\left(v_{1}\right)+\cdots+$ $q\left(u_{t}\right) q\left(v_{t}\right)+q\left(u_{1}^{\prime}\right) q\left(v_{1}^{\prime}\right)+\cdots+q\left(u_{r}^{\prime}\right) q\left(v_{r}^{\prime}\right)+q\left(u_{1}^{\prime \prime}\right) q\left(v_{1}^{\prime \prime}\right)+\cdots+q\left(u_{2 s}^{\prime \prime}\right) q\left(v_{2 s}^{\prime \prime}\right) \in F$. Then $z^{2}=z+\delta \cdot 1$ and $J_{\tau}(z)=z+t \cdot 1$. (Note that according to (3.19), $\left.t=\operatorname{dim} \operatorname{Fix}(V, \tau)-\frac{1}{2} \operatorname{dim} V\right)$.

Proof. By [4, p. 123], we have $z^{2}=z+\delta \cdot 1$. Let $V=W \perp \mathbb{E}_{1} \perp \cdots \perp \mathbb{E}_{r} \perp \mathbb{A}_{1} \perp$ $\cdots \perp \mathbb{A}_{s}$ be a Wiitala decomposition of $(V, \tau)$ such that $\left\{u_{1}, v_{1}, \cdots, u_{t}, v_{t}, u_{1}^{\prime}, v_{1}^{\prime}\right.$ $\left., \cdots, u_{r}^{\prime}, v_{r}^{\prime}, u_{1}^{\prime \prime}, v_{1}^{\prime \prime}, \cdots, u_{2 s}^{\prime \prime}, v_{2 s}^{\prime \prime}\right\}$ is a $\tau$-symplectic basis with respect to this decomposition and $t=\operatorname{dim} \operatorname{Fix}(V, \tau)-\frac{1}{2} \operatorname{dim} V$. For $1 \leqslant i \leqslant t$, we have $J_{\tau}\left(u_{i} v_{i}\right)=\tau\left(v_{i}\right) \tau\left(u_{i}\right)=v_{i} u_{i}=u_{i} v_{i}+1$. Similarly for $1 \leqslant i \leqslant r$, we have $J_{\tau}\left(u_{i}^{\prime} v_{i}^{\prime}\right)=\tau\left(v_{i}^{\prime}\right) \tau\left(u_{i}^{\prime}\right)=\left(v_{i}^{\prime}+\frac{1}{q\left(u_{i}^{\prime}\right)} u_{i}^{\prime}\right) u_{i}^{\prime}=v_{i}^{\prime} u_{i}^{\prime}+1=u_{i}^{\prime} v_{i}^{\prime}$. Finally for $1 \leqslant i \leqslant s$, we have $J_{\tau}\left(u_{2 i-1}^{\prime \prime} v_{2 i-1}^{\prime \prime}+u_{2 i}^{\prime \prime} v_{2 i}^{\prime \prime}\right)=v_{2 i}^{\prime \prime} u_{2 i}^{\prime \prime}+v_{2 i-1}^{\prime \prime} u_{2 i-1}^{\prime \prime}=u_{2 i-1}^{\prime \prime} v_{2 i-1}^{\prime \prime}+u_{2 i}^{\prime \prime} v_{2 i}^{\prime \prime}$. We therefore obtain $J_{\tau}(z)=z+t \cdot 1$ and the result is proved.

Theorem 4.13. Let $(V, q)$ be a quadratic space over a field $F$ of characteristic 2 and let $\tau$ be an involution in $O(V, q)$. Let $t=\operatorname{dim} \operatorname{Fix}(V, \tau)-\frac{1}{2} \operatorname{dim} V$. Suppose that $\Delta(q)$ is nontrivial. Consider the involution $J_{\tau}$ on $C_{0}(V)$ :

(a) If $t \equiv 0 \bmod 2$ then $J_{\tau}$ is of the first kind. Furthermore if $t=0$ then $J_{\tau}$ is of orthogonal type and if $t \neq 0$ then $J_{\tau}$ is of symplectic type.

(b) If $t \equiv 1 \bmod 2$ then $J_{\tau}$ is of the second kind.

Proof. Let $\left\{u_{1}, v_{1}, \cdots, u_{n}, v_{n}\right\}$ be a $\tau$-symplectic basis of $V$ and let $W$ be a maximal fixed orthogonal summand of $(V, \tau)$. Set $z=u_{1} v_{1}+\cdots+u_{n} v_{n}$. Then by (4.12), we have $J_{\tau}(z)=t \cdot 1+z$ where $t=\frac{1}{2} \operatorname{dim} W=\operatorname{dim} \operatorname{Fix}(V, \tau)-\frac{1}{2} \operatorname{dim} V$. By $[4,(13.12)], Z\left(C_{0}(V)\right)$ is generated as an $F$-algebra by 1 and $z$ over $F$. If $t \equiv 0 \bmod 2$ then $J_{\tau}(z)=z$, i.e., $\left.J_{\tau}\right|_{Z\left(C_{0}(V)\right)}=$ id. Hence $J_{\tau}$ is of the first kind. Now if $t=0$, then $W=0$ and by $(4.7), J_{\tau}$ is of orthogonal type on $C(V)$, so by $[6,(2.6)] 1 \notin \operatorname{Alt}\left(C(V), J_{\tau}\right)$. So $1 \notin \operatorname{Alt}\left(C_{0}(V), J_{\tau}\right)$ and by $[6,(2.6)], J_{\tau}$ is of orthogonal type on $C_{0}(V)$. Similarly if $t \neq 0$, then $t \geqslant 2$, so $u_{1}, v_{1} \in W$ and $J_{\tau}\left(u_{1} v_{1}\right)=v_{1} u_{1}=u_{1} v_{1}+1$. Hence $1 \in \operatorname{Alt}\left(C_{0}(V), J_{\tau}\right)$ and again by $[6,(2.6)]$, $J_{\tau}$ is of symplectic type.

If $t \equiv 1 \bmod 2$ then $J_{\tau}(z)=z+1$, so $J_{\tau}$ is of the second kind.

Remark 4.14. Let $(V, q)$ be a quadratic space over a field $F$ of characteristic 2 and let $\tau$ be an involution in $O(V, q)$. If $\Delta(q)$ is nontrivial, then $C_{0}(V)$ is a central simple algebra over $Z=Z\left(C_{0}(V)\right)$ and $Z / F$ is a quadratic field extension. More precisely if $\left\{u_{1}, v_{1}, \cdots, u_{n}, v_{n}\right\}$ is a symplectic basis of $V$ and $z=u_{1} v_{1}+\cdots+u_{n} v_{n}$, then $Z=F(z)$. If $x=\alpha+\beta z \in Z$ for $\alpha, \beta \in F$ and $x^{2} \in F$, then by (4.12) we have $\alpha^{2}+\beta^{2}(z+\delta) \in F$ where $\delta=\sum_{i=1}^{n} q\left(u_{i}\right) q\left(v_{i}\right)$. So $\beta=0$ and $x \in F$. This shows that $Z^{\times 2} \cap F^{\times}=F^{\times 2}$. Therefore, there exists a natural injection $F^{\times} / F^{\times 2} \hookrightarrow Z^{\times} / Z^{\times 2}$ and we may consider $F^{\times} / F^{\times 2}$ as a subgroup of $Z^{\times} / Z^{\times 2}$.

Proposition 4.15. Let $(V, q)$ be a quadratic space over a field $F$ of characteristic 2 and let $\tau$ be an involution in $O(V, q)$. Suppose that $\Delta(q)$ is nontrivial and $J_{\tau}$ is an involution of orthogonal type (in other words $\operatorname{dim} \operatorname{Fix}(V, \tau)=\frac{1}{2} \operatorname{dim} V$ ) on $C_{0}(V)$. Let $i: F^{\times} / F^{\times 2} \hookrightarrow Z^{\times} / Z^{\times 2}$ be the natural injection discussed in (4.14). 
(a) If $\operatorname{dim} V=4$, then $\operatorname{disc} J_{\tau}=i(\theta(\tau))$.

(b) If $\operatorname{dim} V \geqslant 6$, then $\operatorname{disc} J_{\tau}$ is trivial.

Proof. Since $\Delta(q)$ is nontrivial, by $[4,(13.12)], C_{0}(V)$ is a central simple algebra. Let $\left\{u_{1}, v_{1}, \cdots, u_{n}, v_{n}\right\}$ be a $\tau$-symplectic basis of $V$. By $(3.19),(V, \tau)$ has trivial maximal fixed orthogonal summand, so $(V, \tau)$ has a Wiitala decomposition $V=$ $\mathbb{E}_{1} \perp \cdots \perp \mathbb{E}_{r} \perp \mathbb{A}_{1} \perp \cdots \perp \mathbb{A}_{s}$. If $\tau$ is of interchanging kind, then by (3.20), $\Delta(q)$ is trivial which contradicts the assumption. So $\tau$ is of reflectional kind and we may assume that $\tau$ is an orthogonal sum of reflections $\tau_{i}=\tau_{u_{i}}$ of $\mathbb{E}_{i}$. We have $J_{\tau}\left(u_{1} v_{2}\right)=\tau_{2}\left(v_{2}\right) \tau_{1}\left(u_{1}\right)=\left(v_{2}+\frac{1}{q\left(u_{2}\right)} u_{2}\right) u_{1}=v_{2} u_{1}+\frac{1}{q\left(u_{2}\right)} u_{2} u_{1}=u_{1} v_{2}+$ $\frac{1}{q\left(u_{2}\right)} u_{1} u_{2}$, i.e., $u_{1} u_{2} \in \operatorname{Alt}\left(C_{0}(V), J_{\tau}\right)$. We also have $\left(u_{1} u_{2}\right)^{2}=q\left(u_{1}\right) q\left(u_{2}\right) \neq 0$, so $u_{1} u_{2} \in C_{0}(V)^{*}$.

(a) By (4.10), $\operatorname{Nrd}\left(u_{1} u_{2}\right)=q\left(u_{1}\right) q\left(u_{2}\right)$. Therefore, $\operatorname{disc} J_{\tau}=\operatorname{Nrd}\left(u_{1} u_{2}\right) Z^{\times 2}=$ $q\left(u_{1}\right) q\left(u_{2}\right) Z^{\times 2}$. Finally note that we have $\tau=\tau_{u_{1}} \tau_{u_{2}}$ where we consider $\tau_{u_{i}}$ as a reflection of $V$ and therefore, $\operatorname{disc} J_{\tau}=i(\theta(\tau))=q\left(u_{1}\right) q\left(u_{2}\right) Z^{\times 2}$.

(b) As $\operatorname{dim} V \geqslant 6$, we obtain $\operatorname{deg}\left(C_{0}(V)\right) \equiv 0 \bmod 4$. Since $\left(u_{1} u_{2}\right)^{2}=$ $q\left(u_{1}\right) q\left(u_{2}\right)$, by $(4.10)$, we have $\operatorname{Nrd}\left(u_{1} u_{2}\right)=1$, so $\operatorname{disc} J_{\tau}=\operatorname{Nrd}\left(u_{1} u_{2}\right) Z^{\times 2}$ is trivial.

Remark 4.16. The assumption that $\Delta(q)$ is nontrivial in (4.15) is necessary, because by $[4,(13.12)]$, if $\Delta(q)$ is trivial, then $C_{0}(V)$ is not central simple algebra and the reduced norm is not defined. Also we have assumed that $\operatorname{dim} V \geqslant 4$, because if $\operatorname{dim} V=2$ then $J_{\tau}$ is identity and $\operatorname{disc} J_{\tau}$ is not defined.

\section{On a result of Mammone, Tignol and Wads- worth}

In $[10]$ it has been shown that if $(\mathbb{E}, \varphi)$ is the quadratic plane $[a, b]$ where $a \neq 0$, then for any quadratic space $(V, q)$ we have $C(\mathbb{E} \perp V, \varphi \perp q) \simeq C\left(\mathbb{E}^{\prime}, \varphi^{\prime}\right) \otimes$ $C(V, a \cdot q)$ and $C_{0}(\mathbb{E} \perp V, \varphi \perp q) \simeq C_{0}\left(\mathbb{E}^{\prime \prime}, \varphi^{\prime \prime}\right) \otimes C(V, a \cdot q)$, where $\left(\mathbb{E}^{\prime}, \varphi^{\prime}\right)$ and $\left(\mathbb{E}^{\prime \prime}, \varphi^{\prime \prime}\right)$ are quadratic planes $\left[a, b+a^{-1} \delta_{1}\right]$ and $\left[1, \delta_{1}+\delta_{2}\right]$, respectively, here $\delta_{1} \in F$ is a representative of the class $\Delta(q) \in F / \wp(F)$ and $\delta_{2} \in F$ is a representative of the class $\Delta(\varphi) \in F / \wp(F)$, i.e., $\Delta(q)=\delta_{1}+\wp(F) \in F / \wp(F)$ and $\Delta(\varphi)=\delta_{2}+\wp(F) \in F / \wp(F)$. We complement these results by showing that:

Proposition 5.1. Let $F$ be a field of characteristic 2 and let $(\mathbb{E}, \varphi)$ and $(V, q)$ be two quadratic spaces over $F$ such that $\operatorname{dim} \mathbb{E}=2$.

(i) If $\varphi \simeq[a, b]$ for $a, b \in F$ and $a \neq 0$, then $C(\mathbb{E} \perp V, \varphi \perp q) \simeq C\left(\mathbb{E}^{\prime}, \varphi^{\prime}\right) \otimes$ $C(V, a \cdot q) \simeq C\left(\mathbb{E}^{\prime} \perp V, \varphi^{\prime} \perp a \cdot q\right)$ where $\left(\mathbb{E}^{\prime}, \varphi^{\prime}\right)$ is the quadratic plane $\left[a, b+a^{-1} \delta\right]$ and $\delta \in F$ is a representative of the class $\Delta(q) \in F / \wp(F)$. ([10, Prop. 5])

(ii) Let $\sigma$ and $\tau$ be involutions in $O(\mathbb{E}, \varphi)$ and $O(V, q)$, respectively. Then there exist $a, b \in F$ with $a \neq 0$ such that $\varphi \simeq[a, b]$ and $\left(C(\mathbb{E} \perp V, \varphi \perp q), J_{\sigma \perp \tau}^{\varphi \perp q}\right) \simeq$ $\left(C\left(\mathbb{E}^{\prime} \perp V, \varphi^{\prime} \perp a \cdot q\right), J_{\sigma^{\prime} \perp \tau}^{\varphi^{\prime} \perp a \cdot q}\right)$ where $\left(\mathbb{E}^{\prime}, \varphi^{\prime}\right)$ is the quadratic plane $\left[a, b+a^{-1} \delta\right]$, $\delta \in F$ is a representative of the class $\Delta(q) \in F / \wp(F)$ and $\sigma^{\prime}$ is a suitable involution in $O\left(\mathbb{E}^{\prime}, \varphi^{\prime}\right)$. More precisely suppose that $\{x, y\}$ is a standard symplectic basis of $\left[a, b+a^{-1} \delta\right]$ and $t=\operatorname{dim} \operatorname{Fix}(\mathbb{E} \perp V, \sigma \perp \tau)-\frac{1}{2} \operatorname{dim}(\mathbb{E} \perp V)$. If $t \equiv 0 \bmod 2$ then $\sigma^{\prime}=\tau_{x}$ and if $t \equiv 1 \bmod 2$ then $\sigma^{\prime}=\mathrm{id}_{\mathbb{E}^{\prime}}$. In other words $\sigma^{\prime}(x)=x$ and $\sigma^{\prime}(y)=y+(t+1) x^{-1}$. 
Proof. (i) This part has been proved in [10, Prop. 5], but as in the proof of part $(i i)$ we need an explicit form of this isomorphism, we recall its construction here. Let $\{u, v\}$ be a standard symplectic basis of $(\mathbb{E}, \varphi=[a, b])$, i.e., $\varphi(u)=a$, $\varphi(v)=b$ and $B_{\varphi}(u, v)=1$. Let $\left\{u_{1}, v_{1}, \cdots, u_{n}, v_{n}\right\}$ be a symplectic basis of $(V, q)$ and set $\delta=\sum_{i=1}^{n} q\left(u_{i}\right) q\left(v_{i}\right) \in F$. Let $\{x, y\}$ be a standard symplectic basis of $\left(\mathbb{E}^{\prime}, \varphi^{\prime}=\left[a, b+a^{-1} \delta\right]\right)$. Define the linear map $f:(\mathbb{E} \perp V, \varphi \perp q) \rightarrow$ $C\left(\mathbb{E}^{\prime} \perp V, \varphi^{\prime} \perp a \cdot q\right)$ via $f(u)=x, f(v)=y+a^{-1} x^{-1}\left(u_{1} v_{1}+\cdots+u_{n} v_{n}\right)$, $f\left(u_{i}\right)=x^{-1} u_{i}$ and $f\left(v_{i}\right)=x^{-1} v_{i}, 1 \leqslant i \leqslant n$. Let $B=B_{\varphi \perp q}$ be the bilinear form associated to the quadratic form $\varphi \perp q$. We have the following relations:

$$
\begin{aligned}
f(u)^{2} & =x^{2}=\varphi^{\prime}(x)=a=\varphi(u), \\
f\left(u_{i}\right)^{2} & =\left(x^{-1} u_{i}\right)^{2}=x^{-2} u_{i}^{2}=\left(a^{-1}\right)\left(a \cdot q\left(u_{i}\right)\right)=q\left(u_{i}\right), \\
f\left(v_{i}\right)^{2} & =\left(x^{-1} v_{i}\right)^{2}=x^{-2} v_{i}^{2}=\left(a^{-1}\right)\left(a \cdot q\left(v_{i}\right)\right)=q\left(v_{i}\right) .
\end{aligned}
$$

Since $\left\{u_{1}, v_{1}, \cdots, u_{n}, v_{n}\right\}$ is a symplectic basis of $(V, q)$, the set $\left\{u_{1}, a^{-1} v_{1}\right.$, $\left.\cdots, u_{n}, a^{-1} v_{n}\right\}$ is also a symplectic basis of $(V, a \cdot q)$. We have $a \cdot q\left(u_{i}\right)=a q\left(u_{i}\right)$ and $a \cdot q\left(a^{-1} v_{i}\right)=a^{-1} q\left(v_{i}\right)$. So by (4.12) we have (the calculations are performed in $C(V, a \cdot q))$ :

$$
\begin{aligned}
\left(a^{-1} u_{1} v_{1}+\cdots+a^{-1} u_{n} v_{n}\right)^{2}= & \left(a^{-1} u_{1} v_{1}+\cdots+a^{-1} u_{n} v_{n}\right) \\
& +a q\left(u_{1}\right) a^{-1} q\left(v_{1}\right)+\cdots+a q\left(u_{n}\right) a^{-1} q\left(v_{n}\right) \\
= & a^{-1}\left(u_{1} v_{1}+\cdots+u_{n} v_{n}\right)+\delta,
\end{aligned}
$$

So $\left(u_{1} v_{1}+\cdots+u_{n} v_{n}\right)^{2}=a\left(u_{1} v_{1}+\cdots+u_{n} v_{n}\right)+a^{2} \delta$ in $C(V, a \cdot q)$ and we obtain:

$$
\begin{aligned}
f(v)^{2}= & \left(y+a^{-1} x^{-1}\left(u_{1} v_{1}+\cdots+u_{n} v_{n}\right)\right)^{2} \\
= & \varphi^{\prime}(y)+a^{-2} x^{-2}\left(a\left(u_{1} v_{1}+\cdots+u_{n} v_{n}\right)+a^{2} \delta\right) \\
& +a^{-1}\left(x^{-1} y+y x^{-1}\right)\left(u_{1} v_{1}+\cdots+u_{n} v_{n}\right) \\
= & \left(b+a^{-1} \delta\right)+a^{-3}\left(a\left(u_{1} v_{1}+\cdots+u_{n} v_{n}\right)+a^{2} \delta\right) \\
& +a^{-2}(x y+y x)\left(u_{1} v_{1}+\cdots+u_{n} v_{n}\right) \\
= & b+a^{-1} \delta+a^{-2}\left(u_{1} v_{1}+\cdots+u_{n} v_{n}\right)+a^{-1} \delta \\
& +a^{-2}\left(u_{1} v_{1}+\cdots+u_{n} v_{n}\right)=b=\varphi(v) .
\end{aligned}
$$

Now clearly for every $1 \leqslant i \leqslant n$, we have $f(u) f\left(u_{i}\right)+f\left(u_{i}\right) f(u)=0=B\left(u, u_{i}\right)$ and $f(u) f\left(v_{i}\right)+f\left(v_{i}\right) f(u)=0=B\left(u, v_{i}\right)$. Similarly for every $1 \leqslant i \neq j \leqslant n$, we have $f\left(u_{i}\right) f\left(u_{j}\right)+f\left(u_{j}\right) f\left(u_{i}\right)=0=B\left(u_{i}, u_{j}\right), f\left(v_{i}\right) f\left(v_{j}\right)+f\left(v_{j}\right) f\left(v_{i}\right)=0=$ $B\left(v_{i}, v_{j}\right)$ and $f\left(u_{i}\right) f\left(v_{j}\right)+f\left(v_{j}\right) f\left(u_{i}\right)=0=B\left(u_{i}, v_{j}\right)$. For every $1 \leqslant i \leqslant n$, we have

$$
\begin{aligned}
f\left(u_{i}\right) f\left(v_{i}\right)+f\left(v_{i}\right) f\left(u_{i}\right) & =x^{-1} u_{i} x^{-1} v_{i}+x^{-1} v_{i} x^{-1} u_{i}=x^{-2}\left(u_{i} v_{i}+v_{i} u_{i}\right) \\
& =a^{-1}\left(a \cdot B_{q}\left(u_{i}, v_{i}\right)\right)=B\left(u_{i}, v_{i}\right) .
\end{aligned}
$$

We also have

$$
\begin{aligned}
f(u) f(v)+f(v) f(u)= & x\left(y+a^{-1} x^{-1}\left(u_{1} v_{1}+\cdots+u_{n} v_{n}\right)\right) \\
& +\left(y+a^{-1} x^{-1}\left(u_{1} v_{1}+\cdots+u_{n} v_{n}\right)\right) x \\
= & x y+y x=1=B(u, v) .
\end{aligned}
$$


For every $1 \leqslant i \leqslant n$, we have

$$
\begin{aligned}
f\left(u_{i}\right) f(v)+f(v) f\left(u_{i}\right)= & x^{-1} u_{i}\left(y+a^{-1} x^{-1}\left(u_{1} v_{1}+\cdots+u_{n} v_{n}\right)\right) \\
& +\left(y+a^{-1} x^{-1}\left(u_{1} v_{1}+\cdots+u_{n} v_{n}\right)\right) x^{-1} u_{i} \\
= & x^{-1} u_{i} y+y x^{-1} u_{i} \\
& +x^{-1} u_{i}\left(a^{-1} x^{-1} u_{i} v_{i}\right)+\left(a^{-1} x^{-1} u_{i} v_{i}\right) x^{-1} u_{i} \\
= & \left(a^{-1} x\right) u_{i} y+y\left(a^{-1} x\right) u_{i} \\
& +a^{-1} x^{-2} u_{i}\left(u_{i} v_{i}\right)+a^{-1} x^{-2}\left(u_{i} v_{i}\right) u_{i} \\
= & a^{-1} u_{i}(x y+y x)+a^{-2}\left(u_{i} u_{i} v_{i}+u_{i}\left(u_{i} v_{i}+a\right)\right) \\
= & a^{-1} u_{i}+a^{-1} u_{i}=0=B\left(u_{i}, v\right) .
\end{aligned}
$$

Similarly for every $1 \leqslant i \leqslant n$, we have

$$
f\left(v_{i}\right) f(v)+f(v) f\left(v_{i}\right)=B\left(v_{i}, v\right) .
$$

So the map $f$ is compatible with $\varphi \perp q$ and it can be extended to an isomorphism $f: C(\mathbb{E} \perp V, \varphi \perp q) \rightarrow C\left(\mathbb{E}^{\prime} \perp V, \varphi^{\prime} \perp a \cdot q\right)$.

(ii) By (3.12), there is an anisotropic vector $u \in \mathbb{E}$ such that $\sigma(u)=u$. (So if $\sigma \neq \operatorname{id}_{\mathbb{E}}$, then $\sigma=\tau_{u}$ ). Extend $\{u\}$ to a symplectic basis $\{u, v\}$ of $\mathbb{E}$. Set $a=\varphi(u)$ and $b=\varphi(v)$. Then $\varphi \simeq[a, b]$. By $(i)$, we have $C(\mathbb{E} \perp V, \varphi \perp$ $q) \simeq C\left(\mathbb{E}^{\prime} \perp V, \varphi^{\prime} \perp a \cdot q\right)$ where $\left(\mathbb{E}^{\prime}, \varphi^{\prime}\right)$ is the quadratic plane $\left[a, b+a^{-1} \delta\right]$ and $\delta \in F$ is a representative of the class $\Delta(q) \in F / \wp(F)$. We claim that $\left(C(\mathbb{E} \perp V, \varphi \perp q), J_{\sigma \perp \tau}^{\varphi \perp q}\right) \simeq\left(C\left(\mathbb{E}^{\prime} \perp V, \varphi^{\prime} \perp a \cdot q\right), J_{\sigma^{\prime} \perp \tau}^{\varphi^{\prime} \perp a \cdot q}\right)$. We must show that for every $w \in C(\mathbb{E} \perp V)$ we have $\left(f \circ J_{\sigma \perp \tau}^{\varphi \perp q}\right)(w)=\left(J_{\sigma^{\prime} \perp \tau}^{\varphi^{\prime} \perp a \cdot q} \circ f\right)(w)$. It is enough to check this for $w=u, v, u_{i}, v_{i}, i=1, \cdots, n$. Note that in both cases $\sigma^{\prime}=\operatorname{id}_{\mathbb{E}^{\prime}}$ and $\sigma^{\prime}=\tau_{x}$ we have $\sigma^{\prime}(x)=x$. We have

$$
\begin{aligned}
\left(f \circ J_{\sigma \perp \tau}^{\varphi \perp q}\right)(u) & =f(u)=x=\sigma^{\prime}(x)=J_{\sigma^{\prime} \perp \tau}^{\varphi^{\prime} \perp a \cdot q}(x) \\
& =\left(J_{\sigma^{\prime} \perp \tau}^{\varphi^{\prime} \perp a \cdot q} \circ f\right)(u) .
\end{aligned}
$$

Since for every $1 \leqslant i \leqslant n, \tau\left(u_{i}\right) \in V$, we get $f\left(\tau\left(u_{i}\right)\right)=x^{-1} \tau\left(u_{i}\right)$ and similarly $f\left(\tau\left(v_{i}\right)\right)=x^{-1} \tau\left(v_{i}\right)$. So for every $1 \leqslant i \leqslant n$ we have

$$
\begin{aligned}
\left(f \circ J_{\sigma \perp \tau}^{\varphi \perp q}\right)\left(u_{i}\right) & =f\left(\tau\left(u_{i}\right)\right)=x^{-1} \tau\left(u_{i}\right)=\sigma^{\prime}\left(x^{-1}\right) \tau\left(u_{i}\right) \\
& =J_{\sigma^{\prime} \perp \tau}^{\varphi^{\prime} \perp a \cdot q}\left(x^{-1} u_{i}\right)=\left(J_{\sigma^{\prime} \perp \tau}^{\varphi^{\prime} \perp a \cdot q} \circ f\right)\left(u_{i}\right) .
\end{aligned}
$$

Similarly for every $1 \leqslant i \leqslant n$ we have

$$
\begin{aligned}
\left(f \circ J_{\sigma \perp \tau}^{\varphi \perp q}\right)\left(v_{i}\right) & =f\left(\tau\left(v_{i}\right)\right)=x^{-1} \tau\left(v_{i}\right)=\sigma^{\prime}\left(x^{-1}\right) \tau\left(v_{i}\right) \\
& =J_{\sigma^{\prime} \perp \tau}^{\varphi^{\prime} \perp a \cdot q}\left(x^{-1} v_{i}\right)=\left(J_{\sigma^{\prime} \perp \tau}^{\varphi^{\prime} \perp a \cdot q} \circ f\right)\left(v_{i}\right) .
\end{aligned}
$$

Set $t_{1}=\operatorname{dim} \operatorname{Fix}(\mathbb{E}, \sigma)-\frac{1}{2} \operatorname{dim} \mathbb{E}$ and $t_{2}=\operatorname{dim} \operatorname{Fix}(V, \tau)-\frac{1}{2} \operatorname{dim} V$. Then $t=t_{1}+t_{2}$. Note that $\tau$ is also an involution in $O\left(V, a \cdot q, a \cdot B_{q}\right)$. Since $\left\{u_{1}, v_{1}, \cdots\right.$, $\left.u_{n}, v_{n}\right\}$ is a $\tau$-symplectic basis of $(V, q)$, the set $\left\{u_{1}, a^{-1} v_{1}, \cdots, u_{n}, a^{-1} v_{n}\right\}$ is also a $\tau$-symplectic basis of $(V, a \cdot q)$. By (4.12), we have $J_{\tau}^{a \cdot q}\left(u_{1} a^{-1} v_{1}+\cdots+\right.$ $\left.u_{n} a^{-1} v_{n}\right)=t_{2} \cdot 1+u_{1}\left(a^{-1} v_{1}\right)+\cdots+u_{n}\left(a^{-1} v_{n}\right)$ in $C(V, a \cdot q)$. So $\tau\left(v_{1}\right) \tau\left(u_{1}\right)+\cdots+$ $\tau\left(v_{n}\right) \tau\left(u_{n}\right)=t_{2} \cdot a+u_{1} v_{1}+\cdots+u_{n} v_{n}$ in $C(V, a \cdot q)$. We have $\sigma^{\prime}(y)=y+(t+1) x^{-1}$. 
So using (3.13) we obtain

$$
\begin{aligned}
\left(J_{\sigma^{\prime} \perp \tau}^{\varphi^{\prime} \perp a \cdot q} \circ f\right)(v) & =J_{\sigma^{\prime} \perp \tau}^{\varphi^{\prime} \perp a \cdot q}\left(y+a^{-1} x^{-1}\left(u_{1} v_{1}+\cdots+u_{n} v_{n}\right)\right) \\
& =\sigma^{\prime}(y)+a^{-1}\left(\tau\left(v_{1}\right) \tau\left(u_{1}\right)+\cdots+\tau\left(v_{n}\right) \tau\left(u_{n}\right)\right) \sigma^{\prime}\left(x^{-1}\right) \\
& =y+(t+1) x^{-1}+a^{-1}\left(t_{2} \cdot a+u_{1} v_{1}+\cdots+u_{n} v_{n}\right) x^{-1} \\
& =y+\left(t+t_{2}+1\right) x^{-1}+a^{-1}\left(u_{1} v_{1}+\cdots+u_{n} v_{n}\right) x^{-1} \\
& =y+a^{-1}\left(u_{1} v_{1}+\cdots+u_{n} v_{n}\right) x^{-1}+\left(t_{1}+1\right) x^{-1} \\
& =f\left(v+\left(t_{1}+1\right) u^{-1}\right)=f(\sigma(v))=\left(f \circ J_{\sigma \perp \tau}^{\varphi \perp q}\right)(v),
\end{aligned}
$$

which completes the proof.

Corollary 5.2. Let $(V, q)$ be a $2 n$-dimensional quadratic space over a field $F$ of characteristic 2 and let $\tau$ be a reflection of $V$. If $n \geqslant 2$, then there exists a $2 n$ dimensional quadratic space $\left(V^{\prime}, q^{\prime}\right)$ over $F$ such that $\left(C(V), J_{\tau}\right) \simeq\left(C\left(V^{\prime}\right), J_{\mathrm{id}}\right)$.

Proof. Let $u_{1} \in V$ be an anisotropic vector such that $\tau=\tau_{u_{1}}$. Extend $\left\{u_{1}\right\}$ to a symplectic basis $\left\{u_{1}, v_{1}, \cdots, u_{n}, v_{n}\right\}$ of $V$. Set $\mathbb{E}_{1}=F u_{1}+F v_{1}$ and $V_{1}=F u_{2}+$ $F v_{2}+\cdots+F u_{n}+F v_{n}$. Then $V=\mathbb{E}_{1} \perp V_{1},\left.\tau\right|_{\mathbb{E}_{1}}$ is a reflection and $\left.\tau\right|_{V_{1}}=$ id. Set $t:=\operatorname{dim} \operatorname{Fix}(V, \tau)-\frac{1}{2} \operatorname{dim} V=(2 n-1)-n=n-1$. If $n \equiv 0 \bmod 2$, then by $\left(5.1\right.$ (ii)), we have $\left(C(V), J_{\tau}\right) \simeq\left(C\left(\mathbb{E}_{1} \perp V_{1}\right), J_{\tau \mid \mathbb{E}_{1} \perp \text { id }}\right) \simeq\left(C\left(V^{\prime}\right), J_{\text {id }}\right)$ where $\left(V^{\prime}, q^{\prime}\right)$ is a $2 n$-dimensional quadratic space. If $n \equiv 1 \bmod 2$, we have $n \geqslant 3$, so $\operatorname{dim} V_{1} \geqslant 4$. As $\left(V_{1}, q\right)$ is regular we can write $V_{1}=\mathbb{E}_{2} \perp V_{2}$ where $\mathbb{E}_{2}$ is a quadratic plane. We have $\operatorname{dim} \operatorname{Fix}\left(\mathbb{E}_{1} \perp V_{2},\left.\tau\right|_{\mathbb{E}_{1}} \perp\right.$ id $)-\frac{1}{2} \operatorname{dim}\left(\mathbb{E}_{1} \perp V_{2}\right)=$ $(2 n-3)-(n-1)=n-2 \equiv 1 \bmod 2$. By (4.4) and (5.1 (ii)), we have

$$
\begin{aligned}
\left(C(V), J_{\tau}\right) & \simeq\left(C\left(\mathbb{E}_{1}\right), J_{\left.\tau\right|_{\mathbb{E}_{1}}}\right) \otimes\left(C\left(V_{1}\right), J_{\mathrm{id}}\right) \\
& \simeq\left(\left(C\left(\mathbb{E}_{1}\right), J_{\tau \mid \mathbb{\mathbb { E }}_{1}}\right) \otimes\left(C\left(V_{2}\right), J_{\mathrm{id}}\right)\right) \otimes\left(C\left(\mathbb{E}_{2}\right), J_{\mathrm{id}}\right) \\
& \simeq\left(C\left(V_{3}\right), J_{\mathrm{id}}\right) \otimes\left(C\left(\mathbb{E}_{2}\right), J_{\mathrm{id}}\right) \simeq\left(C\left(V^{\prime}\right), J_{\mathrm{id}}\right) .
\end{aligned}
$$

Here $V_{3}$ is a $(2 n-2)$-dimensional quadratic space which is determined by (5.1 (ii)) and $V^{\prime}=V_{3} \perp \mathbb{E}_{3}$.

Corollary 5.3. Let $F$ be a field of characteristic 2 and let $(\mathbb{E}, \varphi)$ and $(V, q)$ be quadratic spaces over $F$ such that $\operatorname{dim} \mathbb{E}=2$. Let $\left(\mathbb{E}^{\prime}, \varphi^{\prime}\right)$ be the quadratic plane $\left[1, \delta+\delta^{\prime}\right]$ where $\delta \in F$ is a representative of the class $\Delta(\varphi) \in F / \wp(F)$ and $\delta^{\prime} \in F$ is a representative of the class $\Delta(q) \in F / \wp(F)$. Let $\sigma$ and $\tau$ be involutions in $O(\mathbb{E}, \varphi)$ and $O(V, q)$, respectively.

(i) We have $C_{0}(\mathbb{E} \perp V, \varphi \perp q) \simeq C_{0}\left(\mathbb{E}^{\prime}, \varphi^{\prime}\right) \otimes C(V, a \cdot q)$. ([10, Prop. 5])

(ii) We have $\left(C_{0}(\mathbb{E}), J_{\sigma}\right) \otimes\left(C(V), J_{\tau}\right) \simeq\left(C_{0}\left(\mathbb{E}^{\prime} \perp V\right), J_{\sigma^{\prime} \perp \tau}\right)$ where $\sigma^{\prime}$ is a suitable involution in $O\left(\mathbb{E}^{\prime}, \varphi^{\prime}\right)$.

(iii) There exists an element $a \in D_{F}(\varphi)$ such that $\left(C_{0}(\mathbb{E} \perp V), J_{\sigma \perp \tau}\right) \simeq$ $\left(C_{0}\left(\mathbb{E}^{\prime}\right), J_{\sigma^{\prime}}\right) \otimes\left(C(V, a \cdot q), J_{\tau}^{a \cdot q}\right)$ where $\sigma^{\prime}$ is a suitable involution in $O\left(\mathbb{E}^{\prime}, \varphi^{\prime}\right)$.

More precisely if $\{x, y\}$ is a standard symplectic basis of $\left[1, \delta+\delta^{\prime}\right]$ and $t=$ $\operatorname{dim} \operatorname{Fix}(\mathbb{E} \perp V, \sigma \perp \tau)-\frac{1}{2} \operatorname{dim}(\mathbb{E} \perp V)$, the involution $\sigma^{\prime} \in O\left(\mathbb{E}^{\prime}, \varphi^{\prime}\right)$ in (ii) and (iii) can be described as follows: if $t \equiv 0 \bmod 2$ then $\sigma^{\prime}=\tau_{x}$ and if $t \equiv 1$ $\bmod 2$ then $\sigma^{\prime}=\operatorname{id}_{\mathbb{E}^{\prime}}$.

Proof. By (3.12), there is an anisotropic vector $u \in \mathbb{E}$ such that $\sigma(u)=u$ (so if $\sigma \neq \operatorname{id}_{\mathbb{E}}$, then $\left.\sigma=\tau_{u}\right)$. Extend $\{u\}$ to a symplectic basis $\{u, v\}$ of $\mathbb{E}$. Set $a=$ 
$\varphi(u)$ and $b=\varphi(v)$. Then $\varphi \simeq[a, b]$. Let $\left\{u_{1}, v_{1}, \cdots, u_{n}, v_{n}\right\}$ be a $\tau$-symplectic basis of $V$. Let $\delta=\varphi(u) \varphi(v)=a b \in F$ and $\delta^{\prime}=\sum_{i=1}^{n} q\left(u_{i}\right) q\left(v_{i}\right) \in F$.

(ii) $\operatorname{By}(4.5),\left(C_{0}(\mathbb{E}, \varphi), J_{\sigma}\right) \simeq\left(C_{0}(\mathbb{E}, a \cdot \varphi), J_{\sigma}\right)$, so we may assume that $a=1$, $b=\delta$ and $\varphi=[1, \delta]$. By (5.1 (ii)), there exists an isomorphism $(C(\mathbb{E} \perp V, \varphi \perp$ q), $\left.J_{\sigma \perp \tau}\right) \simeq\left(C\left(\mathbb{E}^{\prime} \perp V, \varphi^{\prime} \perp q\right), J_{\sigma^{\prime} \perp \tau}\right)$, such that if $t \equiv 0 \bmod 2$ then $\sigma^{\prime}=\tau_{x}$ and if $t \equiv 1 \bmod 2$ then $\sigma^{\prime}=\mathrm{id}_{\mathbb{E}^{\prime}}$. So $\sigma^{\prime}(x)=x$ and if $\sigma^{\prime} \neq \mathrm{id}$, then $\sigma^{\prime}=\tau_{x}$. Using this isomorphism and (4.4), the map $\psi:\left(C(\mathbb{E}, \varphi), J_{\sigma}\right) \otimes\left(C(V, q), J_{\tau}\right) \simeq$ $\left(C(\mathbb{E} \perp V, \varphi \perp q), J_{\sigma \perp \tau}\right) \simeq\left(C\left(\mathbb{E}^{\prime} \perp V\right), J_{\sigma^{\prime} \perp \tau}\right)$ induced by $\psi(u \otimes 1)=x$, $\psi(v \otimes 1)=y+x^{-1}\left(u_{1} v_{1}+\cdots+u_{n} v_{n}\right), \psi\left(1 \otimes u_{i}\right)=x^{-1} u_{i}$ and $\psi\left(1 \otimes v_{i}\right)=$ $x^{-1} v_{i}$ is an isomorphism. The restriction of $\psi$ to $\left(C_{0}(\mathbb{E}), J_{\sigma}\right) \otimes\left(C(V), J_{\tau}\right)$ maps $\left(C_{0}(\mathbb{E}), J_{\sigma}\right) \otimes\left(C(V), J_{\tau}\right)$ to $\left(C_{0}\left(\mathbb{E}^{\prime} \perp V\right), J_{\sigma^{\prime} \perp \tau}\right)$ and the result is proved.

(iii) By (4.5), we have

$$
\left(C_{0}(\mathbb{E} \perp V), J_{\sigma \perp \tau}^{\varphi \perp q}\right) \simeq\left(C_{0}(\mathbb{E} \perp V), J_{\sigma \perp \tau}^{a \cdot \varphi \perp a \cdot q}\right),
$$

where $(\mathbb{E}, a \cdot \varphi) \simeq[1, \delta]$. Note that $\left\{a^{-1} u, v\right\}$ is a standard symplectic basis of $(\mathbb{E}, a \cdot \varphi)$. Let $\left(\mathbb{E}^{\prime \prime}, \varphi^{\prime \prime}\right)$ be the quadratic plane $\left[1, \delta+\delta^{\prime}+\delta^{\prime}\right] \simeq[1, \delta] \simeq(\mathbb{E}, a \cdot \varphi)$ with a standard symplectic basis $\left\{x^{\prime}, y^{\prime}\right\}$. Set $t_{1}=\operatorname{dim} \operatorname{Fix}(\mathbb{E}, \sigma)-\frac{1}{2} \operatorname{dim} \mathbb{E}, t_{2}=$ $\operatorname{dim} \operatorname{Fix}(V, \tau)-\frac{1}{2} \operatorname{dim} V$ and $t_{1}^{\prime}=\operatorname{dim} \operatorname{Fix}\left(\mathbb{E}^{\prime}, \sigma^{\prime}\right)-\frac{1}{2} \operatorname{dim} \mathbb{E}^{\prime}$. We have $t=t_{1}+t_{2}$. By replacing $\mathbb{E}$ with $\mathbb{E}^{\prime}$ and $\sigma$ with $\sigma^{\prime}$ in $(i i)$, we obtain an isomorphism

$$
\left(C_{0}\left(\mathbb{E}^{\prime}\right), J_{\sigma^{\prime}}\right) \otimes\left(C(V), J_{\tau}^{a \cdot q}\right) \simeq\left(C_{0}\left(\mathbb{E}^{\prime \prime} \perp V\right), J_{\sigma^{\prime \prime}}^{\varphi^{\prime \prime} \perp \tau}\right),
$$

where $\sigma^{\prime \prime}$ is an involution in $O\left(\mathbb{E}^{\prime \prime}, \varphi^{\prime \prime}\right)$. Furthermore if $t_{1}^{\prime}+t_{2} \equiv 0 \bmod 2$ then $\sigma^{\prime \prime}=\tau_{x^{\prime}}$ and if $t_{1}^{\prime}+t_{2} \equiv 1 \bmod 2$ then $\sigma^{\prime \prime}=\operatorname{id}_{\mathbb{E}^{\prime \prime}}$.

Now the linear map $f: \mathbb{E} \rightarrow \mathbb{E}^{\prime \prime}$ defined by $f\left(a^{-1} u\right)=x^{\prime}$ and $f(v)=y^{\prime}$ induces an isomorphism $C(\mathbb{E}, a \cdot \varphi) \simeq C\left(\mathbb{E}^{\prime \prime}, \varphi^{\prime \prime}\right)$. Under this isomorphism, $\tau_{u}$ corresponds to $\tau_{x^{\prime}}$ and $\operatorname{id}_{\mathbb{E}}$ corresponds to id $\operatorname{E}_{\mathbb{E}^{\prime \prime}}$, i.e., $\sigma$ corresponds to $\sigma^{\prime \prime}$. So we can rewrite (2) as follows:

$$
\left(C_{0}\left(\mathbb{E}^{\prime}\right), J_{\sigma^{\prime}}\right) \otimes\left(C(V), J_{\tau}^{a \cdot q}\right) \simeq\left(C_{0}(\mathbb{E} \perp V), J_{\sigma \perp \tau}^{a \cdot \varphi \perp a \cdot q}\right),
$$

Furthermore if $t_{1}^{\prime}+t_{2} \equiv 0 \bmod 2$ then $\sigma=\tau_{u}$ (i.e., $t_{1}=0$ ) and if $t_{1}^{\prime}+t_{2} \equiv 1$ $\bmod 2$ then $\sigma=\operatorname{id} \mathbb{E}$ (i.e., $t_{1}=1$ ). So we have $t_{1}^{\prime}+t_{2} \equiv t_{1} \bmod 2$ which implies that $t=t_{1}+t_{2} \equiv t_{1}^{\prime} \bmod 2$.

Using (1) and (3) we obtain the desired isomorphism:

$$
\left(C_{0}(\mathbb{E} \perp V), J_{\sigma \perp \tau}^{\varphi \perp q}\right) \simeq\left(C_{0}\left(\mathbb{E}^{\prime}\right), J_{\sigma^{\prime}}\right) \otimes\left(C(V), J_{\tau}^{a \cdot q}\right) .
$$

Finally as $t \equiv t_{1}^{\prime} \bmod 2$, if $t \equiv 0 \bmod 2$ then $t_{1}^{\prime}=0$ (i.e., $\sigma^{\prime}=\tau_{x}$ ) and if $t \equiv 1$ $\bmod 2$ then $t_{1}^{\prime}=1$ (i.e., $\sigma^{\prime}=\operatorname{id}_{\mathbb{E}^{\prime}}$ ).

\section{Multiquaternion algebras with involution}

Let $(Q, \sigma)$ be a quaternion algebra with involution of the first kind. In [9] it has been shown that if char $F \neq 2$, then there exists a 2-dimensional quadratic space $(V, q)$ and an involution $\tau$ in $O(V, q)$ such that $(Q, \sigma) \simeq\left(C(V), J_{\tau}\right)$ (see $[9,(6.2)])$. Here we state this result for a field $F$ of characteristic 2 .

Theorem 6.1. Let $(Q, \sigma)$ be a quaternion algebra with an involution of the first kind over a field $F$ of characteristic 2 . Then there exists a quadratic plane 
$(\mathbb{E}, \varphi)$ over $F$ with an involution $\tau \in O(\mathbb{E}, \varphi)$ such that $(Q, \sigma) \simeq\left(C(\mathbb{E}), J_{\tau}\right)$. Furthermore if $\sigma$ is of symplectic type, then $\tau$ is necessarily equal to id and if $\sigma$ is of orthogonal type, then $\tau \neq$ id is necessarily a reflection.

Proof. Let $Q=[\alpha, \beta)$ for $\alpha \in F, \beta \in F^{\times}$and consider a standard basis $\{1, i, j, k\}$ of $Q$. Let $\gamma$ be the canonical involution of $Q$. Then by [14, Ch. 8, (7.4)], there exists some $x \in Q^{*}$ with $\gamma(x)=x$ such that $\sigma=\operatorname{Int}(x) \circ \gamma$. So if we write $x=a+b i+c j+d k$ for some $a, b, c, d \in F$, then $b=0$ and we obtain $x=a+c j+d k$. If $c=d=0$, then $\sigma=\gamma$. Let $\mathbb{E}$ be the quadratic plane $\left[\beta, \alpha \beta^{-1}\right]$ over $F$. By $[14$, Ch. 9, (4.6)], we have $Q \simeq C(\mathbb{E})$. Using (4.3), the involution $J_{\text {id }}$ on $C(\mathbb{E})$ is of symplectic type. Since $\gamma$ is the unique involution of symplectic type on $Q$, we have $(Q, \gamma) \simeq\left(C(\mathbb{E}), J_{\text {id }}\right)$.

Now suppose that $c$ or $d$ is nonzero, then there exists $s, t \in F$ such that $c s+d t=a$. Let $(\mathbb{E}, \varphi)$ be a quadratic plane over $F$ with a basis $\{u, v\}$ and the relations $\varphi(u)=t^{2}+\alpha \beta, \varphi(v)=s^{2}+\beta$ and $B(u, v)=\beta \neq 0$. Define the linear map $f: \mathbb{E} \rightarrow Q$ by $f(u)=t+k$ and $f(v)=s+j$. We have $f(u)^{2}=(t+k)^{2}=t^{2}+\alpha \beta=\varphi(u)$ and $f(v)^{2}=(s+j)^{2}=s^{2}+\beta=\varphi(v)$. We also have

$$
\begin{aligned}
f(u) f(v)+f(v) f(u) & =(t+k)(s+j)+(s+j)(t+k)=k j+j k \\
& =i j j+j i j=(i j+j i) j=j^{2}=\beta=B(u, v) .
\end{aligned}
$$

So the map $f$ is compatible with $\varphi$ and it can be extended to an isomorphism $\psi: C(\mathbb{E}) \simeq Q$. Let $I=\psi^{-1} \circ \gamma \circ \psi$. Then $I$ is an involution on $C(\mathbb{E})$ and $(Q, \gamma) \simeq(C(\mathbb{E}), I)$. We have the following relations:

$$
\begin{aligned}
& I(u)=\psi^{-1} \circ \gamma \circ \psi(u)=\psi^{-1} \circ \gamma(t+k)=\psi^{-1}(t+k)=u, \\
& I(v)=\psi^{-1} \circ \gamma \circ \psi(v)=\psi^{-1} \circ \gamma(s+j)=\psi^{-1}(s+j)=v .
\end{aligned}
$$

So $I=J_{\operatorname{id}_{\mathbb{E}}}$. Now let $T=\psi^{-1} \circ \sigma \circ \psi$. Then $T$ is an involution on $C(\mathbb{E})$ and $(Q, \sigma) \simeq(C(\mathbb{E}), T)$. We have $\psi(d u+c v)=d(t+k)+c(s+j)=a+c j+d k=x$, so $\psi^{-1}(x)=d u+c v \in \mathbb{E}$. Since $\sigma=\operatorname{Int}(x) \circ \gamma$, we obtain $T=\psi^{-1} \circ \sigma \circ \psi=$ $\psi^{-1} \circ \operatorname{Int}(x) \circ \gamma \circ \psi=\left(\psi^{-1} \circ \operatorname{Int}(x) \circ \psi\right) \circ\left(\psi^{-1} \circ \gamma \circ \psi\right)=\operatorname{Int}\left(\psi^{-1}(x)\right) \circ J_{\mathrm{id}_{\mathbb{E}}}$. By (4.1), in order to show that $T=J_{\tau}$ for some involution in $O(\mathbb{E}, \varphi)$, it is enough to show that $T(\mathbb{E})=\mathbb{E}$ or equivalently $T(w)=\operatorname{Int}\left(\psi^{-1}(x)\right) \circ J_{\mathrm{id}_{\mathbb{E}}}(w)=$ $\operatorname{Int}\left(\psi^{-1}(x)\right)(w) \in \mathbb{E}$ for every $w \in \mathbb{E}$. But since $x \in Q^{*}$, we get $x^{2} \neq 0$, so $\varphi\left(\psi^{-1}(x)\right)=\psi^{-1}(x)^{2} \neq 0$, i.e., $\psi^{-1}(x)$ is anisotropic. On the other hand we have $\psi^{-1}(x) \in \mathbb{E}$, so $\left.\operatorname{Int}\left(\psi^{-1}(x)\right)\right|_{\mathbb{E}}$ is a reflection and $T(\mathbb{E})=\mathbb{E}$. So $\sigma=J_{\tau}$ for some isometry $\tau$ of $(\mathbb{E}, \varphi)$.

This proves the first part of the result. The second part follows from (4.3).

Corollary 6.2. Let $(\mathbb{E}, \varphi, B)$ and $\left(\mathbb{E}^{\prime}, \varphi^{\prime}, B^{\prime}\right)$ be two quadratic planes over a field $F$ of characteristic 2 . If $\tau$ and $\tau^{\prime}$ are reflections of $(\mathbb{E}, \varphi)$ and $\left(\mathbb{E}^{\prime}, \varphi^{\prime}\right)$, respectively, then the following statements are equivalent: $(1)\left(C(\mathbb{E}), J_{\tau}\right) \simeq$ $\left(C\left(\mathbb{E}^{\prime}\right), J_{\tau^{\prime}}\right)$. (2) $C(\mathbb{E}) \simeq C\left(\mathbb{E}^{\prime}\right)$ and $\theta(\tau)=\theta\left(\tau^{\prime}\right)$.

Proof. According to (4.11), we have $\operatorname{disc} J_{\tau}=\theta(\tau)$ and $\operatorname{disc} J_{\tau^{\prime}}=\theta\left(\tau^{\prime}\right)$. The implication $(1) \Rightarrow(2)$ is immediate and $(2) \Rightarrow(1)$ follows from $[6,(7.4)]$.

The next result is analogous to $[9,(6.3)]$. 
Theorem 6.3. Let $F$ be a field of characteristic 2 and let $\left(Q_{1}, \sigma_{1}\right), \cdots,\left(Q_{n}, \sigma_{n}\right)$ be quaternion algebras over $F$ with involutions of the first kind. Let $(A, \sigma) \simeq$ $\left(Q_{1}, \sigma_{1}\right) \otimes \cdots \otimes\left(Q_{n}, \sigma_{n}\right)$. Then there is a quadratic space $(V, q)$ of dimension $2 n$ over $F$ and an involution $\tau$ in $O(V, q)$ such that $(A, \sigma) \simeq\left(C(V), J_{\tau}\right)$. Furthermore if $\sigma$ is of orthogonal type then $\operatorname{dim} \operatorname{Fix}(V, \tau)=\frac{1}{2} \operatorname{dim} V$ and $\tau$ can be chosen to be an orthogonal sum of reflections and if $\sigma$ is of symplectic type then $\tau$ can be chosen to be identity.

Proof. By (6.1), for each $1 \leqslant i \leqslant n$ there is a quadratic plane $\left(\mathbb{E}_{i}, \varphi_{i}\right)$ with an involution $\tau_{i} \in O\left(\mathbb{E}_{i}, \varphi_{i}\right)$ such that $\left(Q_{i}, \sigma_{i}\right) \simeq\left(C\left(\mathbb{E}_{i}\right), J_{\tau_{i}}\right)$ and by (4.4), $(A, \sigma) \simeq\left(C(V), J_{\tau}\right)$ where $(V, q)=\left(\mathbb{E}_{1}, \varphi_{1}\right) \perp \cdots \perp\left(\mathbb{E}_{n}, \varphi_{n}\right)$ and $\tau=\tau_{1} \perp$ $\cdots \perp \tau_{n}$. If $\sigma$ is of orthogonal type then by [6, (2.23)], each $\sigma_{i}$ is of orthogonal type and by (6.1), $\tau_{i}$ is a reflection for each $i$, so $\tau$ is an orthogonal sum of $n$ reflections and $\operatorname{dim} \operatorname{Fix}(V, \tau)=\frac{1}{2} \operatorname{dim} V$. If $\sigma$ is of symplectic type then by $[6,(2.23)]$, at least one of the $\sigma_{i}$ 's is of symplectic type, say $\sigma_{1}$. Then by (6.1), $\left(Q_{1}, \sigma_{1}\right) \simeq\left(C\left(\mathbb{E}_{1}\right), J_{\text {id }}\right)$. Now if $\sigma_{2}$ is of symplectic type then $\left(Q_{1}, \sigma_{1}\right) \otimes$ $\left(Q_{2}, \sigma_{2}\right) \simeq\left(C\left(\mathbb{E}_{1}\right), J_{\mathrm{id}}\right) \otimes\left(C\left(\mathbb{E}_{2}\right), J_{\mathrm{id}}\right) \simeq\left(C\left(\mathbb{E}_{1} \perp \mathbb{E}_{2}\right), J_{\mathrm{id}}\right)$. If $\sigma_{2}$ is of orthogonal type, one can write $\left(Q_{2}, \sigma_{2}\right) \simeq\left(C\left(\mathbb{E}_{2}\right), J_{\tau_{2}}\right)$ where $\tau_{2}$ is a reflection of $\mathbb{E}_{2}$. So $\left(Q_{1}, \sigma_{1}\right) \otimes\left(Q_{2}, \sigma_{2}\right) \simeq\left(C\left(\mathbb{E}_{1}\right), J_{\mathrm{id}}\right) \otimes\left(C\left(\mathbb{E}_{2}\right), J_{\tau_{2}}\right) \simeq\left(C\left(\mathbb{E}_{1} \perp \mathbb{E}_{2}\right), J_{\mathrm{id} \perp \tau_{2}}\right)$. By (5.2) this is isomorphic to $\left(C(V), J_{\text {id }}\right)$ for a suitable 4-dimensional quadratic space $(V, q)$ over $F$. Now induction on $n$ completes the proof.

Lemma 6.4. Let $K / F$ be a separable quadratic extension of fields of characteristic 2 with the nontrivial automorphism $\rho$. Then there is a quadratic plane $(\mathbb{E}, \varphi)$ over $F$ such that $(K, \rho) \simeq\left(C_{0}(\mathbb{E}), J_{\mathrm{id}}\right)$. Furthermore if $(\mathbb{E}, \varphi)$ is a quadratic plane over $F$ and $\tau$ is an involution in $O(\mathbb{E}, \varphi)$ such that $(K, \rho) \simeq\left(C_{0}(\mathbb{E}), J_{\tau}\right)$, then $\tau$ is necessarily identity.

Proof. Since $K / F$ is separable we may assume $K=F(x)$ where $x^{2}+x=\delta \in F^{\times}$. Let $(\mathbb{E}, \varphi)$ be the quadratic space $[1, \delta]$ with a standard symplectic basis $\{u, v\}$. Define $f: K \rightarrow C_{0}(\mathbb{E})$ via $f(\lambda+\mu x)=\lambda+\mu u v$ for every $\lambda, \mu \in F$. We have $f(x)^{2}=(u v)^{2}=u v+\delta=f(x+\delta)=f\left(x^{2}\right)$. So for every $\lambda_{1}, \mu_{1}, \lambda_{2}, \mu_{2} \in F$ we have $f\left(\left(\lambda_{1}+\mu_{1} x\right)\left(\lambda_{2}+\mu_{2} x\right)\right)=f\left(\left(\lambda_{1}+\mu_{1} u v\right)\left(\lambda_{2}+\mu_{2} u v\right)\right)$. Hence $f$ is an $F$-algebra homomorphism and in fact an isomorphism, because it is obviously surjective. We have

$$
J_{\mathrm{id}_{\mathbb{E}}} \circ f(x)=J_{\mathrm{id}_{\mathbb{E}}}(u v)=v u=u v+1=f(x+1)=f \circ \rho(x) .
$$

So $(K, \rho) \simeq\left(C_{0}(\mathbb{E}), J_{\mathrm{id}}\right)$.

Now suppose that $(K, \rho) \simeq\left(C_{0}(\mathbb{E}), J_{\tau}\right)$ for a quadratic plane $(\mathbb{E}, \varphi)$ and an involution $\tau \in O(\mathbb{E}, \varphi)$. As $\rho(x)=x+1$, there is $y \in C_{0}(\mathbb{E}) \subseteq C(\mathbb{E})$ such that $J_{\tau}(y)=y+1$, i.e., $1 \in \operatorname{Alt}\left(C(\mathbb{E}), J_{\tau}\right)$, so by $[6,(2.6)],\left(C(\mathbb{E}), J_{\tau}\right)$ is of symplectic type and hence by $(4.3), \tau=\mathrm{id}$.

Remark 6.5. In contrast with the case where char $F \neq 2$, if $(V, q)$ is a $2 n$ dimensional quadratic space over a field $F$ of characteristic 2 , then there is no quadratic form $q^{\prime}: V^{\prime} \rightarrow F$ with $\operatorname{dim} V^{\prime}=2 n-1$ such that $C_{0}(V) \cong C\left(V^{\prime}\right)$. In fact suppose that there is an $F$-algebra isomorphism $f: C\left(V^{\prime}\right) \simeq C_{0}(V)$. Since $\operatorname{dim} V^{\prime}$ is odd, $\left(V^{\prime}, q^{\prime}\right)$ is not regular and there is an element $u \in V^{\prime}$ such that $B(u, v)=0$ for all $v \in V^{\prime}$ and hence $u \in Z\left(C\left(V^{\prime}\right)\right)$. So we must have $f(u) \in Z\left(C_{0}(V)\right)$. Therefore, we can write $f(u)=\lambda+\mu\left(u_{1} v_{1}+\cdots+\right.$ $\left.u_{n} v_{n}\right)$ where $\left\{u_{1}, v_{1}, \cdots, u_{n}, v_{n}\right\}$ is a symplectic basis of $(V, q)$. Since $f$ is an 
$F$-isomorphism, $\mu \neq 0$. On the other hand we have $u^{2}=q^{\prime}(u) \in F$, but $f(u)^{2}=\lambda^{2}+\mu^{2}\left(q\left(u_{1}\right) q\left(v_{1}\right)+\cdots+q\left(u_{n}\right) q\left(v_{n}\right)+u_{1} v_{1}+\cdots+u_{n} v_{n}\right) \notin F$ which is a contradiction.

Remark 6.6. Let $K / F$ be a separable quadratic extension of fields with the nontrivial automorphism $\rho$. If $\operatorname{char} F \neq 2$, then there is a 1-dimensional quadratic space over $F$ such that $K \simeq C(V)$. But if $\operatorname{char} F=2$, then (6.4) and (6.5) show that there is no 1-dimensional quadratic space $(V, q)$ over $F$ such that $K \simeq C(V)$.

Theorem 6.7. Let $F$ be a field of characteristic 2 and let $\left(Q_{1}, \sigma_{1}\right), \cdots,\left(Q_{n}, \sigma_{n}\right)$ be quaternion algebras over $F$ with involutions of the first kind. Let $K / F$ be a separable quadratic extension with the nontrivial automorphism $\rho$. If $(A, \sigma) \simeq$ $\left(Q_{1}, \sigma_{1}\right) \otimes \cdots \otimes\left(Q_{n}, \sigma_{n}\right) \otimes(K, \rho)$, then there is a quadratic space $(V, q)$ of dimension $2 n+2$ over $F$ and an involution $\tau$ in $O(V, q)$ such that $(A, \sigma) \simeq\left(C_{0}(V), J_{\tau}\right)$. Furthermore if $n \equiv 0 \bmod 2$, then $\tau$ can be chosen to be identity and if $n \equiv 1$ $\bmod 2$, then $\tau$ can be chosen to be a reflection.

Proof. By (6.3), there is a quadratic space $\left(V^{\prime}, q^{\prime}\right)$ of dimension $2 n$ over $F$ and an involution $\tau^{\prime} \in O\left(V^{\prime}, q^{\prime}\right)$ such that $\left(Q_{1}, \sigma_{1}\right) \otimes \cdots \otimes\left(Q_{n}, \sigma_{n}\right) \simeq\left(C\left(V^{\prime}\right), J_{\tau^{\prime}}\right)$. Also by $(6.4)$, there is a quadratic plane $(\mathbb{E}, \varphi)$ over $F$ such that $(K, \rho) \simeq\left(C_{0}(\mathbb{E}), J_{\text {id }}\right)$. By (6.3), we can choose $\left(V^{\prime}, \tau^{\prime}\right)$ such that either $\tau^{\prime}=\mathrm{id}_{V^{\prime}}$ or $\tau^{\prime}=\tau_{1}^{\prime} \perp \cdots \perp \tau_{n}^{\prime}$ where $\tau_{i}^{\prime}, i=1, \cdots, n$, is a reflection of some quadratic subplane $\mathbb{E}_{i}^{\prime}$ of $V^{\prime}$. Set $t=\operatorname{dim} \operatorname{Fix}\left(V^{\prime} \perp \mathbb{E}, \tau^{\prime} \perp\right.$ id $)-\frac{1}{2} \operatorname{dim}\left(V^{\prime} \perp \mathbb{E}\right)$.

First suppose that $\tau^{\prime}=\mathrm{id}_{V^{\prime}}$. We have $t=n+1$. If $n \equiv 0 \bmod 2$, then by $(5.3(i i))$, there exists a quadratic plane $\left(\mathbb{E}^{\prime}, \varphi^{\prime}\right)$ over $F$ such that $(A, \sigma) \simeq$ $\left(C\left(V^{\prime}\right), J_{\text {id }}\right) \otimes\left(C_{0}(\mathbb{E}), J_{\text {id }}\right) \simeq\left(C_{0}\left(V^{\prime} \perp \mathbb{E}^{\prime}\right), J_{\text {id } \perp \text { id }}\right)$. So $(A, \sigma) \simeq\left(C_{0}(V), J_{\text {id }}\right)$ for $V=V^{\prime} \perp \mathbb{E}^{\prime}$. If $n \equiv 1 \bmod 2$, then by (5.3 (ii)), there exists a quadratic space $\left(\mathbb{E}^{\prime}, \varphi^{\prime}\right)$ over $F$ with a reflection $\tau^{\prime \prime}$ such that $(A, \sigma) \simeq\left(C\left(V^{\prime}\right), J_{\text {id }}\right) \otimes$ $\left(C_{0}(\mathbb{E}), J_{\mathrm{id}}\right) \simeq\left(C_{0}\left(V^{\prime} \perp \mathbb{E}^{\prime}\right), J_{\mathrm{id} \perp \tau^{\prime \prime}}\right)$. So $(A, \sigma) \simeq\left(C_{0}(V), J_{\tau}\right)$ for $V=V^{\prime} \perp \mathbb{E}^{\prime}$ and the reflection $\tau=\mathrm{id}_{V^{\prime}} \perp \tau^{\prime \prime}$.

Now suppose that $\tau^{\prime}=\tau_{1}^{\prime} \perp \cdots \perp \tau_{n}^{\prime}$. We have $t=1$, so by $(5.3(i i))$, there exists a quadratic space $\left(\mathbb{E}^{\prime}, \varphi^{\prime}\right)$ over $F$ such that $(A, \sigma) \simeq\left(C\left(V^{\prime}\right), J_{\tau^{\prime}}\right) \otimes$ $\left(C_{0}(\mathbb{E}), J_{\text {id }}\right) \simeq\left(C_{0}\left(V^{\prime} \perp \mathbb{E}^{\prime}\right), J_{\tau^{\prime} \perp \text { id }_{\mathbb{E}^{\prime}}}\right)$. If $\operatorname{dim} V^{\prime}=2$, then $(A, \sigma) \simeq\left(C_{0}(V), J_{\tau}\right)$ where $V=V^{\prime} \perp \mathbb{E}^{\prime}$ and $\tau=\tau^{\prime} \perp \mathrm{id}_{\mathbb{E}^{\prime}}$ is a reflection of $V$. So suppose that $\operatorname{dim} V^{\prime} \geqslant 4$. We have

$$
(A, \sigma) \simeq\left(C_{0}\left(V^{\prime} \perp \mathbb{E}^{\prime}\right), J_{\tau^{\prime} \perp \mathrm{id}_{\mathbb{E}^{\prime}}}\right) \simeq\left(C_{0}\left(\mathbb{E}_{1}^{\prime} \perp W\right), J_{\tau_{1}^{\prime} \perp \rho}\right),
$$

where $W=\mathbb{E}_{2}^{\prime} \perp \cdots \perp \mathbb{E}_{n}^{\prime} \perp \mathbb{E}^{\prime}$ and $\rho=\tau_{2}^{\prime} \perp \cdots \perp \tau_{n}^{\prime} \perp \operatorname{id}_{\mathbb{E}^{\prime}}$. We have $\operatorname{dim} \operatorname{Fix}\left(\mathbb{E}_{1}^{\prime} \perp W, \tau_{1}^{\prime} \perp \rho\right)-\frac{1}{2} \operatorname{dim}\left(\mathbb{E}_{1}^{\prime} \perp W\right)=1$, so by $(5.3$ (iii)), $\left(C_{0}\left(\mathbb{E}_{1}^{\prime} \perp W\right), J_{\tau_{1}^{\prime} \perp \rho}\right) \simeq\left(C_{0}\left(\mathbb{E}^{\prime \prime}\right), J_{\mathrm{id}}\right) \otimes\left(C\left(W, a \cdot q_{1}\right), J_{\rho}\right)$ where $\left(\mathbb{E}^{\prime \prime}, \varphi^{\prime \prime}\right)$ is a suitable quadratic space over $F, q_{1}$ is the restriction of $q^{\prime} \perp \varphi^{\prime}$ to $W$ and $a \in D_{F}\left(\left.q\right|_{\mathbb{E}_{1}^{\prime}}\right)$. Since $\operatorname{dim} \operatorname{Fix}(W, \rho)=n+1>n=\frac{1}{2} \operatorname{dim} W$, by (4.7), the involution $J_{\rho}$ on $C(W)$ is of symplectic type. So by (6.3), there exists a quadratic space $\left(V^{\prime \prime}, q^{\prime \prime}\right)$ over $F$ such that $\left(C\left(W, a \cdot q_{1}\right), J_{\rho}\right) \simeq\left(C\left(V^{\prime \prime}\right), J_{\text {id }}\right)$. So we have $(A, \sigma) \simeq\left(C_{0}\left(\mathbb{E}^{\prime \prime}\right), J_{\text {id }}\right) \otimes\left(C\left(V^{\prime \prime}\right), J_{\text {id }}\right)$. Now by an argument similar to the case $\tau^{\prime}=\operatorname{id}_{V^{\prime}}$, we have $(A, \sigma) \simeq\left(C_{0}(V), J_{\text {id }}\right)$ if $n \equiv 0 \bmod 2$, and $(A, \sigma) \simeq\left(C_{0}(V), J_{\tau}\right)$ for some reflection $\tau$ if $n \equiv 1 \bmod 2$ and the result is proved. 
Corollary 6.8. Let $(Q, \sigma)$ be a quaternion algebra with involution of the second kind over a field $K$ of characteristic 2. Let $F \subseteq K$ be the fixed subfield of $\left.\sigma\right|_{K}$. Then there exists a 4-dimensional quadratic space $(\mathbb{A}, q)$ over $F$ such that $(Q, \sigma) \simeq\left(C_{0}(\mathbb{A}), J_{\tau}\right)$ where $\tau$ is a suitable reflection of $(\mathbb{A}, q)$. ([5]).

Proof. The result follows from [14, Ch. 8, (11.2)] and (6.7).

Remark 6.9. With the notation of (6.7), if $n \equiv 1 \bmod 2$ then the involution $\tau \in O(V, q)$ can not be chosen to be identity; because in this case $\operatorname{dim} V=$ $2 n+2 \equiv 0 \bmod 4$ and $J_{\text {id }}$ is an involution on $C(V)$ of the first kind, i.e., the involution $\sigma$ is of the first kind which contradicts the assumption. In particular the involution $\tau$ of (6.8) can not be chosen to be identity. Also there is no $(2 n-1)$-dimensional quadratic space $(V, q)$ over $F$ such that $A \simeq C(V)$. This fact is an easy consequence of (6.5) and (6.7).

Proposition 6.10. Let $(\mathbb{A}, q)$ be a 4-dimensional quadratic space over a field $F$ of characteristic 2 and let $\tau$ be an interchange isometry of $(\mathbb{A}, q)$. Then $\left(C(\mathbb{A}), J_{\tau}\right) \simeq\left(C\left(\mathbb{H}_{1}\right), J_{\tau_{u_{1}}}\right) \otimes\left(C\left(\mathbb{H}_{2}\right), J_{\tau_{u_{2}}}\right)$ where $\left(\mathbb{H}_{1}, \varphi_{1}\right)$ and $\left(\mathbb{H}_{2}, \varphi_{2}\right)$ are isometric to the hyperbolic plane $[1,0]$ over $F$ with respective standard symplectic bases $\left\{u_{1}, v_{1}\right\}$ and $\left\{u_{2}, v_{2}\right\}$ and $\tau_{u_{i}}, i=1,2$, is the reflection along the anisotropic vector $u_{i} \in \mathbb{H}_{i}$. In particular $\theta\left(\tau_{u_{1}}\right)=\theta\left(\tau_{u_{2}}\right)=1 \in F^{\times} / F^{\times 2}$. Furthermore if $\{w, x, y, z\}$ is a basis of $\mathbb{A}$ with the properties stated in (3.5), then $\left(C\left(\mathbb{H}_{1}\right), J_{\tau_{u_{1}}}\right)$ (resp. $\left.\left(C\left(\mathbb{H}_{2}\right), J_{\tau_{u_{2}}}\right)\right)$ is isomorphic, as an F-algebra with involution, to the $F$-quaternion algebra generated by $\{1+x+w+x w, y+x z+x z w\}$ (resp. $\{1+x+x w, y+x z+x z w+z+z w\})$, which are invariant under $J_{\tau}$.

Proof. Let $\{w, x, y, z\}$ be a basis of $\mathbb{A}$ with the properties stated in (3.5), i.e., $q(w)=q(x)=q(y)=q(z)=0, \tau(w)=w, \tau(x)=x, \tau(y)=x+y, \tau(z)=$ $w+z, B(w, y)=B(x, z)=1$ and all other pairs of vectors from this basis are orthogonal. Set $\left(\mathbb{A}^{\prime}, q^{\prime}, B^{\prime}\right)=\left(\mathbb{H}_{1}, \varphi_{1}\right) \perp\left(\mathbb{H}_{2}, \varphi_{2}\right)$. Then the set $\mathcal{B}=$ $\left\{u_{1}, v_{1}, u_{2}, v_{2}\right\}$ is a symplectic basis of $\mathbb{A}^{\prime}$. Define the linear map $f: \mathbb{A}^{\prime} \rightarrow C(\mathbb{A})$ by $f\left(u_{1}\right)=1+x+w+x w, f\left(v_{1}\right)=y+x z+x z w, f\left(u_{2}\right)=1+x+x w$ and $f\left(v_{2}\right)=y+x z+x z w+z+z w=f\left(v_{1}\right)+z+z w$. Using the above relations and straightforward calculations in Clifford algebra we obtain that for every $x, y \in \mathcal{B}$, we have $f(x)^{2}=q^{\prime}(x)$ and $f(x) f(y)+f(y) f(x)=B^{\prime}(x, y)$. So the map $f$ is compatible with $q^{\prime}$ and it can be extended to an isomorphism $\psi: C\left(\mathbb{A}^{\prime}\right) \simeq C(\mathbb{A})$. We claim that for every $v \in C\left(\mathbb{A}^{\prime}\right), J_{\tau} \circ \psi(v)=\psi \circ\left(J_{\tau_{u_{1}}} \perp J_{\tau_{u_{2}}}\right)(v)$. It is enough to check this for $v=u_{1}, v=v_{1}, v=u_{2}$ and $v=v_{2}$. We have the following relations:

$$
\begin{gathered}
J_{\tau} \circ \psi\left(u_{1}\right)=J_{\tau}(1+x+w+x w)=1+x+w+w x \\
=\psi\left(u_{1}\right)=\psi \circ\left(J_{\tau_{u_{1}}} \perp J_{\tau_{u_{2}}}\right)\left(u_{1}\right) \\
J_{\tau} \circ \psi\left(u_{2}\right)=J_{\tau}(1+x+x w)=1+x+w x=\psi\left(u_{2}\right)=\psi \circ\left(J_{\tau_{u_{1}}} \perp J_{\tau_{u_{2}}}\right)\left(u_{2}\right), \\
J_{\tau} \circ \psi\left(v_{1}\right)=J_{\tau}(y+x z+x z w)=(x+y)+(w+z) x+w(w+z) x \\
=(x+y)+(w x+z x)+(q(w) x+w z x) \\
=(x+y)+(x w+x z+1)+(0+x z w+w) \\
=(y+x z+x z w)+(1+x+w+x w)=\psi\left(v_{1}+u_{1}\right) \\
=\psi\left(v_{1}+\frac{1}{q\left(u_{1}\right)} u_{1}\right)=\psi \circ\left(J_{\tau_{u_{1}}} \perp J_{\tau_{u_{2}}}\right)\left(v_{1}\right)
\end{gathered}
$$




$$
\begin{aligned}
J_{\tau} \circ \psi\left(v_{2}\right) & =J_{\tau}(y+x z+x z w+z+z w) \\
& =(x+y)+(w+z) x+w(w+z) x+(w+z)+w(w+z) \\
& =(x+y)+(w x+z x)+(q(w) x+w z x)+(w+z)+(q(w)+w z) \\
& =(x+y)+(x w+x z+1)+(0+x z w+w)+(w+z)+(0+z w) \\
& =(y+x z+x z w+z+z w)+(1+x+x w)=\psi\left(v_{2}+u_{2}\right) \\
& =\psi\left(v_{2}+\frac{1}{q\left(u_{2}\right)} u_{2}\right)=\psi \circ\left(J_{\tau_{u_{1}}} \perp J_{\tau_{u_{2}}}\right)\left(v_{2}\right) .
\end{aligned}
$$

Finally note that $\theta\left(\tau_{u_{1}}\right)=q\left(u_{1}\right) F^{\times 2}=1 \in F^{\times} / F^{\times 2}$ and $\theta\left(\tau_{u_{2}}\right)=q\left(u_{2}\right) F^{\times 2}=$ $1 \in F^{\times} / F^{\times 2}$ and the result is proved.

Remark 6.11. Using (6.10), one can give a shorter proof for (4.6) and (4.11 (b)).

\section{Involutions of an even Clifford algebra with trivial Arf invariant}

Remark 7.1. Let $F$ be a field and let $(A, \sigma)$ and $(B, \tau)$ be two $F$-algebras with involutions. Let $A \times B$ be the direct product of $A$ and $B$. The involution on $A \times B$ defined by $(a, b) \mapsto(\sigma(a), \tau(b))$ is denoted by $\sigma \times \tau$.

The proof of the next result follows from the standard properties of tensor and direct product and we left it to the reader.

Lemma 7.2. Let $F$ be a field and let $(A, \sigma),(B, \tau)$ and $(C, \rho)$ be $F$-algebras with involution. Then there exists an isomorphism of $F$-algebras with involution $\left((A \times B) \otimes_{F} C,(\sigma \times \tau) \otimes \rho\right) \simeq\left(\left(A \otimes_{F} C\right) \times\left(B \otimes_{F} C\right),(\sigma \otimes \rho) \times(\tau \otimes \rho)\right)$.

Remark 7.3. Let $(V, q)$ be a quadratic space with trivial Arf invariant and let $\tau$ be an involution in $O(V, q)$. We claim that there is a $\tau$-symplectic basis $\left\{u_{1}, v_{1}, \cdots, u_{n}, v_{n}\right\}$ of $V$ such that $\sum_{i=1}^{n} q\left(u_{i}\right) q\left(v_{i}\right)=0$. If $\tau$ is an orthogonal sum of interchange isometries, then by (3.6), $V=\mathbb{H}_{1} \perp \cdots \cdots \mathbb{H}_{2 s}$. Choose a standard symplectic basis $\left\{u_{i}, v_{i}\right\}$ of $\mathbb{H}_{i} \simeq[1,0], i=1, \cdots, 2 s$. Then $\left\{u_{1}, v_{1}, \cdots, u_{n}, v_{n}\right\}$ is the desired basis. Otherwise there exists either an identity map or a reflection in the Wiitala decomposition of $\tau$. So there is a $\tau$-symplectic basis $\left\{u_{1}, v_{1}, \cdots, u_{n}, v_{n}\right\}$ of $V$ such that $q\left(u_{1}\right) \neq 0$. We have $\sum_{i=1}^{n} q\left(u_{i}\right) q\left(v_{i}\right)=a^{2}+a \in \wp(F)$, where $a \in F$. If $a \neq 0$, by replacing $v_{1}$ with $v_{1}^{\prime}=v_{1}+a q\left(u_{1}\right)^{-1} u_{1}$, we have $\sum_{i=1}^{n} q\left(u_{i}\right) q\left(v_{i}\right)=0$.

Lemma 7.4. For $n \geqslant 2$, let $\left(\mathbb{A}_{1}, q_{1}\right), \cdots,\left(\mathbb{A}_{n}, q_{n}\right)$ be 4-dimensional hyperbolic spaces over a field $F$ of characteristic 2 . Then we have $C_{0}\left(\mathbb{A}_{1} \perp \cdots \perp \mathbb{A}_{n}\right) \simeq$ $C_{0}\left(\mathbb{A}_{1}\right) \otimes C\left(\mathbb{A}_{2} \perp \cdots \perp \mathbb{A}_{n}\right)$.

Proof. For every $1 \leqslant i \leqslant n$, there exist hyperbolic planes $\mathbb{H}_{2 i-1}$ and $\mathbb{H}_{2 i}$ such that $\mathbb{A}_{i}=\mathbb{H}_{2 i-1} \perp \mathbb{H}_{2 i}$. Let $\left\{u_{i}, v_{i}\right\}, i=1, \cdots, 2 n$, be a standard symplectic basis of $\mathbb{H}_{i} \simeq[1,0]$. Set $\left(W_{1}, q_{1}\right)=\mathbb{H}_{1} \perp \mathbb{H}_{3} \perp \cdots \perp \mathbb{H}_{2 n-1}$ and $\left(W_{2}, q_{2}\right)=$ $\mathbb{H}_{2} \perp \mathbb{H}_{4} \perp \cdots \perp \mathbb{H}_{2 n}$.

Define the linear map $f_{1}: W_{1} \rightarrow C\left(W_{1}\right)$ via $f_{1}\left(u_{1}\right)=u_{1}, f_{1}\left(v_{1}\right)=v_{1}+$ $u_{1}\left(u_{3} v_{3}+\cdots+u_{2 n-1} v_{2 n-1}\right), f_{1}\left(u_{2 i-1}\right)=u_{1} u_{2 i-1}$ and $f_{1}\left(v_{2 i-1}\right)=u_{1} v_{2 i-1}$ for $i=2, \cdots, n$. 
Similarly define the linear map $f_{2}: W_{2} \rightarrow C\left(W_{2}\right)$ via $f_{2}\left(u_{2}\right)=u_{2}, f_{2}\left(v_{2}\right)=$ $v_{2}+u_{2}\left(u_{4} v_{4}+\cdots+u_{2 n} v_{2 n}\right), f_{2}\left(u_{2 i}\right)=u_{2} u_{2 i}$ and $f_{2}\left(v_{2 i}\right)=u_{2} v_{2 i}$ for $i=2, \cdots, n$.

By the proof of (5.1), the maps $f_{1}$ and $f_{2}$ are compatible with $q_{1}$ and $q_{2}$, respectively; for example for the map $f_{1}$, considering the notation of the proof of (5.1) we have $\mathbb{E}^{\prime}=\mathbb{E}=\mathbb{H}_{1}, V=\mathbb{H}_{3} \perp \cdots \perp \mathbb{H}_{2 n-1}, u=x=u_{1}, v=y=v_{1}$, $a=q\left(u_{1}\right)=1, x^{-1}=u_{1}$ and $\delta=q\left(u_{3}\right) q\left(v_{3}\right)+\cdots+q\left(u_{2 n-1}\right) q\left(v_{2 n-1}\right)=0$. Now define the linear map $f_{1}^{\prime}: W_{1} \rightarrow C\left(W_{1}\right)$ the same as $f_{1}$ except that $f_{1}^{\prime}\left(v_{1}\right)=v_{1}+u_{1}\left(u_{3} v_{3}+\cdots+u_{2 n-1} v_{2 n-1}+(n-1) \cdot 1\right)=f_{1}\left(v_{1}\right)+(n-1) \cdot u_{1}$. We claim that $f_{1}^{\prime}$ is also compatible with $q_{1}$. It is enough to show that for every $x, y \in\left\{u_{1}, v_{1}, \cdots, u_{2 n-1}, v_{2 n-1}\right\}$, we have $f_{1}^{\prime}(x)^{2}=q_{1}(x)$ and $f_{1}^{\prime}(x) f_{1}^{\prime}(y)+$ $f_{1}^{\prime}(y) f_{1}^{\prime}(x)=B_{q_{1}}(x, y)$. It is clear that $(n-1) \cdot u_{1}=f_{1}^{\prime}\left(v_{1}\right)-f_{1}\left(v_{1}\right)$ commutes with $f_{1}\left(u_{1}\right), f_{1}\left(u_{2 i-1}\right)$ and $f_{1}\left(v_{2 i-1}\right)$ for $i=2, \cdots, n$. Since $f_{1}$ is compatible with $q_{1}$, in order to see that $f_{1}^{\prime}$ is compatible with $q_{1}$, it is enough to show that $f_{1}^{\prime}\left(v_{1}\right)^{2}=q_{1}\left(v_{1}\right)$. We have

$$
\begin{aligned}
f_{1}^{\prime}\left(v_{1}\right)^{2}= & \left(f_{1}\left(v_{1}\right)+(n-1) \cdot u_{1}\right)^{2} \\
= & f_{1}\left(v_{1}\right)^{2}+(n-1)^{2} q_{1}\left(u_{1}\right) \cdot 1 \\
& +(n-1) \cdot u_{1}\left(v_{1}+u_{1}\left(u_{3} v_{3}+\cdots+u_{2 n-1} v_{2 n-1}\right)\right) \\
& +(n-1) \cdot\left(v_{1}+u_{1}\left(u_{3} v_{3}+\cdots+u_{2 n-1} v_{2 n-1}\right)\right) u_{1} \\
= & q_{1}\left(v_{1}\right) \cdot 1+(n-1)^{2} \cdot 1+(n-1) \cdot\left(u_{1} v_{1}+v_{1} u_{1}\right) \\
= & q_{1}\left(v_{1}\right) \cdot 1+(n-1) \cdot 1+(n-1) \cdot 1=q_{1}\left(v_{1}\right) \cdot 1 .
\end{aligned}
$$

So the claim is proved. Since the image of $f_{1}^{\prime}$ commutes with that of $f_{2}$, the map $f=f_{1}^{\prime} \oplus f_{2}$ on $W_{1} \perp W_{2}$ is compatible with $q=q_{1} \perp q_{2}$ and it can be extended to an isomorphism $\bar{f}: C(L)=C\left(W_{1} \perp W_{2}\right) \simeq C(L)=C\left(\mathbb{A}_{1} \perp L^{\prime}\right)$ where $L=\mathbb{A}_{1} \perp \cdots \perp \mathbb{A}_{n}$ and $L^{\prime}=\mathbb{A}_{2} \perp \cdots \perp \mathbb{A}_{n}$. By (4.4), there exists an isomorphism $g: C\left(\mathbb{A}_{1} \perp L^{\prime}\right) \simeq C\left(\mathbb{A}_{1}\right) \otimes C\left(L^{\prime}\right)$. So there exists an isomorphism $\psi=g \circ \bar{f}: C(L) \simeq C\left(\mathbb{A}_{1}\right) \otimes C\left(L^{\prime}\right)$. The restriction of $\psi$ to $C_{0}(L)$ maps $C_{0}(L)$ to $C_{0}\left(\mathbb{A}_{1}\right) \otimes C\left(L^{\prime}\right)$. So we have an isomorphism $\psi: C_{0}(L) \simeq C_{0}\left(\mathbb{A}_{1}\right) \otimes C\left(L^{\prime}\right)$.

Proposition 7.5. For $n \geqslant 2$, let $\left(\mathbb{A}_{1}, q_{1}\right), \cdots,\left(\mathbb{A}_{n}, q_{n}\right)$ be 4-dimensional quadratic spaces over a field $F$ of characteristic 2 . For $i=1, \cdots, n$, let $\tau_{i}$ be an interchange isometry of $\mathbb{A}_{i}$. We have $\left(C_{0}\left(\mathbb{A}_{1} \perp \cdots \perp \mathbb{A}_{n}\right), J_{\tau_{1} \perp \cdots \perp \tau_{n}}\right) \simeq$ $\left(C_{0}\left(\mathbb{A}_{1}\right), J_{\tau_{1}}\right) \otimes\left(C\left(\mathbb{A}_{2} \perp \cdots \perp \mathbb{A}_{n}\right), J_{\tau_{2} \perp \cdots \perp \tau_{n}}\right)$.

Proof. By (3.6), for every $1 \leqslant i \leqslant n$, there exist quadratic planes $\mathbb{H}_{2 i-1}$ and $\mathbb{H}_{2 i}$ such that $\mathbb{A}_{i}=\mathbb{H}_{2 i-1} \perp \mathbb{H}_{2 i}, \tau_{i}\left(\mathbb{H}_{2 i-1}\right)=\mathbb{H}_{2 i}$ and $\tau_{i}\left(\mathbb{H}_{2 i}\right)=\mathbb{H}_{2 i-1}$. Let $\left\{u_{i}, v_{i}\right\}, i=1, \cdots, 2 n$, be a standard symplectic basis of $\mathbb{H}_{i} \simeq[1,0]$ such that for $1 \leqslant i \leqslant n, \tau\left(u_{2 i-1}\right)=u_{2 i}, \tau\left(u_{2 i}\right)=u_{2 i-1}, \tau\left(v_{2 i-1}\right)=v_{2 i}$ and $\tau\left(v_{2 i}\right)=v_{2 i-1}$. Set $L=\mathbb{A}_{1} \perp \cdots \perp \mathbb{A}_{n}, L^{\prime}=\mathbb{A}_{2} \perp \cdots \perp \mathbb{A}_{n}, \tau=\tau_{1} \perp \cdots \perp \tau_{n}$ and $\tau^{\prime}=\tau_{2} \perp \cdots \perp \tau_{n}$. Let $\psi: C_{0}(L) \simeq C_{0}\left(\mathbb{A}_{1}\right) \otimes C\left(L^{\prime}\right)$ be the isomorphism discussed in (7.4). We want to show that $\left(J_{\tau_{1}} \otimes J_{\tau^{\prime}}\right) \circ \psi=\psi \circ J_{\tau}$. We have

$$
\left(J_{\tau_{1}} \otimes J_{\tau^{\prime}}\right) \circ \psi\left(u_{1}\right)=\left(J_{\tau_{1}} \otimes J_{\tau^{\prime}}\right)\left(u_{1} \otimes 1\right)=u_{2} \otimes 1=\psi\left(u_{2}\right)=\psi \circ J_{\tau}\left(u_{1}\right),
$$

similarly

$$
\left(J_{\tau_{1}} \otimes J_{\tau^{\prime}}\right) \circ \psi\left(u_{2}\right)=\psi \circ J_{\tau}\left(u_{2}\right) .
$$


We also have

$$
\begin{aligned}
\left(J_{\tau_{1}} \otimes J_{\tau^{\prime}}\right) \circ \psi\left(v_{1}\right)= & \left(J_{\tau_{1}} \otimes J_{\tau^{\prime}}\right)\left((n-1) \cdot u_{1} \otimes 1+v_{1} \otimes 1\right. \\
& \left.+u_{1} \otimes\left(u_{3} v_{3}+\cdots+u_{2 n-1} v_{2 n-1}\right)\right) \\
= & (n-1) \cdot u_{2} \otimes 1+v_{2} \otimes 1+u_{2} \otimes\left(v_{4} u_{4}+\cdots+v_{2 n} u_{2 n}\right) \\
= & (n-1) \cdot u_{2} \otimes 1+v_{2} \otimes 1 \\
& +u_{2} \otimes\left(u_{4} v_{4}+\cdots+u_{2 n} v_{2 n}+(n-1) \cdot 1\right) \\
= & v_{2} \otimes 1+u_{2} \otimes\left(u_{4} v_{4}+\cdots+u_{2 n} v_{2 n}\right)=\psi\left(v_{2}\right)=\psi \circ J_{\tau}\left(v_{1}\right),
\end{aligned}
$$

similarly

$$
\left(J_{\tau_{1}} \otimes J_{\tau^{\prime}}\right) \circ \psi\left(v_{2}\right)=\psi \circ J_{\tau}\left(v_{2}\right) .
$$

For every $2 \leqslant i \leqslant n$, we have

$$
\begin{aligned}
\left(J_{\tau_{1}} \otimes J_{\tau^{\prime}}\right) \circ \psi\left(u_{2 i-1}\right) & =\left(J_{\tau_{1}} \otimes J_{\tau^{\prime}}\right)\left(u_{1} \otimes u_{2 i-1}\right)=u_{2} \otimes u_{2 i} \\
& =\psi\left(u_{2 i}\right)=\psi \circ J_{\tau}\left(u_{2 i-1}\right),
\end{aligned}
$$

similarly

$$
\left(J_{\tau_{1}} \otimes J_{\tau^{\prime}}\right) \circ \psi\left(v_{2 i-1}\right)=\psi \circ J_{\tau}\left(v_{2 i-1}\right) .
$$

Finally for every $2 \leqslant i \leqslant n$, we have

$$
\begin{aligned}
\left(J_{\tau_{1}} \otimes J_{\tau^{\prime}}\right) \circ \psi\left(u_{2 i}\right) & =\left(J_{\tau_{1}} \otimes J_{\tau^{\prime}}\right)\left(u_{2} \otimes u_{2 i}\right)=u_{1} \otimes u_{2 i-1} \\
& =\psi\left(u_{2 i-1}\right)=\psi \circ J_{\tau}\left(u_{2 i}\right),
\end{aligned}
$$

and similarly

$$
\left(J_{\tau_{1}} \otimes J_{\tau^{\prime}}\right) \circ \psi\left(v_{2 i}\right)=\psi \circ J_{\tau}\left(v_{2 i}\right) .
$$

Remark 7.6. Let $(V, q)$ be a quadratic space over a field $F$ of characteristic 2 and let $C(V)$ be the Clifford algebra of $(V, q)$. If $\tau$ is an involution in $O(V, q)$, then $\tau$ induces an involution $J_{\tau}^{\prime q}$ on $C(V) \times C(V)$ such that $J_{\tau}^{\prime q}(v, 0)=(0, \tau(v))$ and $J_{\tau}^{\prime q}(0, v)=(\tau(v), 0)$ for every $v \in V$. We usually denote $J_{\tau}^{\prime q}$ by $J_{\tau}^{\prime}$.

We need the following straightforward result.

Lemma 7.7. Let $(V, q)$ and $\left(V^{\prime}, q^{\prime}\right)$ be two quadratic spaces over a field $F$ of characteristic 2 and let $\tau$ and $\tau^{\prime}$ be involutions in $O(V, q)$ and $O\left(V^{\prime}, q^{\prime}\right)$, respectively. If $\left(C(V), J_{\tau}\right) \simeq\left(C\left(V^{\prime}\right), J_{\tau^{\prime}}\right)$, then $\left(C(V) \times C(V), J_{\tau}^{\prime}\right) \simeq\left(C\left(V^{\prime}\right) \times\right.$ $\left.C\left(V^{\prime}\right), J_{\tau^{\prime}}^{\prime}\right)$.

Proposition 7.8. For $n \geqslant 2$, let $(V, q)$ be a $2 n$-dimensional quadratic space over a field $F$ of characteristic 2 and let $\tau$ be an involution in $O(V, q)$. Suppose that $\Delta(q)$ is trivial and $V=\mathbb{E} \perp V^{\prime}$ where $\mathbb{E}$ is a quadratic subplane of $V$ such that $\tau(\mathbb{E})=\mathbb{E}$ and $\tau\left(V^{\prime}\right)=V^{\prime}$. Let $t=\operatorname{dim} \operatorname{Fix}(V, \tau)-\frac{1}{2} \operatorname{dim} V, \tau^{\prime}=\left.\tau\right|_{V^{\prime}}$ and $u_{1} \in \mathbb{E}$ be an anisotropic vector such that $\tau\left(u_{1}\right)=u_{1}$. Set $a=q\left(u_{1}\right)$.

(a) If $t \equiv 0 \bmod 2$, then $\left(C_{0}(V, q), J_{\tau}\right) \simeq\left(C\left(V^{\prime}, a \cdot q\right) \times C\left(V^{\prime}, a \cdot q\right), J_{\tau^{\prime}}^{a \cdot q} \times J_{\tau^{\prime}}^{a \cdot q}\right)$. 
(b) If $t \equiv 1 \bmod 2$, then $\left(C_{0}(V, q), J_{\tau}\right) \simeq\left(C\left(V^{\prime}, a \cdot q\right) \times C\left(V^{\prime}, a \cdot q\right), J_{\tau^{\prime}}^{\prime a \cdot q}\right)$.

Also $C\left(V^{\prime}, a \cdot q\right)$ is Brauer equivalent to $C(V, q)$. By abuse of notation, we have denoted the form $\left.a \cdot q\right|_{V^{\prime}}$ by $a \cdot q$.

Proof. Let $\left\{u_{2}, v_{2}, \cdots, u_{n}, v_{n}\right\}$ be a symplectic basis of $V^{\prime}$. By (7.3), we can extend $\left\{u_{1}\right\}$ to a symplectic basis $\left\{u_{1}, v_{1}\right\}$ of $\mathbb{E}$ such that $q\left(u_{1}\right) q\left(v_{1}\right)+\cdots+$ $q\left(u_{n}\right) q\left(v_{n}\right)=0$. Let $z=u_{1} v_{1}+\cdots+u_{n} v_{n}$. Then by [4, pp. 122-124], the map $\psi: C\left(V^{\prime}, a \cdot q\right) \times C\left(V^{\prime}, a \cdot q\right) \rightarrow C_{0}(V, q)$ induced by $\psi\left(u_{i}, 0\right)=u_{1} u_{i} z$, $\psi\left(v_{i}, 0\right)=u_{1} v_{i} z, \psi\left(0, u_{i}\right)=u_{1} u_{i}(z+1)$ and $\psi\left(0, v_{i}\right)=u_{1} v_{i}(z+1), i=1, \cdots, n$, is an $F$-algebra isomorphism. Set $\sigma=\psi^{-1} \circ J_{\tau} \circ \psi$. Then $\sigma$ is an involution on $C\left(V^{\prime}, a \cdot q\right) \times C\left(V^{\prime}, a \cdot q\right)$.

(a) We must show that $J_{\tau} \circ \psi=\psi \circ\left(J_{\tau^{\prime}}^{a \cdot q} \times J_{\tau^{\prime}}^{a \cdot q}\right)$ or equivalently $\sigma=$ $J_{\tau^{\prime}}^{a \cdot q} \times J_{\tau^{\prime}}^{a \cdot q}$. For this it suffices to verify that for every $w, w^{\prime} \in C\left(V^{\prime}\right)$ we have $\sigma\left(w, w^{\prime}\right)=\left(\tau^{\prime}(w), \tau^{\prime}\left(w^{\prime}\right)\right)$. It is enough to check this for $\left(w, w^{\prime}\right)=(x, 0)$ and $\left(w, w^{\prime}\right)=(0, x)$ where $x \in V^{\prime}$. By $(4.12)$, we have $\tau(z)=z$. As $z \in Z\left(C_{0}(V)\right)$, we have $z w=w z$ for every $w \in C_{0}(V)$. Also $u_{1} x=x u_{1}$ for every $x \in V^{\prime}$. By the hypothesis, for every $x \in V^{\prime}$ we have $\tau(x) \in V^{\prime}$ and

$$
\begin{aligned}
\sigma(x, 0) & =\psi^{-1} \circ J_{\tau} \circ \psi(x, 0)=\psi^{-1} \circ J_{\tau}\left(u_{1} x z\right)=\psi^{-1}\left(z \tau(x) u_{1}\right) \\
& =\psi^{-1}\left(u_{1} \tau(x) z\right)=(\tau(x), 0)=\left(\tau^{\prime}(x), 0\right) .
\end{aligned}
$$

Similarly

$$
\begin{aligned}
\sigma(0, x) & =\psi^{-1} \circ J_{\tau} \circ \psi(0, x)=\psi^{-1} \circ J_{\tau}\left(u_{1} x(z+1)\right)=\psi^{-1}\left((z+1) \tau(x) u_{1}\right) \\
& =\psi^{-1}\left(u_{1} \tau(x)(z+1)\right)=(0, \tau(x))=\left(0, \tau^{\prime}(x)\right)
\end{aligned}
$$

So $\left(C_{0}(V, q), J_{\tau}\right) \simeq\left(C\left(V^{\prime}, a \cdot q\right) \times C\left(V^{\prime}, a \cdot q\right), J_{\tau^{\prime}}^{a \cdot q} \times J_{\tau^{\prime}}^{a \cdot q}\right)$.

(b) Like in the previous case, it suffices to verify that for every $w, w^{\prime} \in C\left(V^{\prime}\right)$ we have $\sigma\left(w, w^{\prime}\right)=\left(\tau^{\prime}\left(w^{\prime}\right), \tau^{\prime}(w)\right)$. It is enough to check this for $\left(w, w^{\prime}\right)=(x, 0)$ and $\left(w, w^{\prime}\right)=(0, x)$ where $x \in V^{\prime}$. By (4.12), we have $\tau(z)=z+1$. Again by the hypothesis, for every $x \in V^{\prime}$ we have $\tau(x) \in V^{\prime}$ and

$$
\begin{aligned}
\sigma(x, 0) & =\psi^{-1} \circ J_{\tau} \circ \psi(x, 0)=\psi^{-1} \circ J_{\tau}\left(u_{1} x z\right)=\psi^{-1}\left((z+1) \tau(x) u_{1}\right) \\
& =\psi^{-1}\left(u_{1} \tau(x)(z+1)\right)=(0, \tau(x))=\left(0, \tau^{\prime}(x)\right) .
\end{aligned}
$$

Similarly

$$
\begin{aligned}
\sigma(0, x) & =\psi^{-1} \circ J_{\tau} \circ \psi(0, x)=\psi^{-1} \circ J_{\tau}\left(u_{1} x(z+1)\right)=\psi^{-1}\left(z \tau(x) u_{1}\right) \\
& =\psi^{-1}\left(u_{1} \tau(x) z\right)=(\tau(x), 0)=\left(\tau^{\prime}(x), 0\right) .
\end{aligned}
$$

So $\left(C_{0}(V, q), J_{\tau}\right) \simeq\left(C\left(V^{\prime}, a \cdot q\right) \times C\left(V^{\prime}, a \cdot q\right), J_{\tau^{\prime}}^{\prime a \cdot q}\right)$.

The last statement follows from $(5.1$ (i)) and the triviality of the Arf invariant.

Proposition 7.9. Let $(V, q)$ be a $2 n$-dimensional quadratic space over a field $F$ of characteristic 2 and let $\tau$ be an involution in $O(V, q)$. Then there exists a quadratic space $\left(V^{\prime}, q^{\prime}\right)$ such that $\left(C(V) \times C(V), J_{\tau}^{\prime}\right) \simeq\left(C\left(V^{\prime}\right) \times C\left(V^{\prime}\right), J_{\text {id }}^{\prime}\right)$.

Proof. If $J_{\tau}$ is of symplectic type, then by (6.3), there exists a quadratic space $\left(V^{\prime}, q^{\prime}\right)$ over $F$ such that $\left(C(V), J_{\tau}\right) \simeq\left(C\left(V^{\prime}\right), J_{\text {id }}\right)$, so by $(7.7)$, we have $(C(V) \times$ 
$\left.C(V), J_{\tau}^{\prime}\right) \simeq\left(C\left(V^{\prime}\right) \times C\left(V^{\prime}\right), J_{\text {id }}^{\prime}\right)$. So consider the case where $J_{\tau}$ is of orthogonal type, thus by (4.7), $\tau$ is either an orthogonal sum of reflections or an orthogonal sum of interchange isometries. By (6.10), the Clifford algebra of a 4-dimensional quadratic space with an involution induced by an interchange isometry is isomorphic to the Clifford algebra of a 4-dimensional quadratic space with an involution induced by an orthogonal sum of two reflections, so in order to prove the result we may consider the case where $\tau=\tau_{1} \perp \cdots \perp \tau_{n}$ is an orthogonal sum of reflections. Let $V=\mathbb{E}_{1} \perp \cdots \perp \mathbb{E}_{n}$ be a Wiitala decomposition of $(V, \tau)$ such that $\tau_{i}=\tau||_{\mathbb{E}_{i}}$. Let $\left\{u_{1}, v_{1}, \cdots, u_{n}, v_{n}\right\}$ be a $\tau$-symplectic basis of $V$ and $\delta=q\left(u_{1}\right) q\left(v_{1}\right)+\cdots+q\left(u_{n}\right) q\left(v_{n}\right) \in F$. Let $\left(\mathbb{E}_{n+1}, \varphi\right)$ be the quadratic plane $[1, \delta]$ over $F$ with a standard symplectic basis $\left\{u_{n+1}, v_{n+1}\right\}$ and set $z=$ $u_{1} v_{1}+\cdots+u_{n+1} v_{n+1} \in C\left(V \perp \mathbb{E}_{n+1}\right)$. Consider the involution $\rho=\tau \perp \mathrm{id}_{\mathbb{E}_{n+1}} \in$ $O\left(V \perp \mathbb{E}_{n+1}, q \perp \varphi\right)$. We have $\operatorname{dim} \operatorname{Fix}\left(V \perp \mathbb{E}_{n+1}, \rho\right)-\frac{1}{2} \operatorname{dim}\left(V \perp \mathbb{E}_{n+1}\right)=$ $(n+2)-(n+1)=1$ and $q\left(u_{1}\right) q\left(v_{1}\right)+\cdots+q\left(u_{n+1}\right) q\left(v_{n+1}\right)=0$, so by $(7.8(\mathrm{~b}))$, we have $\left(C_{0}\left(V \perp \mathbb{E}_{n+1}\right), J_{\rho}\right) \simeq\left(C(V) \times C(V), J_{\tau}^{\prime}\right)$; note that $a=\varphi\left(u_{n+1}\right)=1$. On the other hand $V \perp \mathbb{E}_{n+1}=\mathbb{E}_{1} \perp V_{1}$ where $V_{1}=\mathbb{E}_{2} \perp \cdots \perp \mathbb{E}_{n+1}$. Again by $(7.8(\mathrm{~b}))$, we have $\left(C_{0}\left(V \perp \mathbb{E}_{n+1}\right), J_{\rho}\right) \simeq\left(C_{0}\left(\mathbb{E}_{1} \perp V_{1}\right), J_{\tau_{1} \perp \rho_{1}}\right) \simeq\left(C\left(V_{1}, a \cdot q \perp\right.\right.$ $\left.a \cdot \varphi) \times C\left(V_{1}, a \cdot q \perp a \cdot \varphi\right), J_{\rho_{1}}^{\prime}\right)$ where $\rho_{1}=\tau_{2} \perp \cdots \perp \tau_{n} \perp \operatorname{id}_{\mathbb{E}_{n+1}}$ and $a=q\left(u_{1}\right) \neq 0$. By (4.7), the involution $J_{\rho_{1}}$ on $C\left(V_{1}, a \cdot q \perp a \cdot \varphi\right)$ is of symplectic type, so by (6.3), we may write $\left(C\left(V_{1}, a \cdot q \perp a \cdot \varphi\right), J_{\rho_{1}}\right) \simeq\left(C\left(V^{\prime}, q^{\prime}\right), J_{\text {id }}\right)$ for a suitable quadratic space $\left(V^{\prime}, q^{\prime}\right)$. So by $(7.7),\left(C\left(V_{1}, a \cdot q \perp a \cdot \varphi\right) \times C\left(V_{1}, a \cdot q \perp\right.\right.$ $\left.a \cdot \varphi), J_{\rho_{1}}^{\prime}\right) \simeq\left(C\left(V^{\prime}\right) \times C\left(V^{\prime}\right), J_{\text {id }}^{\prime}\right)$. Therefore, $\left(C(V) \times C(V), J_{\tau}^{\prime}\right) \simeq\left(C\left(V^{\prime}\right) \times\right.$ $\left.C\left(V^{\prime}\right), J_{\text {id }}^{\prime}\right)$ and the result is proved.

Lemma 7.10. Let $(\mathbb{A}, q)$ be a 4-dimensional quadratic space over a field $F$ of characteristic 2 and let $\tau$ be an interchange isometry of $(\mathbb{A}, q)$. Then $\left(C_{0}(\mathbb{A}), J_{\tau}\right)$ $\simeq\left(C(\mathbb{H}), J_{\tau_{u}}\right) \times\left(C(\mathbb{H}), J_{\mathrm{id}}\right)$ where $\mathbb{H}$ is a hyperbolic subplane of $\mathbb{A}$ and $u$ is a suitable anisotropic element of $\mathbb{H}$.

Proof. By (3.6), there exist a decomposition $\mathbb{A}=\mathbb{H}_{1} \perp \mathbb{H}_{2}$ of $\mathbb{A}$ into hyperbolic planes $\mathbb{H}_{1}$ and $\mathbb{H}_{2}$ over $F$ and standard symplectic bases $\left\{u_{i}, v_{i}\right\}$ of $\mathbb{H}_{i} \simeq[1,0]$, $i=1,2$, such that $\tau\left(u_{1}\right)=u_{2}, \tau\left(u_{2}\right)=u_{1}, \tau\left(v_{1}\right)=v_{2}$ and $\tau\left(v_{2}\right)=v_{1}$. We have $q\left(u_{1}\right) q\left(v_{1}\right)+q\left(u_{2}\right) q\left(v_{2}\right)=0$. Set $z=u_{1} v_{1}+u_{2} v_{2} \in C_{0}(\mathbb{A})$. By (4.12), we have $z^{2}=z$ and $\tau(z)=z$. Also by $(7.8)$, the map $\psi: C\left(\mathbb{H}_{2}\right) \times C\left(\mathbb{H}_{2}\right) \rightarrow C_{0}(\mathbb{A})$ induced by $\psi\left(u_{2}, 0\right)=u_{1} u_{2} z, \psi\left(v_{2}, 0\right)=u_{1} v_{2} z, \psi\left(0, u_{2}\right)=u_{1} u_{2}(z+1)$ and $\psi\left(0, v_{2}\right)=u_{1} v_{2}(z+1)$ is an isomorphism. Set $\sigma=\psi^{-1} \circ J_{\tau} \circ \psi$. Then $\sigma$ is an involution on $C\left(\mathbb{H}_{2}\right) \times C\left(\mathbb{H}_{2}\right)$. We claim that $\sigma(x, y)=\left(J_{\tau_{u_{2}}}(x), J_{\mathrm{id}}(y)\right)$ for every $x, y \in C\left(\mathbb{H}_{2}\right)$. It is enough to check this equality for $(x, y)=\left(u_{2}, 0\right)$, $\left(v_{2}, 0\right),\left(0, u_{2}\right)$ and $\left(0, v_{2}\right)$. We have

$$
\begin{aligned}
\sigma\left(u_{2}, 0\right) & =\psi^{-1} \circ J_{\tau} \circ \psi\left(u_{2}, 0\right)=\psi^{-1} \circ J_{\tau}\left(u_{1} u_{2} z\right)=\psi^{-1}\left(z u_{1} u_{2}\right) \\
& =\psi^{-1}\left(u_{1} u_{2} z\right)=\left(u_{2}, 0\right)=\left(J_{\tau_{u_{2}}}\left(u_{2}\right), 0\right),
\end{aligned}
$$

Note that we have $u_{1} z=u_{1}\left(u_{1} v_{1}+u_{2} v_{2}\right)=v_{1}+u_{1} u_{2} v_{2}$, so $v_{1}=u_{1} z+u_{1} u_{2} v_{2}$ and we obtain

$$
\begin{aligned}
\sigma\left(v_{2}, 0\right) & =\psi^{-1} \circ J_{\tau} \circ \psi\left(v_{2}, 0\right)=\psi^{-1} \circ J_{\tau}\left(u_{1} v_{2} z\right)=\psi^{-1}\left(z v_{1} u_{2}\right) \\
& =\psi^{-1}\left(u_{2} v_{1} z\right)=\psi^{-1}\left(u_{2}\left(u_{1} z+u_{1} u_{2} v_{2}\right) z\right)=\psi^{-1}\left(u_{1} u_{2} z+u_{1} v_{2} z\right) \\
& =\left(u_{2}+v_{2}, 0\right)=\left(v_{2}+\frac{B\left(u_{2}, v_{2}\right)}{q\left(u_{2}\right)} u_{2}, 0\right)=\left(J_{\tau_{u_{2}}}\left(v_{2}\right), 0\right) .
\end{aligned}
$$


Similarly we have

$$
\begin{aligned}
\sigma\left(0, u_{2}\right) & =\psi^{-1} \circ J_{\tau} \circ \psi\left(0, u_{2}\right)=\psi^{-1} \circ J_{\tau}\left(u_{1} u_{2}(z+1)\right) \\
& =\psi^{-1}\left((z+1) u_{1} u_{2}\right)=\psi^{-1}\left(u_{1} u_{2}(z+1)\right)=\left(0, u_{2}\right)=\left(0, J_{\text {id }}\left(u_{2}\right)\right),
\end{aligned}
$$

Finally

$$
\begin{aligned}
\sigma\left(0, v_{2}\right) & =\psi^{-1} \circ J_{\tau} \circ \psi\left(0, v_{2}\right)=\psi^{-1} \circ J_{\tau}\left(u_{1} v_{2}(z+1)\right)=\psi^{-1}\left((z+1) v_{1} u_{2}\right) \\
& =\psi^{-1}\left(u_{2} v_{1}(z+1)\right)=\psi^{-1}\left(u_{2}\left(u_{1} z+u_{1} u_{2} v_{2}\right)(z+1)\right) \\
& =\psi^{-1}\left(0+u_{1} v_{2}(z+1)\right)=\left(0, v_{2}\right)=\left(0, J_{\mathrm{id}}\left(v_{2}\right)\right)
\end{aligned}
$$

Theorem 7.11. Let $(V, q)$ be a $2 n$-dimensional quadratic space over a field $F$ of characteristic 2 and let $\tau$ be an involution in $O(V, q)$. Suppose that $\Delta(q)$ is trivial and let $t=\operatorname{dim} \operatorname{Fix}(V, \tau)-\frac{1}{2} \operatorname{dim} V$.

(a) If $t=0$ and $\tau$ is of reflectional kind, then there exists a $(2 n-2)$-dimensional quadratic space $\left(V^{\prime}, q^{\prime}\right)$ over $F$ such that $\left(C_{0}(V), J_{\tau}\right) \simeq\left(C\left(V^{\prime}\right) \times C\left(V^{\prime}\right), J_{\tau^{\prime}} \times\right.$ $\left.J_{\tau^{\prime}}\right)$ where $\tau^{\prime}$ is an involution in $O\left(V^{\prime}, q^{\prime}\right)$ which is an orthogonal sum of $n-1$ reflections. More precisely $\left(V^{\prime}, q^{\prime}\right)$ is similar to a subform of $(V, q)$ which can be explicitly described by (7.8).

(b) If $t=0$ and $\tau$ is of interchanging kind, then there exist $(2 n-2)$-dimensional quadratic spaces $\left(V^{\prime}, q^{\prime}\right)$ and $\left(V^{\prime \prime}, q^{\prime \prime}\right)$ over $F$ such that $\left(C_{0}(V), J_{\tau}\right) \simeq$ $\left(C\left(V^{\prime}\right) \times C\left(V^{\prime \prime}\right), J_{\tau^{\prime}} \times J_{\text {id }}\right)$ where $\tau^{\prime}$ is an involution in $O\left(V^{\prime}, q^{\prime}\right)$ which is an orthogonal sum of $n-1$ reflections. Furthermore we have $C\left(V^{\prime}\right) \simeq C\left(V^{\prime \prime}\right)$ and $C\left(V^{\prime}\right)$ is isomorphic to the Clifford algebra of a hyperbolic space over F.

(c) If $0 \neq t \equiv 0 \bmod 2$, then there exists a $(2 n-2)$-dimensional quadratic space $\left(V^{\prime}, q^{\prime}\right)$ over $F$ such that $\left(C_{0}(V), J_{\tau}\right) \simeq\left(C\left(V^{\prime}\right), J_{\mathrm{id}}\right) \times\left(C\left(V^{\prime}\right), J_{\mathrm{id}}\right)$.

(d) If $t \equiv 1 \bmod 2$, then there exists a $(2 n-2)$-dimensional quadratic space $\left(V^{\prime}, q^{\prime}\right)$ over $F$ such that $\left(C_{0}(V), J_{\tau}\right) \simeq\left(C\left(V^{\prime}\right) \times C\left(V^{\prime}\right), J_{\text {id }}^{\prime}\right)$.

Proof. Let $V=W \perp \mathbb{E}_{1} \perp \cdots \perp \mathbb{E}_{r} \perp \mathbb{A}_{1} \perp \cdots \perp \mathbb{A}_{s}$ be a Wiitala decomposition of $(V, \tau)$ where $W$ is a maximal fixed orthogonal summand of $(V, \tau)$. Let $\left\{u_{1}, v_{1}, \cdots, u_{n}, v_{n}\right\}$ be a $\tau$-symplectic basis of $V$ with respect to this decomposition. By (3.19), we have $t=\frac{1}{2} \operatorname{dim} W$. Set $z=u_{1} v_{1}+\cdots+u_{n} v_{n} \in C_{0}(V)$. By (4.12), $J_{\tau}(z)=z+t \cdot 1$.

(a) We have $W=0$ and $J_{\tau}(z)=z$. As $\tau$ is of reflectional kind, we may assume that $s=0$ and $r=n$, so one can write $\tau=\tau_{u_{1}} \perp \cdots \perp \tau_{u_{n}}$ where $\tau_{u_{i}}$ is the reflection along the anisotropic vector $u_{i} \in \mathbb{E}_{i}$. Let $V^{\prime}=\mathbb{E}_{2} \perp \cdots \perp \mathbb{E}_{n}$ and $a=q\left(u_{1}\right)$. By $(7.8(\mathrm{a})),\left(C_{0}(V), J_{\tau}\right) \simeq\left(C\left(V^{\prime}, a \cdot q\right) \times C\left(V^{\prime}, a \cdot q\right), J_{\tau^{\prime}} \times J_{\tau^{\prime}}\right)$ where $\tau^{\prime}=\tau_{u_{2}} \perp \cdots \perp \tau_{u_{n}}$.

(b) We have $W=0$ and $J_{\tau}(z)=z$. As $\tau$ is of interchanging kind, we may assume that $r=0, n=2 s$ and $\tau=\tau_{1} \perp \cdots \perp \tau_{s}$ where $\tau_{i}$ is an interchange isometry of $\mathbb{A}_{i}, i=1, \cdots, s$. If $n=2$, by $(7.10)$, we have $\left(C_{0}(V), J_{\tau}\right) \simeq(C(\mathbb{H}) \times$ $\left.C(\mathbb{H}), J_{\tau^{\prime}} \times J_{\text {id }}\right)$ where $\mathbb{H}$ is a hyperbolic subplane of $V$ and $\tau^{\prime}$ is a reflection of 
IH. If $n \geqslant 3$, by $(7.5)$, we have $\left(C_{0}(V), J_{\tau_{1} \perp \cdots \perp \tau_{s}}\right) \simeq\left(C_{0}\left(\mathbb{A}_{1}\right), J_{\tau_{1}}\right) \otimes\left(C\left(\mathbb{A}_{2} \perp\right.\right.$ $\left.\left.\cdots \perp \mathbb{A}_{s}\right), J_{\tau_{2} \perp \cdots \perp \tau_{s}}\right)$. Using (7.5), (7.10) and (7.2) we obtain

$$
\begin{aligned}
\left(C_{0}(V), J_{\tau_{1} \perp \cdots \perp \tau_{s}}\right) \simeq & \left(C_{0}\left(\mathbb{A}_{1}\right), J_{\tau_{1}}\right) \otimes\left(C\left(\mathbb{A}_{2} \perp \cdots \perp \mathbb{A}_{s}\right), J_{\tau_{2} \perp \cdots \perp \tau_{s}}\right) \\
\simeq & \left(\left(C\left(\mathbb{H}_{1}\right), J_{\tau_{1}^{\prime}}\right) \times\left(C\left(\mathbb{H}_{1}\right), J_{\mathrm{id}}\right)\right) \\
& \otimes\left(C\left(\mathbb{A}_{2} \perp \cdots \perp \mathbb{A}_{s}\right), J_{\tau_{2} \perp \cdots \perp \tau_{s}}\right) \\
\simeq & \left(\left(C\left(\mathbb{H}_{1}\right), J_{\tau_{1}^{\prime}}\right) \otimes\left(C\left(\mathbb{A}_{2} \perp \cdots \perp \mathbb{A}_{s}\right), J_{\tau_{2} \perp \cdots \perp \tau_{s}}\right)\right) \\
& \times\left(\left(C\left(\mathbb{H}_{1}\right), J_{\mathrm{id}}\right) \otimes\left(C\left(\mathbb{A}_{2} \perp \cdots \perp \mathbb{A}_{s}\right), J_{\tau_{2} \perp \cdots \perp \tau_{s}}\right)\right),
\end{aligned}
$$

where $\mathbb{H}_{1}$ is a hyperbolic subplane of $\mathbb{A}_{1}$ and $\tau_{1}^{\prime}$ is a reflection of $\mathbb{H}_{1}$. By (6.10), we have $\left(C\left(\mathbb{A}_{2} \perp \cdots \perp \mathbb{A}_{s}\right), J_{\tau_{2} \perp \cdots \perp \tau_{s}}\right) \simeq\left(C(L), J_{\rho}\right)$, where $L=\mathbb{H}_{2} \perp \cdots \perp$ $\mathbb{H}_{2 s-1}$ is an orthogonal sum of $2 s-2=n-2$ hyperbolic planes $\mathbb{H}_{i}$ over $F$ and $\rho=\tau_{2}^{\prime} \perp \cdots \perp \tau_{2 s-1}^{\prime}$ is an orthogonal sum of $2 s-2$ reflections $\tau_{i}^{\prime}$ of $\mathbb{H}_{i}$. So we obtain

$$
\begin{aligned}
\left(C_{0}(V), J_{\tau_{1} \perp \cdots \perp \tau_{s}}\right) & \simeq\left(\left(C\left(\mathbb{H}_{1}\right), J_{\tau_{1}^{\prime}}\right) \otimes\left(C(L), J_{\rho}\right)\right) \times\left(\left(C\left(\mathbb{H}_{1}\right), J_{\mathrm{id}}\right) \otimes\left(C(L), J_{\rho}\right)\right) \\
& \simeq\left(C\left(\mathbb{H}_{1} \perp L\right), J_{\tau_{1}^{\prime} \perp \rho}\right) \times\left(C\left(\mathbb{H}_{1} \perp L\right), J_{\mathrm{id} \perp \rho}\right) .
\end{aligned}
$$

By (4.7), $\left(C\left(\mathbb{H}_{1} \perp L\right), J_{\mathrm{id} \perp \rho}\right)$ is of symplectic type, so by $(6.3)$, there exists a quadratic space $\left(V^{\prime \prime}, q^{\prime \prime}\right)$ over $F$ such that $\left(C\left(\mathbb{E}_{1}^{\prime} \perp L\right), J_{\mathrm{id} \perp \rho}\right) \simeq\left(C\left(V^{\prime \prime}\right), J_{\mathrm{id}}\right)$, so (4) leads to $\left(C_{0}(V), J_{\tau}\right) \simeq\left(C\left(V^{\prime}\right) \times C\left(V^{\prime \prime}\right), J_{\tau^{\prime}} \times J_{\text {id }}\right)$, where $V^{\prime}=\mathbb{H}_{1} \perp L$ and $\tau^{\prime}=\tau_{1}^{\prime} \perp \rho=\tau_{1}^{\prime} \perp \cdots \perp \tau_{2 s-1}^{\prime}$. Finally note that as $L$ is a hyperbolic space, $V^{\prime}$ is also a hyperbolic space, so $C\left(V^{\prime}\right) \simeq C\left(V^{\prime \prime}\right)$ is isomorphic to the Clifford algebra of a hyperbolic space over $F$.

(c) We have $\operatorname{dim} W \geqslant 4$ and $J_{\tau}(z)=z$. So we may assume that for the vector space $W_{1}=F u_{1}+F v_{1}+F u_{2}+F v_{2}$ we have $\left.\tau\right|_{W_{1}}=\operatorname{id}_{W_{1}}$ where $a=q\left(u_{1}\right) \neq 0$. Let $\mathbb{E}=F u_{1}+F v_{1}, V_{1}=F u_{2}+F v_{2}+\cdots+F u_{n}+F v_{n}$ and $\tau^{\prime}=\left.\tau\right|_{V_{1}}$. By $(7.8(\mathrm{a})),\left(C_{0}(V), J_{\tau}\right) \simeq\left(C\left(V_{1}, a \cdot q\right) \times C\left(V_{1}, a \cdot q\right), J_{\tau^{\prime}} \times J_{\tau^{\prime}}\right)$. On the other hand as $\operatorname{dim} \operatorname{Fix}\left(V_{1},\left.\tau\right|_{V_{1}}\right)-\frac{1}{2} \operatorname{dim} V_{1}=t-1 \geqslant 1$, by (4.7), the involution $J_{\tau^{\prime}}$ is of symplectic type, so by $(6.3)$, we have $\left(C\left(V_{1}\right), J_{\tau^{\prime}}\right) \simeq\left(C\left(V^{\prime}\right), J_{\text {id }}\right)$ for some quadratic space $\left(V^{\prime}, q^{\prime}\right)$ over $F$, so $\left(C_{0}(V), J_{\tau}\right) \simeq\left(C\left(V^{\prime}\right) \times C\left(V^{\prime}\right), J_{\text {id }} \times J_{\text {id }}\right)$.

(d) If $t=1$, then $\operatorname{dim} W=2$ and we have $V=W \perp W^{\prime}$ where $W^{\prime}=W^{\perp}$. Let $u \in W$ be an anisotropic vector and $a=q(u)$. By $(7.8(\mathrm{~b}))$ we have $\left(C_{0}(V, q), J_{\tau}\right) \simeq\left(C_{0}\left(W \perp W^{\prime}\right), J_{\mathrm{id}_{W} \perp \tau^{\prime}}\right) \simeq\left(C\left(W^{\prime}, a \cdot q\right) \times C\left(W^{\prime}, a \cdot q\right), J_{\tau^{\prime}}^{\prime}\right)$ where $\tau^{\prime}=\left.\tau\right|_{W^{\prime}}$ and by (6.3) and (7.7), $\left(C\left(W^{\prime}, a \cdot q\right) \times C\left(W^{\prime}, a \cdot q\right), J_{\tau^{\prime}}^{\prime}\right) \simeq$ $\left(C\left(V^{\prime}\right) \times C\left(V^{\prime}\right), J_{\mathrm{id}_{V^{\prime}}}^{\prime}\right)$ for some quadratic space $\left(V^{\prime}, q^{\prime}\right)$. So $\left(C_{0}(V, q), J_{\tau}\right) \simeq$ $\left(C\left(V^{\prime}\right) \times C\left(V^{\prime}\right), J_{\mathrm{id}_{V^{\prime}}^{\prime}}^{\prime}\right)$.

Lemma 7.12. For $i=1,2$, let $\left(A_{i}, \sigma_{i}\right)$ and $\left(B_{i}, \tau_{i}\right)$ be central simple algebras with involution over a field $F$. If $\left(A_{1} \times A_{2}, \sigma_{1} \times \sigma_{2}\right) \simeq\left(B_{1} \times B_{2}, \tau_{1} \times \tau_{2}\right)$, then either $\left(A_{1}, \sigma_{1}\right) \simeq\left(B_{1}, \tau_{1}\right)$ and $\left(A_{2}, \sigma_{2}\right) \simeq\left(B_{2}, \tau_{2}\right)$ or $\left(A_{1}, \sigma_{1}\right) \simeq\left(B_{2}, \tau_{2}\right)$ and $\left(A_{2}, \sigma_{2}\right) \simeq\left(B_{1}, \tau_{1}\right)$.

Proof. Let $\psi:\left(A_{1} \times A_{2}, \sigma_{1} \times \sigma_{2}\right) \simeq\left(B_{1} \times B_{2}, \tau_{1} \times \tau_{2}\right)$ be an isomorphism. The set $B_{1} \times\{0\}$ is a two sided ideal of $B_{1} \times B_{2}$, so $\psi^{-1}\left(B_{1} \times\{0\}\right)$ is a two sided ideal of $A_{1} \times A_{2}$. As $A_{1}$ and $A_{2}$ are simple rings and $\psi$ is an isomorphism, we have either $\psi^{-1}\left(B_{1} \times\{0\}\right)=A_{1} \times\{0\}$ or $\psi^{-1}\left(B_{1} \times\{0\}\right)=\{0\} \times A_{2}$. Suppose that the first case occurs. Define a map $f: A_{1} \rightarrow B_{1}$ as follows: for every $a_{1} \in A_{1}$, there exists an element $b_{1} \in B_{1}$ such that $\psi\left(a_{1}, 0\right)=\left(b_{1}, 0\right)$. Set $f\left(a_{1}\right)=b_{1}$, i.e., for every $a_{1} \in A_{1}$ we have $\psi\left(a_{1}, 0\right)=\left(f\left(a_{1}\right), 0\right)$. As $\psi: A_{1} \times\{0\} \rightarrow B_{1} \times\{0\}$ is 
an isomorphism, the map $f$ is an isomorphism of $F$-algebras. For every $a_{1} \in A_{1}$ we have $\left(\tau_{1} \times \tau_{2}\right) \circ \psi\left(a_{1}, 0\right)=\psi \circ\left(\sigma_{1} \times \sigma_{2}\right)\left(a_{1}, 0\right)$, so $\left(\tau_{1} \times \tau_{2}\right)\left(f\left(a_{1}\right), 0\right)=$ $\psi\left(\sigma_{1}\left(a_{1}\right), 0\right)$. From this we obtain $\left(\tau_{1}\left(f\left(a_{1}\right)\right), 0\right)=\left(f\left(\sigma_{1}\left(a_{1}\right)\right), 0\right)$ which means that $\tau_{1} \circ f=f \circ \sigma_{1}$. So we have proved that $\left(A_{1}, \sigma_{1}\right) \simeq\left(B_{1}, \tau_{1}\right)$. Similarly $\left(A_{2}, \sigma_{2}\right) \simeq\left(B_{2}, \tau_{2}\right)$.

If the second case occurs, a similar argument shows that $\left(A_{1}, \sigma_{1}\right) \simeq\left(B_{2}, \tau_{2}\right)$ and $\left(A_{2}, \sigma_{2}\right) \simeq\left(B_{1}, \tau_{1}\right)$ which completes the proof.

Corollary 7.13. For $i=1, \cdots, n$, let $\left(A_{i}, \sigma_{i}\right)$ and $\left(B_{i}, \tau_{i}\right)$ be central simple algebras with involution over a field $F$. If $\left(A_{1} \times \cdots \times A_{n}, \sigma_{1} \times \cdots \times \sigma_{n}\right) \simeq$ $\left(B_{1} \times \cdots \times B_{n}, \tau_{1} \times \cdots \times \tau_{n}\right)$, then there exists a permutation $\pi \in S_{n}$ such that for every $1 \leqslant i \leqslant n,\left(A_{i}, \sigma_{i}\right) \simeq\left(B_{\pi(i)}, \tau_{\pi(i)}\right)$.

Remark 7.14. The isomorphisms of $(7.11(a))$ and $(7.11(b))$ are the best possible, in the sense that the involution $\tau^{\prime} \in O\left(V^{\prime}, q^{\prime}\right)$ in $(7.11(a))$ and $(7.11(b))$ can not be chosen to be identity; because by $(4.7),\left(C\left(V^{\prime}\right), J_{\tau^{\prime}}\right)$ is of orthogonal type and $\left(C\left(V^{\prime}\right), J_{\text {id }}\right)$ is an algebra with involution of symplectic type. Now by (7.12), the involution $J_{\tau^{\prime}}$ must be of orthogonal type, so $\tau^{\prime}$ can not be chosen to be identity.

In (7.11), we have shown that if $\tau \in O(V, q)$ is an involution and $\Delta(q)=0$, then $\left(C_{0}(V), J_{\tau}\right)$ can be decomposed as a direct product of two isomorphic Clifford algebras with involution. Conversely in (7.15) we will express a direct product of two isomorphic Clifford algebras with involution in the form of $\left(C_{0}(V), J_{\tau}\right)$ for a suitable quadratic space $(V, q)$ with trivial Arf invariant and an involution $\tau \in O(V, q)$. The proof is very similar to that of (7.11).

Remark 7.15. For $i=1,2$, let $\left(V_{i}, q_{i}\right)$ be a $2 n$-dimensional quadratic space over a field $F$ of characteristic 2 . For $i=1,2$, let $\tau_{i}$ be an involution in $O\left(V_{i}, q_{i}\right)$ and set $t_{i}=\operatorname{dim} \operatorname{Fix}\left(V_{i}, \tau_{i}\right)-\frac{1}{2} \operatorname{dim} V_{i}$. Then there exists a $(2 n+2)$-dimensional quadratic space $(V, q)$ over $F$ with trivial Arf invariant such that $\left(C\left(V_{1}\right) \times\right.$ $\left.C\left(V_{2}\right), J_{\tau_{1}} \times J_{\tau_{2}}\right) \simeq\left(C_{0}(V), J_{\tau}\right)$ if and only if one of the following conditions is satisfied:

(a) $t_{1}=t_{2}=0$ and $\left(C\left(V_{1}\right), J_{\tau_{1}}\right) \simeq\left(C\left(V_{2}\right), J_{\tau_{2}}\right)$. In this case $\tau$ is necessarily an orthogonal sum of reflections. In particular $\operatorname{dim} \operatorname{Fix}(V, \tau)=\frac{1}{2} \operatorname{dim} V$, so by $(4.7), J_{\tau}$ is necessarily of orthogonal type.

(b) $t_{1}=0$ and $0 \neq t_{2} \equiv 0 \bmod 2\left(\right.$ resp. $t_{2}=0$ and $\left.0 \neq t_{1} \equiv 0 \bmod 2\right), n \equiv 1$ $\bmod 2, C\left(V_{1}\right) \simeq C\left(V_{2}\right)$ and for every $u \in \operatorname{Fix}\left(V_{1}, \tau_{1}\right)$, we have $q(u) \in F^{2}$. In this case $\tau$ is necessarily an orthogonal sum of interchange isometries. In particular $\operatorname{dim} \operatorname{Fix}(V, \tau)=\frac{1}{2} \operatorname{dim} V$, so by $(4.7), J_{\tau}$ is necessarily of orthogonal type.

(c) $0 \neq t_{i} \equiv 0 \bmod 2, i=1,2$, and $\left(C\left(V_{1}\right), J_{\tau_{1}}\right) \simeq\left(C\left(V_{2}\right), J_{\tau_{2}}\right)$. In this case $\tau$ has necessarily nontrivial maximal fixed orthogonal summand, i.e., $\operatorname{dim} \operatorname{Fix}(V, \tau)>\frac{1}{2} \operatorname{dim} V$, so by $(4.7), J_{\tau}$ is necessarily of symplectic type.

Lemma 7.16. Let $\left(\mathbb{E}_{1}, \varphi_{1}\right)$ and $\left(\mathbb{E}_{2}, \varphi_{2}\right)$ be two quadratic planes over a field $F$ of characteristic 2. Let $\tau_{i}$ be a reflection of $\mathbb{E}_{i}$ and let $\tau_{i}^{\prime}$ be an involution in $O\left(\mathbb{E}_{i}, \varphi_{i}\right)$ where either $\left(\tau_{1}^{\prime}, \tau_{2}^{\prime}\right)=\left(\tau_{1}, \tau_{2}\right)$ or $\left(\tau_{1}^{\prime}, \tau_{2}^{\prime}\right)=(\mathrm{id}, \mathrm{id})$. If $\left(C\left(\mathbb{E}_{1}\right) \times\right.$ $\left.C\left(\mathbb{E}_{1}\right), J_{\tau_{1}} \times J_{\tau_{1}^{\prime}}\right) \simeq\left(C\left(\mathbb{E}_{2}\right) \times C\left(\mathbb{E}_{2}\right), J_{\tau_{2}} \times J_{\tau_{2}^{\prime}}\right)$, then $\theta\left(\tau_{1}\right)=\theta\left(\tau_{2}\right)$. 
Proof. By the hypothesis we have $\left(C\left(\mathbb{E}_{1}\right) \times C\left(\mathbb{E}_{1}\right), J_{\tau_{1}} \times J_{\tau_{1}}\right) \simeq\left(C\left(\mathbb{E}_{2}\right) \times\right.$ $\left.C\left(\mathbb{E}_{2}\right), J_{\tau_{2}} \times J_{\tau_{2}}\right)$ or $\left(C\left(\mathbb{E}_{1}\right) \times C\left(\mathbb{E}_{1}\right), J_{\tau_{1}} \times J_{\text {id }}\right) \simeq\left(C\left(\mathbb{E}_{2}\right) \times C\left(\mathbb{E}_{2}\right), J_{\tau_{2}} \times J_{\text {id }}\right)$. In the first case by $(7.12)$, we have $\left(C\left(\mathbb{E}_{1}\right), J_{\tau_{1}}\right) \simeq\left(C\left(\mathbb{E}_{2}\right), J_{\tau_{2}}\right)$ which implies that $\theta\left(\tau_{1}\right)=\theta\left(\tau_{2}\right)$. So consider the second case. By $(4.8(b)),\left(C\left(\mathbb{E}_{1}\right), J_{\tau_{1}}\right)$ is of orthogonal type and by $(4.8(a)),\left(C\left(\mathbb{E}_{2}\right), J_{\mathrm{id}}\right)$ is of symplectic type, so $\left(C\left(\mathbb{E}_{1}\right), J_{\tau_{1}}\right) \nsucceq\left(C\left(\mathbb{E}_{2}\right), J_{\mathrm{id}}\right)$. Again by $(7.12)$, we have $\left(C\left(\mathbb{E}_{1}\right), J_{\tau_{1}}\right) \simeq\left(C\left(\mathbb{E}_{2}\right), J_{\tau_{2}}\right)$, in particular $\theta\left(\tau_{1}\right)=\theta\left(\tau_{2}\right)$.

Proposition 7.17. For $n \geqslant 2$, let $(V, q)$ be a $2 n$-dimensional quadratic space over a field $F$ of characteristic 2 and let $\tau$ be an involution in $O(V, q)$. Suppose that $\Delta(q)$ is trivial and $\operatorname{dim} \operatorname{Fix}(V, \tau)=\frac{1}{2} \operatorname{dim} V$.

(i) If $\tau$ is of reflectional kind and $\left(C_{0}(V), J_{\tau}\right) \simeq\left(C\left(V^{\prime}\right) \times C\left(V^{\prime}\right), J_{\tau^{\prime}} \times J_{\tau^{\prime}}\right)$ is the isomorphism in $(7.11(\mathrm{a}))$, where $\left(V^{\prime}, q^{\prime}\right)$ is a $(2 n-2)$-dimensional quadratic space over $F$ and $\tau^{\prime}$ is an involution in $O\left(V^{\prime}, q^{\prime}\right)$ which is an orthogonal sum of reflections (in particular by $(4.7),\left(C\left(V^{\prime}\right), J_{\tau^{\prime}}\right)$ is of orthogonal type), then

(a) If $n=2$ then $\operatorname{disc} J_{\tau^{\prime}}=\theta(\tau)$.

(b) If $n \geqslant 3$ then $\operatorname{disc} J_{\tau^{\prime}}=1 \in F^{\times} / F^{\times 2}$.

(ii) If $\tau$ is of interchanging kind and $\left(C_{0}(V), J_{\tau}\right) \simeq\left(C\left(V^{\prime}\right) \times C\left(V^{\prime \prime}\right), J_{\tau^{\prime}} \times\right.$ $\left.J_{\text {id }}\right)$ is the isomorphism of $(7.11(b))$, where $\left(V^{\prime}, q^{\prime}\right)$ and $\left(V^{\prime \prime}, q^{\prime \prime}\right)$ are $(2 n-2)$ dimensional quadratic space over $F$ and $\tau^{\prime}$ is an involution in $O\left(V^{\prime}, q^{\prime}\right)$ which is an orthogonal sum of reflections (in particular by $(4.7),\left(C\left(V^{\prime}\right), J_{\tau^{\prime}}\right)$ is of orthogonal type), then $\operatorname{disc} J_{\tau^{\prime}}=1 \in F^{\times} / F^{\times 2}$.

Proof. (i) (a) Note that by $(4.11(a))$, disc $J_{\tau^{\prime}}=\theta\left(\tau^{\prime}\right)$ and by $(7.16), \theta\left(\tau^{\prime}\right)$ is independent of the choice of $V^{\prime}$. As $\operatorname{dim} \operatorname{Fix}(V, \tau)=\frac{1}{2} \operatorname{dim} V,(V, \tau)$ has trivial maximal fixed orthogonal summand. Since $\tau$ is of reflectional kind, $(V, \tau)$ has a Wiitala decomposition $V=\mathbb{E}_{1} \perp \mathbb{E}_{2}$ where $\tau_{i}=\left.\tau\right|_{\mathbb{E}_{i}}$ is a reflection along some anisotropic vector $u_{i} \in \mathbb{E}_{i}, i=1,2$. By (7.8), we can write $\left(C_{0}(V), J_{\tau}\right) \simeq$ $\left(C\left(\mathbb{E}_{2}, a \cdot q\right) \times C\left(\mathbb{E}_{2}, a \cdot q\right), J_{\tau_{2}} \times J_{\tau_{2}}\right)$ where $a=q\left(u_{1}\right)$. By $(4.11(a))$, disc $J_{\tau_{2}}=$ $\theta\left(\tau_{2}\right)=a q\left(u_{2}\right) F^{\times 2}=q\left(u_{1}\right) q\left(u_{2}\right) F^{\times 2}=\theta(\tau)$.

(b) We have $\operatorname{dim} V^{\prime} \geqslant 4$, so by $(4.11(b))$, disc $J_{\tau^{\prime}}$ is trivial.

(ii) If $n=2$, by (7.10), we can choose $\left(V^{\prime}, q^{\prime}\right)=\left(V^{\prime \prime}, q^{\prime \prime}\right)=(\mathbb{H}, \varphi)$ where $(\mathbb{H}, \varphi)$ is a hyperbolic plane over $F$ and $\tau^{\prime}=\tau_{u}$ where $\varphi(u)=1$. Also by $(7.16), \theta\left(\tau^{\prime}\right)$ is independent of the choice of $\mathbb{E}$. So by (4.11), disc $J_{\tau^{\prime}}=\varphi(u) F^{\times 2}=1 \in F / F^{\times 2}$. If $n \geqslant 3$, we have $\operatorname{dim} V^{\prime} \geqslant 4$, so by $(4.11(b))$, $\operatorname{disc} J_{\tau^{\prime}}$ is trivial.

\section{A Appendix}

Definition A.1. Let $(V, q)$ be a quadratic space over a field $F$ of characteristic 2 and let $A$ be an $F$-algebra. A bilinear map $\psi: V \times V \rightarrow A$ is called an even Clifford map if for every $x, y, z \in V$ we have:

(a) $\psi(x, y) \psi(y, z)=q(y) \cdot \psi(x, z)$.

(b) $\psi(x, x)=q(x) \cdot 1_{A}$.

Remark A.2. Let $(V, q, B)$ be a quadratic space over a field $F$ of characteristic 2 and let $A$ be an $F$-algebra. Let $\psi: V \times V \rightarrow A$ be an even Clifford map and $u, v \in V$. Then by computing $\psi(u+v, u+v)$, we have $\psi(u, v)+\psi(v, u)=$ $B(u, v) \cdot 1_{A}$. 
Lemma A.3. Let $(V, q, B)$ be a quadratic space over a field $F$ of characteristic 2 and let $A$ be an $F$-algebra. Let $\psi: V \times V \rightarrow A$ be an even Clifford map and $u, v, w, x \in V$. Then $\psi(u, v) \psi(w, x)=\psi(u, w) \psi(v, x)+B(v, w) \cdot \psi(u, x)$.

Proof. Using (a) in (A.1) and the bilinearity of $\psi$, we have

$q(v+w) \cdot \psi(u, x)=(q(v+w)+B(v, w)) \cdot \psi(u, x)+\psi(u, v) \psi(w, x)+\psi(u, w) \psi(v, x)$.

So $\psi(u, v) \psi(w, x)=\psi(u, w) \psi(v, x)+B(v, w) \cdot \psi(u, x)$.

Corollary A.4. Let $(V, q, B)$ be a quadratic space over a field $F$ of characteristic 2 and let $A$ be an $F$-algebra. Let $\psi: V \times V \rightarrow A$ be an even Clifford map and $u, v \in V$. Then $\psi(u, v) \psi(u, v)=q(u) q(v) \cdot 1_{A}+B(u, v) \cdot \psi(u, v)$.

Theorem A.5. Let $(V, q)$ be a quadratic space over a field $F$ of characteristic 2. There exists an even Clifford map $j$ from $V \times V$ to $C_{0}(V)$ which satisfies the following conditions:

(a) As an $F$-algebra $C_{0}(V)$ is generated by $1_{C_{0}(V)}$ and $\{j(x, y) \mid x, y \in V\}$.

(b) For every even Clifford map $\psi: V \times V \rightarrow A$, there exists a unique $F$ algebra homomorphism $\Psi: C_{0}(V) \rightarrow A$ such that $\psi=\Psi \circ j$, i.e., the following diagram commutes:

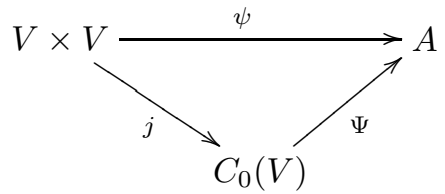

Proof. Define $j: V \times V \rightarrow C_{0}(V)$ via $j(u, v)=u v$. Then $j$ is clearly an even Clifford map. Let $\psi: V \times V \rightarrow A$ be an even Clifford map. Let $u_{1} \in V$ be an anisotropic vector and set $a=q\left(u_{1}\right)$. Extend $\left\{u_{1}\right\}$ to a symplectic basis $\left\{u_{1}, v_{1}, \cdots, u_{n}, v_{n}\right\}$ of $V$ and set $z=u_{1} v_{1}+\cdots u_{n} v_{n}$. By (4.12), we have $z^{2}=z+\delta \cdot 1$ where $\delta=\sum_{i=1}^{n} q\left(u_{i}\right) q\left(v_{i}\right) \in F$ is a representative of the class $\Delta(q) \in F / \wp(F)$. By $[4,(13.12)]$, the map $g: C_{0}(V) \rightarrow C(W, a \cdot q) \otimes(F+F z)$ induced by $g\left(u_{1} w\right)=w \otimes 1$ and $g(z)=1 \otimes z$ is an isomorphism, where $W$ is the subspace of $V$ generated by $u_{2}, v_{2}, \cdots, u_{n}, v_{n}$. Define the linear map $f_{1}: W \rightarrow A$ via $f_{1}(w)=\psi\left(u_{1}, w\right)$. By (A.4), for every $w \in W$ we have $f_{1}(w)^{2}=\psi\left(u_{1}, w\right) \psi\left(u_{1}, w\right)=q\left(u_{1}\right) q(w) \cdot 1_{A}+B\left(u_{1}, w\right) \cdot \psi\left(u_{1}, w\right)=a \cdot q(w)$. So the map $f_{1}$ is compatible with $a \cdot q$ and it can be extended to an $F$-algebra homomorphism $\bar{f}_{1}: C(W, a \cdot q) \rightarrow A$. Now define the map $f_{2}: F+F z \rightarrow A$ via $f_{2}(\alpha+\beta z)=\alpha+\beta\left(\psi\left(u_{1}, v_{1}\right)+\cdots+\psi\left(u_{n}, v_{n}\right)\right)$. By (A.4), we have $\psi\left(u_{i}, v_{i}\right)^{2}=$ $q\left(u_{i}\right) q\left(v_{i}\right) \cdot 1_{A}+\psi\left(u_{i}, v_{i}\right)$. Also for every $i \neq j$, by (A.2) and (A.3), we have

$\psi\left(u_{i}, v_{i}\right) \psi\left(u_{j}, v_{j}\right)=\psi\left(u_{i}, u_{j}\right) \psi\left(v_{i}, v_{j}\right)=\psi\left(u_{j}, u_{i}\right) \psi\left(v_{j}, v_{i}\right)=\psi\left(u_{j}, v_{j}\right) \psi\left(u_{i}, v_{i}\right)$

So

$$
\begin{aligned}
f_{2}(z)^{2} & =\left(\psi\left(u_{1}, v_{1}\right)+\cdots+\psi\left(u_{n}, v_{n}\right)\right)^{2}=\psi\left(u_{1}, v_{1}\right)^{2}+\cdots+\psi\left(u_{n}, v_{n}\right)^{2} \\
& =q\left(u_{1}\right) q\left(v_{1}\right) \cdot 1_{A}+\psi\left(u_{1}, v_{1}\right)+\cdots+q\left(u_{n}\right) q\left(v_{n}\right) \cdot 1_{A}+\psi\left(u_{n}, v_{n}\right) \\
& =\delta \cdot 1_{A}+\psi\left(u_{1}, v_{1}\right)+\cdots+\psi\left(u_{n}, v_{n}\right)=f_{2}(\delta+z)=f_{2}\left(z^{2}\right) .
\end{aligned}
$$


Therefore, $f_{2}\left(\left(\alpha_{1}+\beta_{1} z\right)\left(\alpha_{2}+\beta_{2} z\right)\right)=f_{2}\left(\alpha_{1}+\beta_{1} z\right) f_{2}\left(\alpha_{2}+\beta_{2} z\right)$ for every $\alpha_{1}, \beta_{1}, \alpha_{2}, \beta_{2} \in F$ and $f_{2}$ is an $F$-algebra homomorphism.

We claim that the image of $\bar{f}_{1}$ commutes with that of $f_{2}$. We must show that for every $w \in W$ :

$\psi\left(u_{1}, w\right)\left(\psi\left(u_{1}, v_{1}\right)+\cdots+\psi\left(u_{n}, v_{n}\right)\right)=\left(\psi\left(u_{1}, v_{1}\right)+\cdots+\psi\left(u_{n}, v_{n}\right)\right) \psi\left(u_{1}, w\right)$.

It is enough to check this for $w=u_{i}$ and $w=v_{i}, i=1, \cdots, n$. If $i=1,(6)$ is clearly true. So fix an index $i$ with $2 \leqslant i \leqslant n$. If $j \neq 1$ and $j \neq i$, by (A.2) and (A.3), we have $\psi\left(u_{1}, u_{i}\right) \psi\left(u_{j}, v_{j}\right)=\psi\left(u_{j}, v_{j}\right) \psi\left(u_{1}, u_{i}\right)$. If $j=1$ or $j=i$, we have

$$
\begin{aligned}
\psi\left(u_{1}, u_{i}\right) \psi\left(u_{1}, v_{1}\right) & +\psi\left(u_{1}, u_{i}\right) \psi\left(u_{i}, v_{i}\right) \\
& =\psi\left(u_{1}, u_{1}\right) \psi\left(u_{i}, v_{1}\right)+B\left(u_{i}, u_{1}\right) \cdot \psi\left(u_{1}, v_{1}\right)+q\left(u_{i}\right) \cdot \psi\left(u_{1}, v_{i}\right) \\
& =q\left(u_{1}\right) \cdot \psi\left(u_{i}, v_{1}\right)+q\left(u_{i}\right) \cdot \psi\left(u_{1}, v_{i}\right) .
\end{aligned}
$$

Similarly

$$
\psi\left(u_{1}, v_{1}\right) \psi\left(u_{1}, u_{i}\right)+\psi\left(u_{i}, v_{i}\right) \psi\left(u_{1}, u_{i}\right)=q\left(u_{1}\right) \cdot \psi\left(u_{i}, v_{1}\right)+q\left(u_{i}\right) \cdot \psi\left(u_{1}, v_{i}\right) .
$$

So $\psi\left(u_{1}, u_{i}\right) \psi\left(u_{1}, v_{1}\right)+\psi\left(u_{1}, u_{i}\right) \psi\left(u_{i}, v_{i}\right)=\psi\left(u_{1}, v_{1}\right) \psi\left(u_{1}, u_{i}\right)+\psi\left(u_{i}, v_{i}\right) \psi\left(u_{1}, u_{i}\right)$ and the equality (6) is achieved for $w=u_{i}$. Similarly (6) is true for $w=v_{i}$ and the claim is proved. By [2, Ch. 5, Thm. 2] one can consider the $F$-algebra homomorphism $f=\bar{f}_{1} \otimes f_{2}: C(W, a \cdot q) \otimes(F+F z) \rightarrow A$.

Set $\Psi=f \circ g$. We claim that $\psi=\Psi \circ j$. For every $v \in V$ we can write $v=\alpha u_{1}+\beta v_{1}+w$, where $\alpha, \beta \in F$ and $w \in W$. Since $j\left(v, v^{\prime}\right)=v v^{\prime}$ for every $v, v^{\prime} \in V$, it is enough to show that $\psi\left(u_{1}, u_{1}\right)=\Psi\left(u_{1} u_{1}\right), \psi\left(v_{1}, v_{1}\right)=\Psi\left(v_{1} v_{1}\right)$, $\psi\left(u_{1}, v_{1}\right)=\Psi\left(u_{1} v_{1}\right), \psi\left(u_{1}, w\right)=\Psi\left(u_{1} w\right), \psi\left(v_{1}, w\right)=\Psi\left(v_{1} w\right)$ and $\psi\left(w_{1}, w_{2}\right)=$ $\Psi\left(w_{1} w_{2}\right)$ for every $w, w_{1}, w_{2} \in W$. The first two relations follow from the $F$ linearity of $\Psi$ and the property (b) of (A.1). For every $w \in W$ we have

$$
\Psi\left(u_{1} w\right)=f \circ g\left(u_{1} w\right)=\bar{f}_{1}(w)=\psi\left(u_{1}, w\right)
$$

Similarly

$$
\begin{aligned}
\Psi\left(w_{1} w_{2}\right) & =a^{-1} \cdot \Psi\left(u_{1} w_{1} u_{1} w_{2}\right)=a^{-1} \cdot(f \circ g)\left(u_{1} w_{1}\right)(f \circ g)\left(u_{1} w_{2}\right) \\
& =a^{-1} \cdot \bar{f}_{1}\left(w_{1}\right) \bar{f}_{1}\left(w_{2}\right)=a^{-1} \cdot \psi\left(u_{1}, w_{1}\right) \psi\left(u_{1}, w_{2}\right) \\
& =a^{-1} \cdot \psi\left(w_{1}, u_{1}\right) \psi\left(u_{1}, w_{2}\right)=a^{-1} q\left(u_{1}\right) \cdot \psi\left(w_{1}, w_{2}\right)=\psi\left(w_{1}, w_{2}\right) .
\end{aligned}
$$

The relations $\Psi\left(u_{1} v_{1}\right)=\psi\left(u_{1}, v_{1}\right)$ and $\Psi\left(w v_{1}\right)=\psi\left(w, v_{1}\right)$ are obtained with the same arguments. For the last one, we use $w v_{1}=v_{1} w=a^{-1}\left(w u_{1} z+w u_{1} u_{2} v_{2}+\right.$ $\left.\cdots+w u_{1} u_{n} v_{n}\right)$.

Finally, the uniqueness of $\Psi$ follows from the facts that as an $F$-algebra, $C_{0}(V)$ is generated by $1_{C_{0}(V)}$ and the set $\{j(u, v) \mid u, v \in V\}$, and the relation $\Psi(u v)=\Psi \circ j(u, v)=\psi(u, v)$.

\section{References}

[1] K. J. Becher, A proof of the Pfister factor conjecture. Invent. Math. 173 (2008), no. 1, 1-6. 
[2] P. K. Draxl, Skew fields. London Mathematical Society Lecture Note Series, 81. Cambridge University Press, Cambridge, 1983.

[3] R. S. Garibaldi, Clifford algebras of hyperbolic involutions. Math. Z. 236 (2001), no. 2, 321-349.

[4] L. C. Grove, Classical Groups and Geometric Algebra. Graduate Studies in Mathematics, 39. American Mathematical Society, Providence, RI, 2002 .

[5] N. Karpenko, A. Quéguiner, A criterion of decomposability for degree 4 algebras with unitary involution. J. Pure Appl. Algebra 147 (2000), no. 3, 303-309.

[6] M.-A. Knus, A. S. Merkurjev, M. Rost, J.-P. Tignol, The Book of Involutions. American Mathematical Society Colloquium Publications, 44. American Mathematical Society, Providence, RI, 1998.

[7] M.-A. Knus, R. Parimala, R. Sridharan, Involutions on rank 16 central simple algebras. J. Indian Math. Soc. (N.S.) 57 (1991), no. 1-4, 143-151.

[8] D. W. Lewis, Periodicity of Clifford algebras and exact octagons of Witt groups. Math. Proc. Cambridge Philos. Soc. 98 (1985), no. 2, 263-269.

[9] M. G. Mahmoudi, Orthogonal symmetries and Clifford algebras. Proc. Indian Acad. Sci. Math. Sci. 120 (2010), no. 5, 535-561.

[10] P. Mammone, J.-P. Tignol, A. Wadsworth, Fields of characteristic 2 with prescribed $u$-invariants. Math. Ann. 290 (1991), no. 1, 109-128.

[11] A. Masquelein, A. Quéguiner-Mathieu, J.-P. Tignol, Quadratic forms of dimension 8 with trivial discriminant and Clifford algebra of index 4 . Arch. Math. (Basel) 93 (2009), no. 2, 129-138.

[12] A. Pfister, Quadratic forms with applications to algebraic geometry and topology. London Mathematical Society Lecture Note Series, 217. Cambridge University Press, Cambridge, 1995.

[13] A. Quéguiner-Mathieu, J.-P. Tignol, Algebras with involution that become hyperbolic over the function field of a conic. Israel J. Math. 180 (2010), 317-344.

[14] W. Scharlau, Quadratic and Hermitian Forms. Grundlehren der Mathematischen Wissenschaften, 270. Springer-Verlag, Berlin, 1985.

[15] D. B. Shapiro, Compositions of quadratic forms. de Gruyter Expositions in Mathematics, 33. Walter de Gruyter \& Co., Berlin, 2000.

[16] A. S. Sivatski, Applications of Clifford algebras to involutions and quadratic forms. Comm. Algebra 33 (2005), no. 3, 937-951.

[17] S. A. Wiitala, Factorization of involutions in characteristic two orthogonal groups: an application of the Jordan form to group theory. Linear Algebra and Appl., 21 (1978), no. 1, 59-64.

M. G. MAHMOUd, mmahmoudi@sharif.ir, A.-H. NOKHODKAR, anokhodkar@yahoo.com Department of Mathematical Sciences, Sharif University of Technology, P. O. Box 11155-9415, TEHran, Iran. 